\title{
Observational Signatures of Cosmic-Ray Interactions in Molecular Clouds
}

\author{
Ellis R. Owen ${ }^{1,2}$ (1) Alvina Y. L. On ${ }^{1,2,3}$ (1) , Shih-Ping Lai ${ }^{1}$ (i), and Kinwah $\mathrm{Wu}^{3,4}$ (1) \\ ${ }^{1}$ Institute of Astronomy, Department of Physics, National Tsing Hua University, Hsinchu, Taiwan (ROC); erowen@gapp.nthu.edu.tw \\ ${ }^{2}$ Center for Informatics and Computation in Astronomy, National Tsing Hua University, Hsinchu, Taiwan (ROC) \\ ${ }^{3}$ Mullard Space Science Laboratory, University College London, Holmbury St. Mary, Dorking, Surrey, RH5 6NT, UK \\ ${ }^{4}$ Research center for Astronomy, Astrophysics and Astrophotonics, Macquarie University, Sydney, NSW 2109, Australia \\ Received 2020 November 15; revised 2021 March 2; accepted 2021 March 10; published 2021 May 24
}

\begin{abstract}
We investigate ionization and heating of gas in the dense, shielded clumps/cores of molecular clouds bathed by an influx of energetic, charged cosmic rays (CRs). These molecular clouds have complex structures, with substantial variation in their physical properties over a wide range of length scales. The propagation and distribution of CRs is thus regulated accordingly, in particular, by the magnetic fields threaded through the clouds and into the dense regions within. We have found that a specific heating rate reaching $10^{-26} \mathrm{erg} \mathrm{cm}^{-3} \mathrm{~s}^{-1}$ can be sustained in the dense clumps/cores for Galactic environments, and this rate increases with CR energy density. The propagation of CRs and heating rates in some star-forming filaments identified in IC 5146 are calculated, with the CR diffusion coefficients in these structures determined from magnetic field fluctuations inferred from optical and near-infrared polarizations of starlight, which is presumably a magnetic field tracer. Our calculations indicate that CR heating can vary by nearly three orders of magnitude between different filaments within a cloud due to different levels of $\mathrm{CR}$ penetration. The $\mathrm{CR}$ ionization rate among these filaments is similar. The equilibrium temperature that could be maintained by $\mathrm{CR}$ heating alone is of order $1 \mathrm{~K}$ in a Galactic environment, but this value would be higher in strongly star-forming environments, thus causing an increase in the Jeans mass of their molecular clouds.
\end{abstract}

Unified Astronomy Thesaurus concepts: Interstellar clouds (834); Cosmic rays (329); Galactic cosmic rays (567); Interstellar magnetic fields (845); Star formation (1569)

\section{Introduction}

The dense cores of molecular clouds (MCs) within our Galaxy are expected to be shielded from much of the ionizing (particularly ultraviolet) interstellar radiation by dust and molecular hydrogen (Draine 2011). However, observations have revealed sustained ionization rates of up to $\zeta^{\mathrm{H}}=10^{-17}-10^{-15} \mathrm{~s}^{-1}$ in dense cores (Caselli et al. 1998; van der Tak \& van Dishoeck 2000; Doty et al. 2002), compared with $\zeta^{\mathrm{H}}=10^{-16} \mathrm{~s}^{-1}$ in diffuse interstellar clouds (Black et al. 1978; van Dishoeck \& Black 1986; Federman et al. 1996). The cause of this ionization is widely attributed to cosmic rays (CRs; Goldsmith \& Langer 1978; Goldsmith 2001; Lequeux 2005; Draine 2011), which would also act to regulate the temperature (e.g., Spitzer \& Tomasko 1968) and chemical evolution (e.g., Desch et al. 2004; Dalgarno 2006; Indriolo et al. 2015; Bisbas et al. 2017; Albertsson et al. 2018; Padovani et al. 2018; Gaches et al. 2019) of MCs. In this paper, we model the propagation of CRs in MC environments, accounting for their injection/absorption via hadronic interactions and ionizations in a self-consistent manner. We also demonstrate how this model can be applied to polarization observations of MCs and their cores, from which CR propagation parameters and ionization/heating patterns can be determined.

CRs are energetic, charged particles. They are able to penetrate into MCs, causing ionization in dense MC cores which are inaccessible to interstellar ionizing radiation. CR protons in the $\mathrm{MeV}-\mathrm{GeV}$ energy range and $\mathrm{CR}$ electrons in the $10 \mathrm{keV}-10 \mathrm{MeV}$ range are believed to contribute to the bulk of this ionization (e.g., Spitzer \& Tomasko 1968; Padovani \& Galli 2011; Yamamoto 2017). Limits on CR ionization rates in interstellar clouds were first calculated by Hayakawa et al. (1961) to be up to $10^{-15} \mathrm{~s}^{-1}$. Later, Spitzer \& Tomasko (1968) indicated a range from $\zeta^{\mathrm{H}} \approx 6.8 \times 10^{-18} \mathrm{~s}^{-1}$ (when invoking a CR proton spectrum declining below $50 \mathrm{MeV}$ ), to values as high as $\zeta^{\mathrm{H}} \approx 1.2 \times 10^{-15} \mathrm{~s}^{-1}$ (when accounting for $\mathrm{MeV}$ protons injected by supernova (SN) events). The upper end of this range had been disputed for some time, particularly by researchers inferring $\zeta^{\mathrm{H}}$ from the chemical balances of species influenced by CR ionization; however, later studies showed CR ionization rates to be consistent with the lower end of this range (e.g., Glassgold \& Langer 1974), with a consensus now having largely been reached, that the rate is around $10^{-16} \mathrm{~s}^{-1}$ for diffuse interstellar cloud environments (see, e.g., Black et al. 1978; Hartquist et al. 1978; van Dishoeck \& Black 1986; Federman et al. 1996; Geballe et al. 1999, 2007; Indriolo et al. 2007; Indriolo 2012; Indriolo \& McCall 2012; Padovani et al. 2009, hereafter P09, 2020; Draine 2011; Indriolo 2013 for overviews). CRs with energies above a $\mathrm{GeV}$ can also play a role (Bykov et al. 2020). These are associated with star-forming activities, which yield massive stellar end products, e.g., SN remnants (see Blasi 2011 for a discussion). Such environments can accelerate particles to relativistic energies through, e.g., Fermi (1949) acceleration in diffusive shocks (Axford et al. 1977; Krymskii 1977; Bell 1978a, 1978b; Blandford \& Ostriker 1978). At these high energies, pion-producing $\left(\pi^{0}, \pi^{ \pm}\right)$pair-production (pp) interactions between CR protons and the dense MC gas can arise (see Kafexhiu et al. 2014; Owen et al. 2018). The decay of $\pi^{ \pm}$yields secondary $\mathrm{MeV} \mathrm{CR}$ electrons, and these are deposited locally (i.e., within the dense cloud). ${ }^{5}$ These higher-energy CRs can also engage with the ambient magnetic field and can drive a gas heating rate via Alfvén wave excitation (Wentzel 1971; Wiener et al. 2013b).

The ionization level of a dense cloud governs the degree to which it is coupled to its ambient magnetic field. This, in turn,

\footnotetext{
5 We estimate that the contribution of these $\mathrm{MeV} \mathrm{CR}$ electrons from pion decays to the ionization rate is negligible. See Section 4.1.4 for a further discussion.
} 
regulates its stability against fragmentation and/or gravitational collapse (Mestel \& Spitzer 1956; Price \& Bate 2008) and influences its subsequent star-forming activities. The propagation of charged CRs is governed by the local magnetic field, which can develop a very complicated structure (Padovani et al. 2013) with elevated field strengths arising as it co-evolves with its host cloud (Crutcher 2012). In these strengthened complex magnetic fields, CRs may be focused by the field morphology to cause a convergence in their diffusive propagation. On the other hand, CRs may also be reflected/deflected as their pitch angles increase due to the stronger magnetic field. These antagonistic processes generally act concurrently (Cesarsky \& Volk 1978; Ko 1992; Chandran 2000; Desch et al. 2004; Padoan \& Scalo 2005), and it has recently been argued that the mirroring/deflection effect always dominates over focusing such that the CR flux reaching the densest magnetized core regions is slightly reduced overall by a factor of 2 or 3 (Padovani \& Galli 2011; Padovani et al. 2013; Silsbee et al. 2018) compared to the exterior cloud environment (if other effects, e.g., energy losses and absorption interactions, are ignored; at $\mathrm{MeV}$ energies, for example, energy losses would dominate and could prevent CR propagation into the densest core regions entirely (see Chernyshov et al. 2018).

This study investigates the ionization and heating of MCs in the presence of CR irradiation. We consider a model for the propagation of CRs in specified MC magnetic field and density structures, also accounting for secondary electrons arising through hadronic interactions of primary CR protons. We determine gas temperatures and molecular abundances through calculating the chemical balances of species, including $\mathrm{HCO}^{+}$, $\mathrm{H}_{3}^{+}, \mathrm{OH}^{+}$, and $\mathrm{H}_{2} \mathrm{O}^{+}$, and determine temperature profiles and chemical balances in model MCs when irradiated by CR fluxes typical of the Galactic interstellar environment. We also predict the corresponding outcome if the irradiating CR intensity changes. We then apply our model to the interstellar cloud IC 5146 , a complex known to host a broad variety of environments for which a plethora of polarization measurements are available (Wang et al. 2017, 2020).

The paper is organized as follows. Section 2.1 outlines the characteristics of MC environments based on studies of the Milky Way and nearby galaxies. A model is introduced to describe the density and magnetic structures of MCs, identifying the dominant components engaging with CRs. Sections 2.2-2.4 present the relevant $\mathrm{CR}$ physics - $\mathrm{CR}$ propagation in $\mathrm{MC}$ environments and the interaction channels for both hadronic and leptonic CRs. Section 3 details the analysis of magnetic field structures in MC environments and demonstrates how the propagation parameters of CRs can be determined from this. Section 4 presents our results, showing $\mathrm{CR}$ heating and ionization rates in a model MC and in the dense filamentary structures of IC 5146. We provide a brief summary and conclusions in Section 5 .

\section{CRs in MCs}

\section{1. $M C s$}

The interstellar medium is multiphase, with cold, dense neutral MCs intermingled with hot tenuous gases in pressure equilibrium. The clouds condense from the hot interstellar medium (ISM) gas as it cools and collapses under gravity, reaching typical densities of around $10^{2} \mathrm{~cm}^{-3}$, temperatures of around $10 \mathrm{~K}$, and sizes of a few to a few tens of parsecs. These are permeated by magnetic fields of a few microguass, presumably having been swept in from the ISM by the collapsing material to create a "pinching" effect in the field orientation. The resulting structure of the magnetic field vectors has been observed to resemble an "hourglass" (Girart et al. 2006; Rao et al. 2009; Tang et al. 2009), where field lines are drawn closer together in regions of higher cloud density. This produces a much stronger magnetic field within the MC compared to the surrounding ISM (e.g., Crutcher 1999; Basu 2000; Basu et al. 2009). Further gravitational collapse within MCs to form clumps/filaments and cores is mediated by pressure support-the source of this being either the magnetic fields (Mouschovias 1991; Mouschovias \& Ciolek 1999) or turbulence (Padoan \& Nordlund 1999; Mac Low \& Klessen 2004; Zhang \& Li 2017; Coudé et al. 2019), with recent studies suggesting that both could play important roles (Kudoh \& Basu 2008; Vázquez-Semadeni et al. 2011; Seifried \& Walch 2015; Federrath 2016; Planck Collaboration et al. 2016). This leads to a hierarchical substructure, wherein clouds host clumps of size $0.3-3$ pc (or filaments of comparable width, e.g., see Arzoumanian et al. 2011) and density of $10^{3}-10^{4} \mathrm{~cm}^{-3}$. Moreover, cores on scales of 0.03-0.2 pc may develop within these clumps/filaments. Cores have higher densities of around $10^{4}-10^{5} \mathrm{~cm}^{-3}$, with some cases even reaching $10^{6}-10^{7} \mathrm{~cm}^{-3}$ (see Bergin \& Tafalla 2007 for a review). Note that different distinctions between the components are also proposed in the literature and are equally valid (e.g., Myers 1995). This is because an exact description of the continuous substructure of MCs cannot fully be captured by a simple hierarchy of just a few distinct elements (Rodríguez 2005).

Polarization observations have indicated complex structures in the magnetic fields toward dense cores (see, e.g., Hull et al. 2017). This indicates that turbulence may be more important than magnetic fields in governing their internal dynamics. However, there are some massive cores with ordered magnetic fields on $0.1-0.01 \mathrm{pc}$ scales, which are mostly parallel or perpendicular to ordered fields on larger scales (Zhang et al. 2014). These are generally also found to be either parallel or perpendicular to the observed orientation of their host cloud ( $\mathrm{Li}$ et al. 2015), suggesting that they are of dynamical importance in regulating the initial cloud collapse/fragmentation processes (see also Koch et al. 2014). Li et al. (2009) arrived at a similar conclusion when comparing magnetic fields on 1 and $100 \mathrm{pc}$ scales, but Zhang et al. (2019) found that this correlation does not extend to sub-parsec $(0.1-0.01 \mathrm{pc})$ scales where turbulence may have a more important role, possibly concentrated by gravitational collapse to create slightly super-Alfvénic cores in otherwise sub-Alfvénic host clouds (see Ching et al. 2017; Tang et al. 2019). Being of higher density and smaller size, the cores themselves are observed to sustain typical magnetic field strengths of around $10-15 \mu \mathrm{G}$ (Crutcher 1999), ${ }^{6}$ while the mean line of sight and total magnetic field strengths in the intercore regions of MCs have been observed to be 7.4 and $14.8 \mu \mathrm{G}$, respectively, via the Zeeman effect (Thompson et al. 2019).

We consider a three-zone model for the structure of an MC and core in which we compute the interactions and propagation of energetic particles. The model, as illustrated in Figure 1, has an external ISM region outside of the cloud, Zone 1, where CRs propagate diffusively, and the medium is almost fully ionized $x_{i} \approx 1$. From Zones $2-3$, the magnetic field pinching effect resulting from the cloud's evolution would begin to influence the CR propagation, while the increased density/neutral

\footnotetext{
6 Note that observations show substantial spread in this value, which seems to be sensitive to the type of core observed (Crutcher et al. 2010).
} 


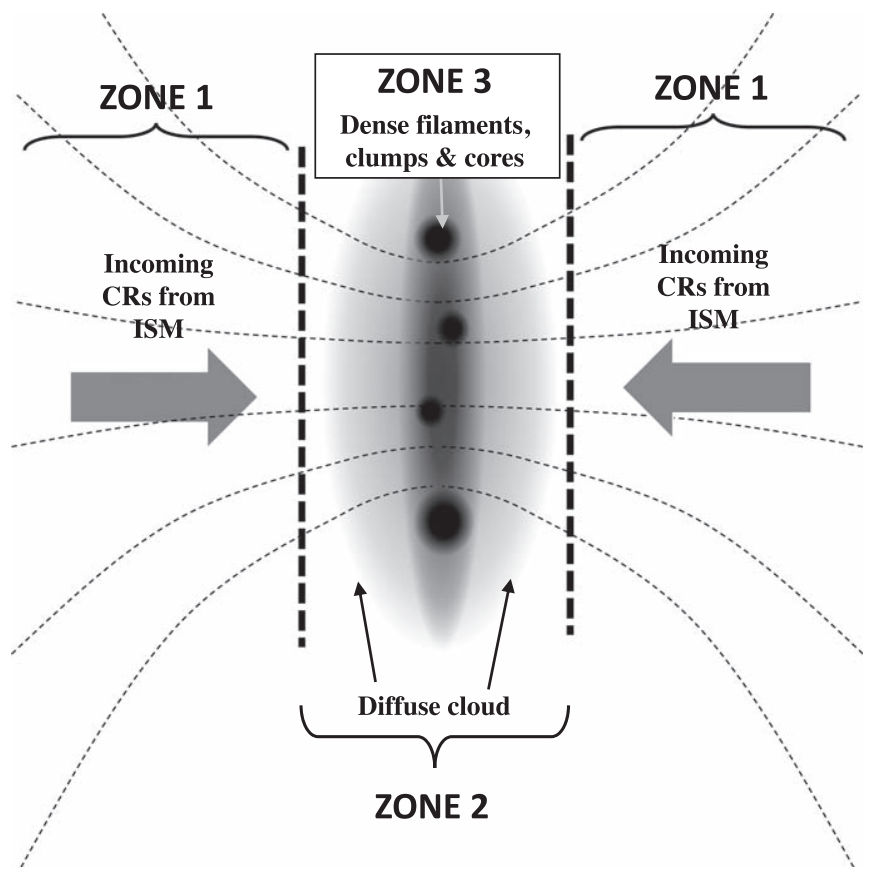

Figure 1. Schematic of the different $\mathrm{CR}$ propagation regions within the density and magnetic field profiles of an idealized MC, characterizing observed features (e.g., Rao et al. 2009). Zone 1 is the ISM region $\left(x_{i} \approx 1\right)$. In Zone 2 , the magnetic field pinching effect and increased density/neutral fraction of the cloud begins to affect the propagation of CRs, which becomes decreasingly diffusive as the higher neutral fraction damps the Alfvén waves (Kulsrud \& Pearce 1969; Zweibel \& Shull 1982). Zone 3 is the dense filament/clump (containing dense cores) of the MC where magnetic fields are the strongest and densities the highest. The gas is almost completely neutral $\left(x_{i} \approx 10^{-7}\right.$ or less, e.g., see Draine 2011) except for the ionization caused by CRs. Magnetic focusing/mirroring effects become important in Zone 3.

fraction would lead it to become decreasingly diffusive (compared to the ISM outside the cloud). As long as the magnetic field orientations and density structure are known at any given location, our approach can be adapted to account for any likely MC structure - not just the hourglass shape indicated by the figure. In this simplified model, we consider only a single core (Zone 3) within the MC, but this can be extended to any number of cores as required. Unless otherwise specified, $n_{\mathrm{H}}$ refers to the number density of all gas within the cloud, whether atomic or molecular hydrogen.

\subsection{Effects of CRs}

\subsubsection{Ionization and Chemical Tracers}

CR ionization rates are studied observationally through the impact they have on the astrochemistry of MCs. Along a line of sight, the column density $N$ of certain species is considered to probe their relative abundances, assuming cospatiality, ${ }^{7}$ and $\zeta^{\mathrm{H}}$ (the total CR ionization rate) can be inferred from these. Of particular interest are species that may be readily observed and are sensitive to $\mathrm{CR}$ ionization: $\mathrm{CO}^{+}, \mathrm{OH}^{+}$, and $\mathrm{C}^{+}$being three such examples that we consider here. Analyzing chemical formation and destruction channels and their rates allows abundances to be modeled in a steady-state chemical system (see Appendix E). Observationally, these would typically be probed using abundance ratios, integrated over a line of sight,

\footnotetext{
This is usually necessary due to limited information about the line-of-sight structure.
}

between two chemically related species. We consider the ratios $N\left(\mathrm{CO}^{+}\right) / N\left(\mathrm{H}_{2}\right), \quad N\left(\mathrm{OH}^{+}\right) / N\left(\mathrm{H}_{2}\right)$, and $N\left(\mathrm{C}^{+}\right) / N(\mathrm{C})$, being closely related species (via reactions P6, P4 of Table E1 and carbon ionization, respectively), by invoking a simplified astrochemical network (see Table E1) for key reaction chains involving $\mathrm{C}, \mathrm{O}$, and molecular/atomic hydrogen as initiated by $\mathrm{CR}$ ionizations, in which a constant temperature is imposed. Our simplified network is strictly valid only in cold dense regions on scales of less than $1 \mathrm{pc}$, where the gas temperature is unlikely to exceed $10 \mathrm{~K}$. However, for this proof-of-concept study, we adopt a binary ionization approximation where CRs either ionize or do not ionize a species in an interaction event. This allows us to neglect excited atomic/molecular states and associated modifications required for the rate coefficients of reactions, therefore relaxing the limit on temperature. We note that recent theoretical work, e.g., Gaches et al. (2019) has shown that adopting such simplified chemical networks, where abundance ratios are determined from time-integrated chemical rate equations, can lead to an underprediction of ion column densities by as much as an order of magnitude. We therefore caution that the abundance ratio results presented in this work should be regarded as approximate, and could vary by up to an order of magnitude.

While $\mathrm{H}_{3}^{+}$and $\mathrm{HCO}^{+}$are conventional probes of $\mathrm{CR}$ ionization rates in certain systems (e.g., van der Tak \& van Dishoeck 2000), the former is hampered by foreground contamination issues in infrared (IR) bands and constrained by the need for a bright IR background source, while the latter is strongly affected by photoionization. As such, we consider alternative species. $\mathrm{OH}^{+}$ has been recommended by some studies as a more direct measure of $\mathrm{CR}$ ionization, relying on fewer interaction steps in its formation (Hollenbach et al. 2012). The strongest $\mathrm{OH}^{+}$transition is at $972 \mathrm{GHz}$, with previous detections using Herschel (e.g., Neufeld et al. 2010). The ionization potential for the formation of $\mathrm{OH}^{+}$is around $13.0 \mathrm{eV}$, so any CR ionization signal could be easily contaminated by photoionization in diffuse, non-shielded cloud regions, and thus is only appropriate as a tracer of $\mathrm{CR}$ ionization in dense, well-shielded clumps and cores. We derive the abundance ratio

$$
\frac{N\left(\mathrm{OH}^{+}\right)}{N\left(\mathrm{H}_{2}\right)} \approx \frac{\left[\left(4 / f_{\mathrm{H}_{2}}\right)+2\right] \zeta^{\mathrm{H}}}{n_{\mathrm{H}}\left(k_{\mathrm{P} 4} f_{\mathrm{H}_{2}}+2 x_{i} k_{\mathrm{T} 1}\right)}
$$

from Appendix E, using Equation (E5) for the $\mathrm{OH}^{+}$balance, assuming that the rate of process $\mathrm{P} 2$ and the $\mathrm{H}_{3}^{+}$dissociative recombination rate are negligible compared to the ionization rate of $\mathrm{H}$ and $\mathrm{H}_{2}$ (which would be appropriate to probe dense cores/clumps). Here, $f_{\mathrm{H}_{2}}$ is the molecular hydrogen fraction (defined as the number of $\mathrm{H}$ nuclei in $\mathrm{H}_{2}$ molecules as a fraction of the total number of $\mathrm{H}$ nuclei per unit volume), and the rate constants $k_{\mathrm{T} 1}$ and $k_{\mathrm{P} 4}$ relate to the processes T1 and $\mathrm{P} 4$ (see Appendix E).

$\mathrm{CO}^{+}$is also a plausible tracer of $\mathrm{CR}$ ionization. It has accessible observable lines at 236.1 (strongest), 235.8, 353.7, and $354.0 \mathrm{GHz}$, which are all within the detection range of the Atacama Large Millimeter/submillimeter Array. The ionization potential in this case is greater than $13.6 \mathrm{eV}$, so $\mathrm{CO}^{+}$would not be susceptible to substantial ionization by interstellar radiation fields, making it a more robust probe of $\mathrm{CR}$ ionization. Even though the strongest $236 \mathrm{GHz}$ line is easily confused with emission from complex molecular species (in particular ${ }^{13} \mathrm{CH}_{3} \mathrm{OH}$ ), several detections have 
now been reported (Latter et al. 1993; Fuente \& MartínPintado 1997; Ceccarelli et al. 1998). We derive the ratio

$$
\frac{N\left(\mathrm{CO}^{+}\right)}{N\left(\mathrm{H}_{2}\right)}=\frac{x_{\mathrm{C}} \zeta^{\mathrm{H}}}{n_{\mathrm{H}}\left(k_{\mathrm{P} 5}+\left(k_{\mathrm{P} 6} f_{\mathrm{H}_{2}} / 2\right)+x_{i} k_{\mathrm{T} 5}\right)},
$$

using the chemical balance for $\mathrm{CO}^{+}$(Equation (E6)), where $x_{\mathrm{C}}$ is the carbon abundance fraction. We further consider the ratio

$$
\frac{N\left(\mathrm{C}^{+}\right)}{N(\mathrm{C})}=\frac{2 \zeta^{\mathrm{H}}}{f_{\mathrm{H}_{2}} n_{\mathrm{H}} x_{\mathrm{OH}} k_{\mathrm{I} 3}}
$$

from the chemical balance of $\mathrm{C}^{+}$from $\mathrm{C}$ ionization in Equation (E3). Here, the rate constants $k_{\mathrm{T} 5}, k_{\mathrm{P} 5}, k_{\mathrm{P} 6}$, and $k_{\mathrm{I} 3}$ are given in Table $\mathrm{E} 1$, and $x_{\mathrm{OH}}$ is the $\mathrm{OH}$ abundance fraction. Although $N\left(\mathrm{C}^{+}\right) / N(\mathrm{C})$ would be contaminated by photoionization in photon-dominated regions and environments that are not well shielded from ionizing interstellar radiation fields, it is a direct measure of the CR ionization rate in denser clumps and cores, which may be complementary to the other line ratios above.

We note that all of these abundance ratios require an estimate for the ambient volume density of the region being probed. Typically, estimates for density $n_{\mathrm{H}}$ can be found via rotational excitation analysis of observed $\mathrm{C}_{2}$ lines (Sonnentrucker et al. 2007), analysis of $\mathrm{H}$ and the $J=4$ level of $\mathrm{H}_{2}$ (Jura 1975), or from thermal pressure analysis of C I (Jenkins et al. 1983); see also Indriolo \& McCall 2012). Moreover, estimates for the fractional abundance of $\mathrm{C}$ and $\mathrm{OH}$ are required. For this proofof-concept case, we adopt fiducial values of $f_{\mathrm{H} 2}=0.053$ (Indriolo et al. 2015), ${ }^{8} x_{\mathrm{C}}=1.6 \times 10^{-4}$ (Sofia et al. 2004), and $x_{\mathrm{OH}}=1.0 \times 10^{-9}$ (Hollenbach et al. 2012). ${ }^{9}$ We discuss our model for the fractional ionization, $x_{i}$, in Section 4.1.1.

\subsubsection{Heating}

CR heating has been argued to be important in the ISM of galaxies (Field et al. 1969; Wiener et al. 2013b; Walker 2016; Owen et al. 2018, 2019b) in their circumgalactic environments (e.g., Salem et al. 2016; Owen et al. 2019a, 2019b), and even in the intra-cluster medium between galaxies (e.g., Loewenstein et al. 1991; Wiener et al. 2013a; Ruszkowski et al. 2017). Its power is mediated by the thermalization mechanism(s) at work, as governed by the local conditions (e.g., density, ionization fraction, magnetic field). Here, we outline the dominant heating processes likely to arise from the presence of CRs within MC environments.

Traditionally, the excitation and subsequent nonlinear Landau damping, ion-neutral collisional damping, and/or turbulent damping of Alfvén waves in magnetized environments, e.g., the intra-cluster medium (Loewenstein et al. 1991; Farmer \& Goldreich 2004; Fujita \& Ohira 2011; Fujita et al. 2013; Wiener et al. 2013a; Jacob \& Pfrommer 2017; Ruszkowski et al. 2017),

\footnotetext{
This is a mean value from a Milky Way sample (see Indriolo et al. 2015 for details).

9 Typically, variation of the $x_{\mathrm{OH}}$ fraction would be expected throughout an $\mathrm{MC}$ due to its formation by the recombination of $\mathrm{H}_{3} \mathrm{O}^{+}$with electrons or destruction by reactions with $\mathrm{C}$ or $\mathrm{C}^{+}$. Studies indicate that this generally would lead to a buildup of $\mathrm{OH}$ in some parts of the cloud compared to the adopted fiducial limit (Hollenbach et al. 2012). As such, the estimated $N\left(\mathrm{C}^{+}\right) / N(\mathrm{C})$ ratio in Equation (3) should be regarded as an upper limit to constrain $\zeta^{\mathrm{H}}$. Indeed, care must be taken if using this ratio alone as strong contamination by photoionization in non-shielded regions would be expected to boost the $\mathrm{C}^{+}$abundance far above the levels driven by $\mathrm{CR}$ ionization processes.
}

has been regarded as the main process by which CR thermalize, and it is likely that this would be dominant in an MC. CR heating by this channel would arise at a rate of $n_{0}\langle\sigma v\rangle \mathrm{s}^{-1}$ (Kulsrud \& Pearce 1969; Zweibel \& Shull 1982) for $\langle\sigma v\rangle$ as the rate per particle of momentum exchange between ions and neutral particles, averaged over a thermal distribution. This indicates a damping timescale of around $3 \times 10^{3} \mathrm{yr}$, which would suggest that Alfvén waves could not propagate far (less than $0.01 \mathrm{pc}$ ) into an MC (see Martin et al. 1997), even if considering artificially favorable conditions for wave propagation, i.e., low-density clouds $\left(n \approx 10^{2} \mathrm{~cm}^{-3}\right)$ appropriate for peripheral regions yet a relatively strong $0.1 \mathrm{mG}$ magnetic field more suitable for a dense core (e.g., Crutcher 2012). At higher temperatures, this damping length would be even shorter, so thermalization via this mechanism deep within an MC would depend on Alfvén amplification by those CRs able to reach the heavily shielded cores. Amplification could be either driven by streaming instabilities, or CR anisotropies within the cloud (Kulsrud \& Pearce 1969; Wentzel 1969; Zweibel $\&$ Shull 1982), or any mechanical processes (e.g., motions due to gravitational collapse/condensation within the cloud) or turbulence that may act as a wave source (Carlberg \& Pudritz 1990; McKee \& Zweibel 1995; Gammie \& Ostriker 1996; Martin et al. 1997; Falceta-Gonçalves et al. 2003).

The timescale of magnetohydrodynamical (MHD) wave excitation via the streaming instability is $\sim 10^{6} \mathrm{~s}$ (Ginzburg \& Syrovatskii 1964; Kulsrud \& Cesarsky 1971), which is shorter than the corresponding damping rate. Alfvén waves are therefore expected to persist, build up, and thermalize within an $\mathrm{MC}$, with a power of

$$
\mathcal{Q}_{\mathrm{A}}=\left|v_{\mathrm{A}, i} \cdot \nabla P_{\mathrm{c}}\right|
$$

(Wentzel 1971; Wiener et al. 2013b), where $\nabla P_{\mathrm{c}}$ is the (local) $\mathrm{CR}$ pressure gradient and $\boldsymbol{v}_{\mathrm{A}, i}$ is the generalized modified Alfvén velocity, which is defined by

$$
v_{\mathrm{A}, i}=v_{\mathrm{A}}\left(1+\varepsilon_{i}\right)^{-1 / 2}
$$

(e.g., Martínez-Gómez et al. 2018), where $\varepsilon_{i}=n_{\mathrm{H}} / n_{\text {ions }}$ and $v_{\mathrm{A}}$ $\left(=\left|v_{\mathrm{A}}\right|=B / \sqrt{4 \pi \rho}\right.$, with $\rho=\sum_{i} n_{i} m_{i}$ as the mass density, for number density $n_{i}$ of particles with mass $m_{i}$ ). We may write $n_{\mathrm{H}}=n_{\text {ions }}\left(1-x_{i}\right) / x_{i}$ as the number density of neutral molecules (regardless of whether they are $\mathrm{H}$ or $\mathrm{H}_{2}$ ), where $x_{i}$ is the ionization fraction, and $n_{\text {ions }}$ is the number density of ions (assuming their abundance is equivalent to that of thermal electrons). From this, it follows that $v_{\mathrm{A}, i} \approx v_{\mathrm{A}} \sqrt{x_{i}}$.

Given that CRs propagate preferentially along the magnetic field vectors, $\left|\nabla P_{\mathrm{c}}\right|$ in this direction can be estimated from the number density and spectrum derived from the relevant transport equation (accounting for both proton and electron contributions), with $v_{\mathrm{A}, i}$ also calculated according to the local conditions. The heating power at a location $s$ is then

$$
\mathcal{Q}_{\mathrm{A}}(s)=\left.\left(\gamma_{\mathrm{A}}-1\right)\left\{v_{\mathrm{A}, i} \int d E\left|\nabla_{\|} n(E)\right| E\right\}\right|_{s} .
$$

Here, we set the adiabatic index $\gamma_{A}=4 / 3$ for the CRs. The integral over $n(E) E d E$ gives the CR energy density, and we retain $n$ in its general form to denote the total contribution from both CR protons $\left(n_{\mathrm{p}}\right)$ and electrons $\left(n_{\mathrm{e}}\right)$.

A CR population can also heat a medium by collisional ionization and subsequent thermalization. In Spitzer \& Tomasko (1968) (see also, e.g., Goldsmith 2001), this was found to arise at a rate of roughly $6.3 \times 10^{-27} n_{\mathrm{H}} \mathrm{erg} \mathrm{cm}^{-3} \mathrm{~s}^{-1}$, 
where the heating power may be fully calculated using

$$
\mathcal{Q}_{\mathrm{I}}(s)=\sum_{i} f_{i} n_{i}(s) \int d E \mathcal{E}_{\mathrm{h}}(E) \zeta^{\mathrm{H}^{\prime}}(E)
$$

for $f_{i}$ as the abundance fraction of species $i$ with number density $n_{i}$ (we use this to account for the molecular versus atomic hydrogen fraction, which is of importance in this calculation), and where $\zeta^{\mathrm{H}^{\prime}}(E)$ is the total differential (per energy interval) rate of all CR ionization channels. The energy a "knock on" electron contributes toward heating its ambient gas is $\mathcal{E}_{\mathrm{h}}$, which is a function of the energy of the CR initiating the ionization event. Below a threshold of the excitation energy of an atom, being $3 / 4$ of its binding energy $E_{\mathrm{B}}(13.6 \mathrm{eV}$ for hydrogen), the full CR knock-on electron energy is available for heating: collisional excitations cannot act to absorb its energy (to reradiate as photons), while further secondary ionizations are also not possible. At higher energies between $3 E_{\mathrm{B}} / 4<E<E_{\mathrm{B}}$, a fraction of an electron energy $3 E_{\mathrm{B}} / 4$ in any collision may now be lost to an excitation event, with only the remainder $\left(E_{\mathrm{h}}=E-3 E_{\mathrm{B}} / 4\right)$ available for heating. At energies between $E_{\mathrm{B}}<E<3 E_{\mathrm{B}} / 2$, a single ionization or excitation process can proceed, with the probability of each determined by the relative weighting of the respective cross sections,

$$
\mathcal{E}_{\mathrm{h}}(E)=\frac{\sigma^{\mathrm{ex}}\left[E-g E_{\mathrm{B}}\right]+\sigma^{\mathrm{ion}}\left[E-E_{\mathrm{B}}\right]}{\sigma^{\mathrm{ex}}+\sigma^{\mathrm{ion}}},
$$

where $g=3 / 4$ (Spitzer \& Tomasko 1968), $\sigma^{\mathrm{ex}}$ is the collisional excitation cross section, proportional to the energy-specific collision strength (Osterbrock 1989), and $\sigma^{\text {ion }}$ is the ionization cross section for the process in question (P09). Given the energies of interest in this work, collisions would overwhelmingly lead to an ionization, meaning Equation (8) reduces to $\mathcal{E}_{\mathrm{h}}(E)=E-E_{\mathrm{B}}$. In the range of $3 E_{\mathrm{B}} / 2<E<7 E_{\mathrm{B}} / 4$ only one ionization may arise. However, if instead an excitation occurs then so must another and Equation (8) still applies, but with $g=3 / 2$. When $E \geqslant 7 E_{\mathrm{B}} / 4$, the situation is governed by the exact energy spectrum of the CRs, and $\mathcal{E}_{\mathrm{h}}(E)$ is determined numerically in Dalgarno \& McCray (1972), although in this work, we adopt the numerical approximation in Draine (2011) for computational efficiency. Overall, an ionization efficiency reduction would also arise to account for energy losses to other channels, which is calculated by weighting according to the respective timescales of each process.

Other mechanisms have also been proposed, but are unlikely to be as important as MHD wave damping, in particular, see Colafrancesco \& Marchegiani (2008), Ruszkowski et al. (2017), and Owen et al. (2018, 2019b), where on $0.1 \mathrm{kpc}$ scales, thermalization by Coulomb interactions in an ionized ISM is considered. In this process, secondary CRs are injected by pp interactions of CR primary protons (primary electrons would cool too quickly to propagate far from their source) to provide a channel by which CR protons can thermalize. This process can be the most effective in ionized media (see Owen et al. 2019b), but, in a predominantly neutral cloud, Coulomb thermalization would only be able to attain levels of around $10^{-31} \mathrm{erg} \mathrm{cm}^{-3} \mathrm{~s}^{-1}$ for $n_{\mathrm{H}}=10^{4} \mathrm{~cm}^{-3}$ and $x_{i}=10^{-8}$ (scaled from the result in Owen et al. 2019b).

\subsubsection{Equilibrium Temperature and Gas Cooling}

Cooling processes would also operate within MC environments, allowing an equilibrium temperature to be reached at which cooling and heating rates are comparable. Under typical dense MC conditions, gas cooling is dominated by $\mathrm{CO}$ and dust, with the latter only becoming important above densities of around $3 \times 10^{4} \mathrm{~cm}^{-3}$-below this, only $\mathrm{CO}$ cooling would operate effectively (e.g., Goldsmith 2001; Galli et al. 2002).

To calculate the $\mathrm{CO}$ cooling rates, we adopt the analytic approximation presented in Whitworth \& Jaffa (2018), which was based on data in Goldsmith \& Langer (1978). To approximate dust cooling rates, we assume a uniform dust temperature of $10 \mathrm{~K}$ through the cloud as a reasonable upper bound for these dense regions, otherwise we follow the treatment in Goldsmith (2001). This allows equilibrium temperature profiles of MCs to be determined when subjected to different intensities of CR heating.

\subsection{Cooling and Absorption of CRs}

\subsubsection{Electrons}

In each electron interaction event, only a small fraction of the particle energy is transferred. Thus, the cooling due to electron interactions is practically a continuous process. The following processes apply both to primary and secondary electrons/ positrons (without losing generality, hereafter, both $e^{+}$and $e^{-}$ are referred to as "electrons"). In a low-density plasma, the rate of cooling via electron-Coulomb interactions for ionization fraction $x_{i}$ is

$$
b_{\mathrm{C}} \approx m_{\mathrm{e}} c^{2} n_{\mathrm{H}} x_{i} c \sigma_{\mathrm{T}} \ln \Lambda
$$

(see Dermer \& Menon 2009), where $m_{\mathrm{e}}$ is the rest mass of an electron, $c$ is the speed of light, and $\sigma_{\mathrm{T}}$ is the Thomson cross section. The Coulomb logarithm is taken as $\ln \Lambda \simeq 30$. The rate of cooling due to electron bremsstrahlung (free-free) is

$$
b_{\mathrm{ff}}\left(\gamma_{\mathrm{e}}\right) \approx \alpha_{\mathrm{f}} c \sigma_{\mathrm{T}} n_{\mathrm{H}} x_{i} \gamma_{\mathrm{e}} m_{\mathrm{e}} c^{2}
$$

(see Dermer \& Menon 2009), where $\alpha_{\mathrm{f}}$ is the fine-structure constant and $\gamma_{\mathrm{e}}$ is the Lorentz factor of the electrons. Average ionization losses due to interactions with hydrogen per CR electron are given by

$$
b_{\text {ion }}\left(\gamma_{\mathrm{e}}\right)=\left[n_{\mathrm{H}} / n_{\mathrm{e}}\right]\left(1-x_{i}\right) \zeta_{\mathrm{e}}^{\text {ion }}\left(\gamma_{\mathrm{e}}\right) \gamma_{\mathrm{e}} m_{\mathrm{e}} c^{2}
$$

(Spitzer \& Tomasko 1968). Here, the differential (per energy interval) direct ionization rate by electrons ${ }^{10}$ is defined as

$$
\zeta_{\mathrm{e}}^{\text {ion }}\left(\gamma_{\mathrm{e}}\right)=n_{\mathrm{e}}\left(\gamma_{\mathrm{e}}\right) c \sum_{\mathrm{x}} \sigma_{\mathrm{e}, \mathrm{x}}^{\text {ion }}\left(\gamma_{\mathrm{e}}\right)
$$

where $\sigma_{\mathrm{e}, \mathrm{x}}^{\mathrm{i}}$ is the cross section associated with each relevant ionization process $\mathrm{x}$ between electrons and the neutral medium. Strictly, the composition of MCs is dominated by molecular hydrogen, not atomic hydrogen, and this changes the available channels through which ionization may proceed. Together with

\footnotetext{
${ }^{10}$ This does not account for so-called knock-on ionizations caused by energetic electrons ejected by a primary ionization process - these are only relevant for the efficiency of thermalization/total ionization rate experienced by the cloud.
} 
direct ionization $\left(e_{\mathrm{CR}}+\mathrm{H}_{2} \rightarrow e_{\mathrm{CR}}+\mathrm{H}_{2}^{+}+e\right)$, dissociative ionization $\left(e_{\mathrm{CR}}+\mathrm{H}_{2} \rightarrow e_{\mathrm{CR}}+\mathrm{H}+\mathrm{H}^{+}+e\right)$, and double ionization $\left(e_{\mathrm{CR}}+\mathrm{H}_{2} \rightarrow e_{\mathrm{CR}}+2 \mathrm{H}^{+}+2 e\right)$ may also occur. We follow the approach used by P09 to model the ionization cross sections in this work. ${ }^{11}$ Although this does not include the relativistic corrections introduced in later works (in particular, see Krause et al. 2015), we find the impact of including these in our calculations is negligible. The cooling rate due to MHD wave excitation arises at a rate of

$$
b_{\mathrm{MHD}}\left(\gamma_{\mathrm{e}}\right)=\left(\gamma_{\mathrm{A}}-1\right) v_{\mathrm{A}, i}\left|\nabla_{\|} n_{\mathrm{e}}\left(\gamma_{\mathrm{e}}\right)\right| \gamma_{\mathrm{e}} m_{\mathrm{e}} c^{2}
$$

(see also Equation (6), where symbols retain the same definitions). The rates for Compton and synchrotron cooling are

$$
b_{\mathrm{rad}}\left(\gamma_{\mathrm{e}}\right)=\frac{4}{3} \sigma_{\mathrm{T}} c \gamma_{\mathrm{e}}^{2} \epsilon_{i}
$$

(see, e.g., Blumenthal 1970), where $\epsilon_{i}$ is the energy density of the radiation field $\epsilon_{\mathrm{ph}}$ (Compton cooling) or magnetic field $\epsilon_{\mathrm{B}}$ ( $=B^{2} / 8 \pi$, synchrotron cooling), respectively. In MC environments, $\epsilon_{\mathrm{ph}} \ll \epsilon_{\mathrm{B}}$, so Compton cooling is insignificant. ${ }^{12}$ The total cooling rate (at some position $s$ ) is the sum of all contributing processes, where we note that ionization and MHD wave excitation losses are most important (in line with the dominance of these processes in driving CR heating in the cloud (see Section 2.3.2 for details).

\subsubsection{Protons}

Protons predominantly lose energy in $\mathrm{MC}$ environments by MHD wave excitation. The cooling rate follows that for electrons, given in Equation (13), where the CR proton energy $\gamma_{\mathrm{p}} m_{\mathrm{p}} c^{2}$ and density gradient $\nabla_{\|} n_{\mathrm{p}}\left(\gamma_{\mathrm{p}}\right)$ along the magnetic field vector are used. Additionally, protons interact with their environment either by ionization, or at kinetic energies above a threshold of $E_{\mathrm{p}}^{\text {th }}=0.28 \mathrm{GeV}$ (Kafexhiu et al. 2014), by pionproducing hadronic interactions. This hadronic threshold is the minimum energy required for the production of a pair of neutral pions, being the lowest energy particle forming in the resulting cascade, where $E_{\mathrm{p}}^{\text {th }}=0.28 \mathrm{GeV}=2 m_{\pi^{0}}+m_{\pi^{0^{2}}} / 2 m_{\mathrm{p}}$, for $m_{\pi^{0}}$ as the neutral pion rest mass and $m_{\mathrm{p}}$ as the proton rest mass.

The average proton cooling rate (per CR proton) due to collision-induced ionizations follows that for electrons, and is modeled as a cooling process arising at a rate given by

$$
b_{\text {ion }}\left(\gamma_{\mathrm{p}}\right)=\left[n_{\mathrm{H}} / n_{\mathrm{p}}\right]\left(1-x_{i}\right) \zeta_{\mathrm{p}}^{\text {ion }}\left(\gamma_{\mathrm{p}}\right) \gamma_{\mathrm{p}} m_{\mathrm{p}} c^{2}
$$

(Spitzer \& Tomasko 1968), where $n_{\mathrm{p}}$ is the number density of protons and $\gamma_{\mathrm{p}}$ is their Lorentz factor. The total differential ionization rate experienced by the cloud is defined as

$$
\zeta_{\mathrm{p}}^{\text {ion }}\left(\gamma_{\mathrm{p}}\right)=n_{\mathrm{p}}\left(\gamma_{\mathrm{p}}\right) c \sum_{\mathrm{x}} \sigma_{\mathrm{p}, \mathrm{x}}^{\text {ion }}\left(\gamma_{\mathrm{p}}\right)
$$

\footnotetext{
${ }^{11}$ P09 adopted the semiempirical expression by Rudd (1991) for the direct ionization process, the polynomial fit to data from Straub et al. (1996) (see also Liu \& Shemansky 2004) for the dissociative ionization process, and the fit to the data from Kossmann et al. (1990) for the double ionization process.

12 Other effects, e.g., triplet pp processes can arise at higher energies, but are not important in the weak radiation fields inside MCs (Schlickeiser 2002).
}

for $\sigma_{\mathrm{p}, \mathrm{x}}^{\mathrm{in}}$ as the cross section associated with each relevant ionization process $x$ between protons and neutral $\mathrm{H}_{2}$. The possible channels are direct ionization $p_{\mathrm{CR}}+\mathrm{H}_{2} \rightarrow p_{\mathrm{CR}}+$ $\mathrm{H}_{2}^{+}+e$, electron capture ionization $p_{\mathrm{CR}}+\mathrm{H}_{2} \rightarrow \mathrm{H}+\mathrm{H}_{2}^{+}$ (effectively a charge exchange process), dissociative ionization $p_{\mathrm{CR}}+\mathrm{H}_{2} \rightarrow p_{\mathrm{CR}}+\mathrm{H}+\mathrm{H}^{+}+e$, and double ionization $p_{\mathrm{CR}}+$ $\mathrm{H}_{2} \rightarrow p_{\mathrm{CR}}+2 \mathrm{H}^{+}+2 e$, which we model by adopting the cross sections in P09. ${ }^{13}$

The pion-producing events are modeled as an absorption process because a large fraction of the CR energy is transferred in a single interaction. These proceed via the major channels

$$
\mathrm{p}+\mathrm{p} \rightarrow\left\{\begin{array}{l}
\mathrm{p} \Delta^{+} \rightarrow\left\{\begin{array}{l}
\mathrm{pp} \pi^{0} \xi_{0}\left(\pi^{0}\right) \xi_{ \pm}\left(\pi^{+} \pi^{-}\right) \\
\mathrm{pp} \pi^{+} \pi^{-} \xi_{0}\left(\pi^{0}\right) \xi_{ \pm}\left(\pi^{+} \pi^{-}\right) \\
\mathrm{pn} \pi^{+} \xi_{0}\left(\pi^{0}\right) \xi_{ \pm}\left(\pi^{+} \pi^{-}\right)
\end{array}\right. \\
\mathrm{n} \Delta^{++} \rightarrow\left\{\begin{array}{l}
\mathrm{np} \pi^{+} \xi_{0}\left(\pi^{0}\right) \xi_{ \pm}\left(\pi^{+} \pi^{-}\right) \\
\mathrm{nn} 2 \pi^{+} \xi_{0}\left(\pi^{0}\right) \xi_{ \pm}\left(\pi^{+} \pi^{-}\right)
\end{array}\right.
\end{array}\right.
$$

where $\Delta^{+}$and $\Delta^{++}$baryons are the resonances (Almeida et al. 1968; Skorodko et al. 2008), and $\xi_{0}$ and $\xi_{ \pm}$are the multiplicities of the neutral and charged pions, respectively, which are increasingly formed at higher energies. The hadronic products continue their interaction processes until their energies fall below the interaction threshold $E_{\mathrm{p}}^{\text {th }}$, occurring within just a few interaction events (see Owen et al. 2018). The neutral pions decay rapidly into two $\gamma$-rays, with a branching ratio of $98.8 \%$ (Patrignani et al. 2016), on timescales of $8.5 \times 10^{-17} \mathrm{~s}$. The charged pions undergo a weak interaction, either via $\pi^{+} \rightarrow \mu^{+} \nu_{\mu} \rightarrow \mathrm{e}^{+} \nu_{\mathrm{e}} \bar{\nu}_{\mu} \nu_{\mu}$ or $\pi^{-} \rightarrow \mu^{-} \bar{\nu}_{\mu} \rightarrow \mathrm{e}^{-} \bar{\nu}_{\mathrm{e}} \nu_{\mu} \bar{\nu}_{\mu}$, with a branching ratio of $99.9 \%$ (Patrignani et al. 2016) on a timescale of $2.6 \times 10^{-8} \mathrm{~s}$. The rate at which protons are absorbed by the pp process is

$$
S_{\mathrm{p}}\left(\gamma_{\mathrm{p}}, s\right)=n_{\mathrm{H}}(s) n_{\mathrm{p}}\left(\gamma_{\mathrm{p}}\right) c \sigma_{\mathrm{p} \pi}\left(\gamma_{\mathrm{p}}\right),
$$

where $\sigma_{\mathrm{p} \pi}$ is the total inelastic pp interaction cross section, being well parameterized by

$$
\sigma_{\mathrm{p} \pi}=\left(30.7-0.96 \ln \left(\chi_{E}\right)+0.18\left(\ln \chi_{E}\right)^{2}\right)\left(1-\chi_{E}^{-1.9}\right)^{3} \mathrm{mb}
$$

(Kafexhiu et al. 2014), where $\chi_{E}=E / E_{\mathrm{p}}^{\text {th }}=\left(\gamma_{\mathrm{p}}-1\right) /$ $\left(\gamma_{\mathrm{p}}^{\text {th }}-1\right)$. Here, $E$ is the proton kinetic energy, $E_{\mathrm{p}}^{\text {th }}$ is the threshold kinetic energy for the pp interaction, and $\gamma_{\mathrm{p}}^{\text {th }}$ is the Lorentz factor of a proton at this threshold energy.

\subsection{CR Propagation}

CRs gyrate around magnetic field lines with a gyroradius of

$$
r_{\mathrm{L}}=\frac{1.07 \times 10^{-5}}{|Z|}\left(\frac{E}{100 \mathrm{MeV}}\right)\left(\frac{B}{\mu \mathrm{G}}\right)^{-1} \mathrm{pc},
$$

\footnotetext{
${ }^{13}$ P09 used the empirical fit to the cross section for direct ionization from Rudd et al. (1985), and the fit by Rudd et al. (1983) for the electron capture (charge exchange) ionization cross section. The cross sections for dissociative ionization and double ionization are taken to be equivalent to those for the corresponding electron interactions.
} 
where $E$ is the particle energy, $|Z|$ is the magnitude of the particle charge, and $B$ is the (uniform) magnetic field strength. The gyrofrequency is related to this by $\omega_{\mathrm{L}}=\beta_{\perp} c / r_{\mathrm{L}}$, where $\beta_{\perp} c$ is the particle velocity perpendicular to the magnetic field vector. In general, CRs propagating through the tangled magnetic fields of interstellar space do not experience a uniform deflectioninstead, their propagation is better described as a series of random scatterings in the magnetic field domains. From a phenomenological perspective, this may be regarded as a diffusion process with a characteristic length scale set by $r_{L}$. In an $\mathrm{MC}$, the magnetic field exhibits an ordered structure on the length scale of the cloud itself (see Section 2.1). Moreover, the size of the gyroradius for particles in typical MC environments is many orders of magnitude smaller than this structure: the gyroradius of a $100 \mathrm{MeV} \mathrm{CR}$ in a $100 \mu \mathrm{G}$ magnetic field is around $10^{-7} \mathrm{pc}$, being even smaller at lower energies. This compares with a length scale of a few tens of parsecs for an $\mathrm{MC}$, a few parsecs for clumps (with $n_{\mathrm{H}} \sim 10^{3} \mathrm{~cm}^{-3}$ ), or a few tenths of a parsec for the dense cores (with $n_{\mathrm{H}} \sim 10^{4}-10^{5} \mathrm{~cm}^{-3}$ ), i.e., many orders of magnitude larger than $r_{\mathrm{L}}$ in all cases. As such, propagating CRs would experience an effective locally uniform magnetic field vector within a cloud, and would be strongly guided along them (following a helical path gyrating around the field vector). This leads to strongly directed, anisotropic CR diffusion in the direction of the field vector, which facilitates magnetic focusing and CR entrapment in MCs.

\subsubsection{Magnetic Mirroring and Focusing}

Since CRs are constrained to gyrate and propagate along the magnetic field lines, their flux (per unit area) and any change in their number density (per unit volume) must be proportional to the density of the magnetic field lines per unit area. We quantify this using a magnetic concentration parameter, $\chi\left(\equiv B / B_{0}\right)$, as in Desch et al. (2004). This parameter is the ratio of the magnetic field strength $B$ measured at some location within the MC (including the core region) compared to the mean ISM value, $B_{0}$. This follows from the magnetic field strength being defined as the magnetic flux through a point, which is proportional to the concentration of magnetic field lines through that point. As a $\mathrm{CR}$ propagates along a curved magnetic field vector into an $\mathrm{MC}$, kinetic energy and magnetic moment must both be conserved. If it enters the cloud with a pitch angle (the angle between the incoming particle's velocity and the orientation of the magnetic field vector) $\theta_{\text {in }}$ and speed $v_{\text {in }}$, the component of its velocity along the field vector would be $v_{\|}=v_{\text {in }} \tilde{\mu}_{\text {in }}$, where $\tilde{\mu}_{\text {in }}$ retains its earlier definition $\left(\cos \theta_{\text {in }}\right)$. The CR gyration velocity around the magnetic field would then follow as $v_{\perp}=v_{\text {in }}\left(1-\tilde{\mu}_{\text {in }}^{2}\right)^{1 / 2}$. When a CR has propagated into a cloud where magnetic field strength has attained $B=\chi B_{0}$, the cosine of its corresponding pitch angle must be

$$
\tilde{\mu}^{2}=1-\chi+\chi \tilde{\mu}_{\text {in }}^{2},
$$

in order to ensure that kinetic energy $\propto v_{\perp}^{2}+v_{\|}^{2}$ and magnetic moment $\propto v_{\perp}^{2} / B$ are conserved. It therefore follows that $\tilde{\mu}^{2}<0$ (i.e., the $\mathrm{CR}$ will be deflected and unable to propagate into the core) unless $\tilde{\mu}_{\text {in }}^{2}>1-1 / \chi$. This effect reduces the overall flux of CRs penetrating into the core of an MC, with CRs generally being reflected out of regions where $B \geqslant \chi B_{0}$ unless the pitch angle at which they enter the cloud is small (so-called magnetic mirroring). Detailed treatments accounting the effect of mirroring and focusing in evolving magnetic fields are considered in the literature, in particular, see Kulsrud \& Pearce (1969) and Felice \& Kulsrud (2001). In our approach we consider a simple, idealized system with non-evolving magnetic fields as a demonstrative study. More sophisticated prescriptions of $\mathrm{CR}$ propagation fall beyond the scope of this first model, and we leave these to future work. We instead adopt the treatment outlined in Desch et al. (2004) to characterize the approximate combined impact of magnetic mirroring and focusing by averaging over pitch angles of CRs within and outside the cloud, and apply this as an adjustment factor to the CR distributions we later calculate (see Section 2.4.2). In this approach, the ratio of the internal and external fluxes demonstrates the degree to which magnetic mirroring/focusing has modified the CR flux at a given point where the magnetic field strength is known. Within the ISM, the angle-averaged CR flux (for $n$ as CR particle density) may be expressed as

$$
\left.\left\langle\frac{\partial n}{\partial t}\right\rangle\right|_{\mathrm{ISM}}=\int_{\Omega} \frac{d \Omega}{4 \pi} \frac{\partial n}{\partial t}=\frac{\partial n}{\partial t} \int_{-\pi}^{\pi} \frac{d \phi}{4 \pi} \int_{0}^{1} d \tilde{\mu}=\frac{1}{2} \frac{\partial n}{\partial t},
$$

assuming that the external ISM CR flux is isotropic, and only the fraction of CRs directed within a solid angle of $\Omega_{\text {in }}$ toward the cloud are able to enter it. The same analysis through some boundary within the MC (where the propagation is now anisotropic) yields

$$
\begin{aligned}
\left.\left\langle\frac{\partial n}{\partial t}\right\rangle\right|_{\mathrm{MC}} & =\int_{\Omega} d \Omega \xi\left(\tilde{\mu}_{\mathrm{in}}, \tilde{\mu}\right) \frac{\partial n}{\partial t} \\
& =\frac{\partial n}{\partial t} \int_{-\pi}^{\pi} d \phi \int_{0}^{1} d \tilde{\mu} \xi\left(\tilde{\mu}_{\mathrm{in}}, \tilde{\mu}\right),
\end{aligned}
$$

where the term $\xi\left(\tilde{\mu}_{\text {in }}, \tilde{\mu}\right)$ accounts for the combined focusing and mirroring effects, with the effective CR flux being amplified by focusing through a factor of $B_{0} \chi \tilde{\mu}$ and reduced by mirroring through a factor of $B_{0} \chi \tilde{\mu}_{\text {in }}$. By substituting Equation (21), $\xi$ may be expressed as

$$
\xi\left(\tilde{\mu}_{\mathrm{in}}, \tilde{\mu}\right)=\frac{\chi \tilde{\mu}}{\sqrt{\chi \tilde{\mu}^{2}-\chi+\chi^{2}}}
$$

such that Equation (23) can be written as

$$
\begin{aligned}
\left.\left\langle\frac{\partial n}{\partial t}\right\rangle\right|_{\mathrm{MC}} & =2 \pi \frac{\partial n}{\partial t} \int_{0}^{1} \frac{\chi \tilde{\mu} d \tilde{\mu}}{\sqrt{\chi \tilde{\mu}^{2}-\chi+\chi^{2}}} \\
& =2 \pi\left[\chi-\sqrt{\chi^{2}-\chi}\right] \frac{\partial n}{\partial t} .
\end{aligned}
$$

The magnetic scaling factor applicable to the CR flux and/or number density is then the ratio of the angle-averaged result inside the cloud (Equation (25)) compared to that outside the cloud (Equation (22)), defined as

$$
\eta(\chi)=4 \pi\left[\chi-\sqrt{\chi^{2}-\chi}\right],
$$

which is adopted in our subsequent calculations. 


\subsubsection{The Transport Equation}

If temporarily ignoring magnetic field mirroring/focusing effects, the propagation of CRs can be described using the transport equation,

$$
\begin{aligned}
& \frac{\partial n}{\partial t}-\nabla \cdot[D(E, s) \nabla n]+\nabla \cdot[v n] \\
& +\frac{\partial}{\partial E}[b(E, s) n]=Q(E, s)-S(E, s),
\end{aligned}
$$

(e.g., Schlickeiser 2002), where $n=n(E, s)$ is the differential number density of CRs (number of CR particles per unit volume per energy interval between $E$ and $E+d E$ ) at a location $\boldsymbol{s}$. The diffusive term $\nabla \cdot[D(E, s) \nabla n]$ is governed by the coefficient $D(E, s)$ which depends on the gyro-scattering radius (or frequency) of the CRs of energy $E$ in their local magnetic field (see Equation (20)), as well as turbulence and MHD perturbations along the local magnetic field vectors. We deal with this empirically in Section 3. The second propagation term $\nabla \cdot[v n]$ is usually an advection term, which describes the propagation of CRs in the bulk flow of a magnetized medium (e.g., inflow/outflow). However, in this work, it describes the propagation of CRs through a magnetized ISM (Zone 1 in Figure 1), which due to the CR streaming instability (Wentzel 1974; Kulsrud 2005), typically corresponds to the Alfvén speed (e.g., Commerçon et al. 2019), $v_{\mathrm{A}} \cdot{ }^{14}$ As the cloud becomes more neutral, the generalized modified Alfvén speed is adopted, which accounts for ionization fraction, $x_{i}$ (see Equation (5)).

The mechanical and radiative cooling term $b(E, s)$ can also be considered as an advection of the CR ensemble in energy space due to cooling processes arising along their propagations. ${ }^{15}$ The injection of CRs by the source term is given by $Q$ $(E, s)$, while CR absorption/attenuation is encoded in the sink term $S(E, s)$. As with the cooling term, the exact form of these source/sink terms depends on the CR species in question. The transport equation takes a different form for both CR protons (denoted as $n_{\mathrm{p}}$ ) and electrons (denoted $n_{\mathrm{e}}$, although, where necessary, we differentiate between primary and secondary electrons as $n_{\mathrm{e}, 1}$ and $n_{\mathrm{e}, 2}$, respectively, with the total electron number density $\left.n_{\mathrm{e}}=n_{\mathrm{e}, 1}+n_{\mathrm{e}, 2}\right){ }^{16}$

Following Owen et al. (2019b), we consider the transport equation for protons and electrons separately, where the absorption of the protons is dominated by pp losses (see Owen et al. 2018). This process injects some of the secondary CRs

\footnotetext{
14 Strictly, this is only true for particles of tens of MeVs, with high-energy particles of energies above $10 \mathrm{GeV}$ undergoing free-streaming without experiencing any substantial scattering at all (Chernyshov et al. 2018). However, we argue that at $\mathrm{GeV}$ energies and below, where the bulk of the energy density lies in our adopted CR spectrum (and where the effects of the CRs are correspondingly strongest) this treatment is sufficiently informative for our first model. We leave a more detailed model accounting for the transition from streaming to advection to future work.

15 Cooling effects are most severe for CR electrons. The CR protons, being of larger mass, have a substantially smaller Thomson cross section compared to the electrons. As such, proton cooling in this work-apart from ionization losses-is neglected.

${ }^{16}$ It is argued that both primary and secondary CRs contribute to the CR electron component of Galactic CRs and also in ISM environments of nearby galaxies. Of these, as much as $60 \%-80 \%$ could be secondary CRs (e.g., Torres 2004; Thompson et al. 2007; Lacki et al. 2010; Lacki \& Beck 2013). In this work, we regard primary CRs as those which enter the MC through the boundary, so both contributions are taken into account in our definition of the primary flux. In this paper, secondary CRs are considered as those that are produced within the MC.
}

into the source term of the transport equation for electrons (others being provided by ionizations). We adopt a Cartesian geometry, with a coordinate $s$ for the distance into an MC from a boundary set as the edge of the region of influence of the cloud (similar to, e.g., Morlino \& Gabici 2015 and Phan et al. 2018). To account for the deflective effects of magnetic mirroring and focusing on the CR distribution (see Section 2.4.1), we multiply the solution of Equation (27) by the adjustment factor $\eta(\chi)$ (Equation (26)).

\subsubsection{Primary Protons}

For protons in typical cloud environments, the timescale of radiative loss (of order gigayears) is generally longer than that of advection (approximately tens of thousands of years) and diffusion (approximately a few thousands of years). The cooling is therefore due to their ionizations of the cloud medium only. We consider no additional sources of CR protons within or in the vicinity of the MC environment, and ignore $\mathrm{CR}$ acceleration in the system. The absorption is dominated by the hadronic (pp) interaction, implying that the transport equation is simply

$$
\begin{aligned}
\frac{\partial n_{\mathrm{p}}}{\partial t} & -\frac{\partial}{\partial s}\left\{D\left(E_{\mathrm{p}}, s\right) \frac{\partial n_{\mathrm{p}}}{\partial s}\right\}+v_{\mathrm{A}, i} \frac{\partial n_{\mathrm{p}}}{\partial s} \\
& +\frac{\partial}{\partial E_{\mathrm{p}}}\left[b_{\mathrm{p}}\left(E_{\mathrm{p}}, s\right) n_{\mathrm{p}}\right] \\
= & Q_{\mathrm{p}}\left(E_{\mathrm{p}}, s\right)-n_{\mathrm{H}}(s) \sigma_{\mathrm{p} \pi}\left(E_{\mathrm{p}}\right) n_{\mathrm{p}} c
\end{aligned}
$$

where $\sigma_{\mathrm{p} \pi}$ is the hadronic (pp) interaction cross section responsible for pion production (see Section 2.3.2), and the cooling term is given by ionization loss (see Equation (16)). We apply the mirroring/focusing term $\eta(\chi)$ as an adjustment to the resulting solution, so a mirroring term does not appear in the transport equation. In the steady state, Equation (28) becomes

$$
-\frac{\partial}{\partial s}\left\{D \frac{\partial n_{\mathrm{p}}}{\partial s}\right\}+v_{\mathrm{A}, i} \frac{\partial n_{\mathrm{p}}}{\partial s}=-\frac{\partial}{\partial E_{\mathrm{p}}}\left[b_{\mathrm{p}} n_{\mathrm{p}}\right]-n_{\mathrm{H}} \sigma_{\mathrm{p} \pi} n_{\mathrm{p}} c
$$

implying a balance between cooling, absorption, diffusion, and advection of the energy carried by the CR particles. The source term is treated as a boundary condition at $s=0$. This may be solved numerically subject to appropriate boundary conditions, as outlined in Appendix A, after which the magnetic mirroring/ focusing factor $\eta(\chi)$ is applied to yield the final particle distribution. Note that, in solving Equation (29), the same boundary condition applies symmetrically at $s=s_{\mathrm{c}}$, where $s_{\mathrm{c}}$ is the size of the region of influence of the cloud. Strictly, this boundary should be taken at $\pm \infty$ as the presence of the cloud affects the CR intensity in the vicinity of the cloud (Cesarsky \& Volk 1978; Morfill 1982a). However, since such a boundary condition could not yield a self-consistent determination of the CR flux flowing through some boundary, Morfill (1982b) argued that practically each cloud may be considered to have its own sphere of influence from which the boundary condition may be taken. In this case, we take $s_{\mathrm{c}}=5 \mathrm{pc}$ to reflect the approximate separation of clouds and/or filaments found in the types of astrophysical setting in which we would expect our model to apply (see, e.g., Arzoumanian et al. 2011; Wang et al. 2020 from which distances between filaments in the IC 5146 
MC complex can be estimated to be just a few parsecs apart). A further condition is required on $D \partial n_{\mathrm{p}} / \partial s$, which is taken to be the CR flux through the boundary as estimated by $j(E)$ (see Section 2.5). We provide the resulting CR spectrum at different locations in the cloud in Appendix B.

\subsubsection{Primary Electrons}

We refer to primary electrons as those that enter the cloud through some model boundary-these could be injected into the ISM as secondaries beyond the limits of the model, but we do not require these to be distinguished as such. The form of the electron transport equation in this case is similar to that for the protons, with the exception that there is now no absorption term (electrons would cool much more rapidly than protons and are not subject to catastrophic processes like the pp interaction). In the steady state, this gives

$$
-\frac{\partial}{\partial s}\left\{D \frac{\partial n_{\mathrm{e}, 1}}{\partial s}\right\}+v_{\mathrm{A}, i} \frac{\partial n_{\mathrm{e}, 1}}{\partial s}=-\frac{\partial}{\partial E_{\mathrm{e}}}\left[b_{\mathrm{e}} n_{\mathrm{e}, 1}\right],
$$

where $D$ and $v_{\mathrm{A}}$ are the same as for protons (since they are relativistic (see, e.g., Kulsrud 2005), and the electron cooling term is the sum of all relevant contributions, given in Section 2.3.1. Equation (30) may be solved numerically (see Appendix A) subject to boundary conditions at $s=0$ and $s=s_{\mathrm{c}}$. Again, the resulting spectrum at different locations in the cloud is shown in Appendix B, which includes the adjustment to account for the magnetic mirroring/focusing.

\subsubsection{Secondary Electrons}

We consider secondary electrons as those that are injected within the MC environment, at a rate encoded by the source term $Q_{\mathrm{e}}\left(E_{\mathrm{e}}, s\right)$. In this case, the transport equation reduces to

$$
-\frac{\partial}{\partial s}\left\{D \frac{\partial n_{\mathrm{e}, 2}}{\partial s}\right\}+v_{\mathrm{A}, i} \frac{\partial n_{\mathrm{e}, 2}}{\partial s}=-\frac{\partial}{\partial E_{\mathrm{e}}}\left[b_{\mathrm{e}} n_{\mathrm{e}, 2}\right]+Q_{\mathrm{e}} .
$$

The cooling terms retain their definitions from Equation (30), while the injection term is mediated by the solution to the proton transport equation. We relate the injection of $\mathrm{CR}$ electron secondaries $Q_{\mathrm{e}}^{\text {had }}$ to the local number density and interaction rates of the $\mathrm{CR}$ protons, and we refer the reader to Owen et al. (2019b) for discussions on multiplicities, byproducts, and energy transfer efficiencies from primary to secondary species. We note that we convert the pp injection term into differential units of electron energy, ${ }^{17}$ instead of in terms of the energy of the initiating proton flux. The inclusive pion formation cross sections are adopted from Blattnig et al. (2000). Moreover, to simplify the computation (since we do not require detailed particle spectra), we assume the pion and muon

\footnotetext{
17 This assumes that multiple secondaries produced in a given interaction would have a roughly equal share of energy. This follows from the presence of a strong peak in the differential production cross section in the pp interaction (Murphy et al. 1987; Berrington \& Dermer 2003), indicating that many of the electrons are produced at similar energies. This is particularly the case at the dominating energy range of our calculation, which typically leads to the injection of electrons at a few tens to hundreds of MeVs, given that the secondary electrons characteristically inherit a few percent of the energy of the primary proton (Owen et al. 2018)
}

decay processes yield secondaries of equal energies, instead of calculating their energy distribution in full with the secondary electron and primary proton Lorentz factors related by $\gamma_{\mathrm{e}}=\gamma_{\mathrm{p}} m_{\mathrm{p}} \bar{\kappa}_{\pi} / 4 m_{\mathrm{e}}$ (Sikora et al. 1987), where the average pion-production inelasticity is $\bar{\kappa}_{\pi}=4 m_{\pi} / m_{\mathrm{p}}$; for $m_{\mathrm{e}}, m_{\pi}$, and $m_{\mathrm{p}}$ as the electron, (charged) pion, and proton rest masses, respectively. Electrons may also be injected by so-called knock-on production, where the ionization of the ambient MC gas leads to the emission of an electron of sufficiently high energy to cause further ionizations. We represent this with the knock-on injection term

$$
Q_{\mathrm{e}}^{\mathrm{K}}\left(E_{\mathrm{e}}\right)=1.75 n_{\mathrm{H}}(s) \int_{E_{1}} d E_{1} \sigma_{\mathrm{H}}^{\mathrm{ion}}\left(E_{\mathrm{e}} ; E_{1}\right) c n_{1}\left(E_{1}\right)
$$

(Brunstein 1965; Brown \& Marscher 1977), where $n_{1}$ is the differential number density (i.e., per energy interval) of CRs of energy $E_{1}$, which initiate the first ionization-either protons or electrons (including those provided by the $\mathrm{pp}$ process for completeness, although from comparison of cross sections, this would be substantially less important in an MC environment except at very high energies when $\mathrm{CR}$ fluxes would be relatively small), and $\sigma_{\mathrm{H}}^{\mathrm{ion}}\left(E_{\mathrm{e}} ; E_{1}\right)$ is the cross section for the production of knock-on electrons of energy $E_{\mathrm{e}}$ due to an initial CR energy $E_{1}$ (see Abraham et al. 1966, although we use the energy-integrated form here). This gives the contribution of knock-on secondary electrons per unit volume per energy interval between $E_{\mathrm{e}}$ and $E_{\mathrm{e}}+d E_{\mathrm{e}}$, with the total secondary CR electron injection term $Q_{\mathrm{e}}=Q_{\mathrm{e}}^{\mathrm{had}}+Q_{\mathrm{e}}^{\mathrm{K}}$. Equation (31) can then be solved (see Appendix A) subject to the boundary conditions that both $n_{\mathrm{e}}$ and $D \partial n_{\mathrm{e}} / \partial s=0$ at $s=0$ and $s=s_{\mathrm{c}}$ for all energies (as no secondary electrons would be expected to be flowing through or be present at the boundaries), with the adjustment factor $\eta(\chi)$ to account for magnetic mirroring/ focusing being applied to the solution of Equation (30) to account for this effect in the final particle distribution.

\subsection{CR Spectrum}

The irradiating incident CR spectrum is used as a boundary condition for Equation (29), and is split into two components based on their spectral shape (e.g., see P09). We note that the high-energy component is largely responsible for hadronic interactions, while that below a $\mathrm{GeV}$ is more important in directly driving ionization processes. The differential spectrum of CRs above a $\mathrm{GeV}$ observed in the Milky Way follows a distinctive power law,

$$
n_{i}(E)=\frac{d n_{i}(E)}{d E}=\tilde{n}_{\mathrm{HE}, i}\left(\frac{E}{E_{0}}\right)^{-\Gamma_{\mathrm{HE}, i}},
$$

for species $i$ being electrons or protons, and $n_{i}$ being their volume density. The spectral index in the $1 \mathrm{GeV}-1 \mathrm{PeV}$ regime (largely attributed to internal Galactic CR sources, and where the vast majority of the CR energy density lies) may be characterized by $\Gamma_{\mathrm{HE}, \mathrm{p}}=2.7,{ }^{18}$ being appropriate for primary protons (e.g., Kotera \& Olinto 2011), or the steeper index of

\footnotetext{
18 Note that the index would be less steep in regions of "fresh" CR acceleration, e.g., in the Galactic ridge or a starburst galaxy (e.g., Aharonian et al. 2006; Gaggero et al. 2017; H.E.S.S. Collaboration et al. 2018a, 2018b)
} 
$\Gamma_{\mathrm{HE}, \mathrm{e}}=3.3$ for primary electrons (e.g., Hillas 2006). The spectral index of secondary electrons is determined by the solution of the injection/transport equation rather than being adopted as an intrinsic boundary condition. We specify $\tilde{n}_{\mathrm{HE}, i}$ as the normalization of the high-energy component

$$
\tilde{n}_{\mathrm{HE}, i}=\frac{\epsilon_{\mathrm{CR}, i} f_{\mathrm{U}}\left(2-\Gamma_{\mathrm{HE}, i}\right) E_{0}^{-\Gamma_{\mathrm{HE}, i}}}{E_{\max }^{2-\Gamma_{\mathrm{HE}, i}}-E_{0}^{2-\Gamma_{\mathrm{HE}, i}}},
$$

with $E_{\max }=1 \mathrm{PeV}$, and the reference energy taken as $E_{0}=1 \mathrm{GeV} . \epsilon_{\mathrm{CR}, i}$ is the $\mathrm{CR}$ energy density across all components, which takes a value of around $1.8 \mathrm{eV} \mathrm{cm}^{-3}$ for the Galactic ISM, of which (roughly) around $0.4 \mathrm{eV} \mathrm{cm}^{-3}$ may be attributed to the component below $1 \mathrm{GeV}$ and the rest to higher-energy CRs (e.g., Webber 1998; Ferrière 2001). The parameter $f_{\mathrm{U}}=1.4 / 1.8$ is the fraction of CR energy density attributed to the high-energy component of the spectrum, with the remainder at lower energies being described by

$$
n_{i}(E)=\tilde{n}_{\mathrm{LE}, i}\left(\frac{E}{E_{0}}\right)^{-\Gamma_{\mathrm{LE}, i}} .
$$

The normalization $\tilde{n}_{\mathrm{LE}, i}$ is specified by the high-energy spectrum at $1 \mathrm{GeV}$ (i.e., at $E_{0}$ ) to ensure continuity across the spectral break. The spectral continuity is a more physically meaningful condition than maintaining an exact energy density ratio between the two $\mathrm{CR}$ components, which is subjected to substantial uncertainties as a result of, e.g., modulation effects in the solar neighborhood and spatial inhomogeneities (e.g., Webber 1998; Cummings et al. 2016). The ratio $n_{\mathrm{e}} / n_{\mathrm{p}}$ at $1 \mathrm{GeV}$ is set to be $1 \%$, in line with observations (e.g., see Hillas 2006). We set two values for each of $\Gamma_{\mathrm{LE}, \mathrm{e}}$ and $\Gamma_{\mathrm{LE}, \mathrm{p}}$ to account for the broad range of spectral indices considered in literature. The "minimum" spectra use $\Gamma_{\mathrm{LE}, \mathrm{p}}=-0.95$ (Webber 1998, hereafter W98) and $\Gamma_{\mathrm{LE}, \mathrm{e}}=-0.08$ (the "conventional" model C of Strong et al. 2000, hereafter, C00, following the notation of P09). The "maximum" spectra use $\Gamma_{\text {LE, }}=1$ (Moskalenko et al. 2002, hereafter M02) and $\Gamma_{\mathrm{LE}, \mathrm{e}}=1$ (the model SE in Strong et al. (2000), hereafter E00 by the P09 convention). We also adopt a CR flux model $j(E)$ based on the above treatment, normalized to that of P09 for Milky Way conditions to provide an appropriate flux boundary condition when later solving the transport Equations (29) and (30).

\section{Magnetic Field Structure and CR Propagation}

\subsection{Empirical Characterization of CR Propagation}

We adopt the same approach as Schlickeiser \& Achatz (1993a, 1993b) (see also Schlickeiser 2002; Kulsrud 2005) to characterize CR propagation in the MCs. In this approach, the background cloud-scale magnetic field structure is taken to vary on length scales that are substantially larger than both the magnetic field fluctuations and gyrating (scattering) radii of CRs. Using a quasi-linear approximation (Jokipii 1966; Schlickeiser 2002), the Fokker-Planck (FP) equation (Kirk et al. 1988) can be greatly simplified, assuming that (i) the turbulence driving the field fluctuations is purely magnetic ${ }^{19}$ and of low frequency, (ii) the turbulence components on

\footnotetext{
${ }^{19}$ Essentially, this means neglecting density perturbations as well as the electric field component of associated Alfvén waves.
}

different scales are uncorrelated and noninteracting, i.e., $v_{\mathrm{A}} \ll c$, and (iii) the CR pitch angle is small. When the flow of CRs and the orientation of the fluctuations are largely parallel to the orientation of the background cloud-scale magnetic field vector and independent of that in the perpendicular direction, the only nonvanishing FP coefficient is then

$$
P_{\mu \mu} \approx \frac{\mathcal{J}\left(\lambda_{1}\right)}{v_{\mathrm{A}} \lambda_{1}}\left(\frac{\omega_{\mathrm{L}} B_{0}}{B}\right)^{2} \mathcal{I}_{\perp} .
$$

Here, $\lambda_{1}=\lambda_{\mathrm{td}}\left(\left|\omega_{\mathrm{L}}\right| / \omega_{\mathrm{p}, 0}\right)$ is the CR resonant scattering length scale parallel to the background magnetic field line. The turbulent decay length scale is $\lambda_{\mathrm{td}} \approx v_{\mathrm{A}} \tau_{\mathrm{td}}$, where we adopt a turbulent decay timescale of $\tau_{\mathrm{td}}=2 \mathrm{Myr}$ in our calculations (see Gao et al. 2015; Larson et al. 2015). The CR gyrofrequency is $\omega_{\mathrm{L}}$ with a sign convention set by the charge (in units of proton charge). In the relativistic limit, this is given by $\omega_{\mathrm{L}}=\left(10^{1} / \gamma_{\mathrm{p}}\right)(B / 1 \mathrm{mG}) \mathrm{s}^{-1}$ for protons, and $\omega_{\mathrm{L}}=\left(1.8 \times 10^{4} / \gamma_{\mathrm{e}}\right)(B / 1 \mathrm{mG}) \mathrm{s}^{-1}$ for electrons (see, e.g., Kulsrud 2005). The normalization $\omega_{\mathrm{p}, 0}$ is taken to be the gyrofrequency of a CR proton at a reference energy of $100 \mathrm{MeV}$. We adopt a magnetic field strength normalization of $B_{0}=1 \mathrm{mG}$ to be comparable to the magnetic field strength outside the densest parts of clumps/cores (see, e.g., Crutcher et al. 2010; Li et al. 2015). Hence, what remains to be evaluated are the two variables $\mathcal{J}\left(\lambda_{1}\right)$ and $\mathcal{I}_{\perp}$ in the above equation.

The dimensionless variable $\mathcal{J}\left(\lambda_{1}\right)$ characterizes the magnetic field fluctuations along the direction of the background largescale magnetic field vector and is defined as

$$
\mathcal{J}\left(\lambda_{1}\right) \equiv \int_{0}^{\lambda_{1}} d \lambda \frac{\lambda}{\lambda_{1}} \hat{P}_{\|}\left(k_{\mathrm{c}} \lambda\right)+\int_{\lambda_{1}}^{\infty} d \lambda \frac{\lambda_{1}}{\lambda} \hat{P}_{\|}\left(k_{\mathrm{c}} \lambda\right)
$$

where $\hat{P}_{\|}$is the power spectrum of the fluctuations along the large-scale magnetic field vector. The wavenumber normalization is defined as $k_{\mathrm{c}}=\omega_{\mathrm{p}, 0} / v_{\mathrm{A}}$. In terms of the dimensionless variable $\kappa_{\|}=\lambda k_{\mathrm{c}}$,

$$
\begin{aligned}
\mathcal{J}\left(\lambda_{1}\right)= & k_{\mathrm{c}}^{-1} \int_{0}^{\lambda_{1} k_{\mathrm{c}}} d \kappa_{\|} \frac{\kappa_{\|}}{k_{\mathrm{c}} \lambda_{1}} \hat{P}_{\|}\left(\kappa_{\|}\right) \\
& +k_{\mathrm{c}}^{-1} \int_{\lambda_{1} k_{\mathrm{c}}}^{\infty} d \kappa_{\|} \frac{k_{\mathrm{c}} \lambda_{1}}{\kappa_{\|}} \hat{P}_{\|}\left(\kappa_{\|}\right) .
\end{aligned}
$$

The variable $\mathcal{I}_{\perp}$ specifies the contribution from the orthogonal components of the magnetic field fluctuations. Note that $\mathcal{I}_{\perp}$ is not dimensionless. Without losing generality, we denote the two orthogonal components of the perpendicular wavevector by $y$ and $z$, corresponding to the wavevectors $\boldsymbol{k}_{y}$ and $\boldsymbol{k}_{z}$, respectively. We have $\boldsymbol{k}_{\perp}=\boldsymbol{k}_{y}+\boldsymbol{k}_{z}$, with $k_{\perp}^{2}=k_{y}^{2}+k_{z}^{2}$. In terms of these wavevectors and the normalization $k_{c}$,

$$
\begin{aligned}
\mathcal{I}_{\perp} & \equiv \int_{-\infty}^{\infty} \frac{d k_{y}}{k_{\mathrm{c}}} \int_{-\infty}^{\infty} \frac{d k_{z}}{k_{\mathrm{c}}} \hat{P}_{\perp}\left(k_{y}, k_{z} ; k_{\mathrm{c}}\right) \\
& =\int_{\perp} \frac{d^{2} \boldsymbol{k}_{\perp}}{k_{\mathrm{c}}^{2}} \hat{P}_{\perp}\left(\boldsymbol{k}_{\perp} ; k_{\mathrm{c}}\right),
\end{aligned}
$$

where $\hat{P}_{\perp}$ is the power spectrum of the perpendicular component of the magnetic field fluctuations, which effectively takes the form of a scalar delta function, i.e., $\hat{P}_{\perp}\left(\boldsymbol{k}_{\perp} ; k_{\mathrm{c}}\right)=\delta\left(k_{\perp}^{2}-k_{\mathrm{c}}^{2}\right)$ (see the "slab" approximation, Hasselmann \& Wibberenz 1968). 
For isotropic fluctuations in the $y-z$ plane, we have

$$
\begin{aligned}
\mathcal{I}_{\perp} & =2 \pi \int_{0}^{\infty} \frac{d k_{\perp} k_{\perp}}{k_{\mathrm{c}}^{2}} \delta\left(k_{\perp}^{2}-k_{\mathrm{c}}^{2}\right) \\
& =\pi \int_{0}^{\infty} \frac{d k_{\perp} k_{\perp}}{k_{\mathrm{c}}^{3}}\left[\delta\left(k_{\perp}+k_{\mathrm{c}}\right)+\delta\left(k_{\perp}-k_{\mathrm{c}}\right)\right] .
\end{aligned}
$$

As $k_{\mathrm{c}} \neq 0, k_{\perp}+k_{\mathrm{c}}>0$, and hence,

$$
\mathcal{I}_{\perp}=\pi \int_{0}^{\infty} \frac{d k_{\perp} k_{\perp}}{k_{\mathrm{c}}^{3}} \delta\left(k_{\perp}-k_{\mathrm{c}}\right)=\frac{\pi}{k_{\mathrm{c}}^{2}} .
$$

Finally, we may relate the spatial diffusion coefficient $D$ to $P_{\mu \mu}$, with

$$
D \approx \frac{c^{2}}{8}\left(1-\frac{1}{\gamma^{2}}\right) \int_{-1}^{1} d \mu \frac{\left(1-\mu^{2}\right)^{2}}{P_{\mu \mu}} .
$$

Since the pitch angles are small, $P_{\mu \mu}$ is not strongly dependent on $\mu$ (see Schlickeiser \& Achatz 1993b), and we can take it outside the integral. Thus, we obtain $D \approx 2 c^{2} / 15 P_{\mu \mu}{ }^{20}$ for $\gamma \gg 1$. Although this approximation is adopted in our calculations, our results would not differ much if fully accounting for the presence of nonrelativistic particles.

\subsection{Diffusion Estimation}

\subsubsection{Angular Dispersion Function}

Dust polarization can be used to probe the magnetic field structure in dense MC environments over a range of scales. The field structure is often characterized by the angular dispersion function, defined as

$$
\mathcal{S}_{d}(\ell)=\frac{1}{N_{\text {pair }}} \sum_{i=1}^{N_{\text {pair }}}\left[\varphi_{i}(s+\ell)-\varphi_{i}(s)\right]^{d}
$$

(e.g., Redaelli et al. 2019), which is sometimes also referred to as the "structure function." Here, $d$ is the order number (in this case, we adopt $d=2$ to constrain the power spectrum), while the normalization $N_{\text {pair }}=N_{\mathrm{P}}\left(N_{\mathrm{P}}-1\right) / 2$ is the number of unique pairs in a data set of $N_{\mathrm{P}}$ individual points.

While similar to the correlation function, the structure function can be computed to higher accuracy with less data (Schulz-Dubois \& Rehberg 1981), making it more appropriate for smaller data sets with large uncertainties, such as those being used in this study. The angular dispersion function has often been used to study astrophysical magnetic fields, using, e.g., rotation measure (e.g., Minter \& Spangler 1996; Lazarian \& Pogosyan 2016; Xu \& Zhang 2016), as well as polarization angle (PA) measurements, including in the analysis of $\mathrm{MC}$ environments (e.g., Hildebrand et al. 2009; Houde et al. 2009; Planck Collaboration et al. 2016; Redaelli et al. 2019; Wang et al. 2019).

In this work, differences between pairs of measured dust PAs $\varphi$ over separations $\ell$ are computed to quantify the similarity in the orientation of magnetic field fluctuations on different scales, with the intention of encoding the deviation of local perturbations from the background mean field vector. ${ }^{21}$ In practice, to calculate $\mathcal{S}_{n}(\ell)$ from a set of $N_{\mathrm{P}}$ PAs, every unique

\footnotetext{
${ }^{20}$ Note that this formulation would be invalid if the MC is not in a steady state, i.e., if there are large-scale flows of the cloud medium, resulting from, e.g., ongoing gravitational collapse, inflows, or outflows.

21 The strength of the local field is estimated separately (see Section 3.3).
}

pair in that set must be identified and binned according to their angular separation distance $\ell$. This would yield $N_{\text {pair }}$ unique pairs, indexed sequentially as

$$
N_{\text {pair }}=j\left(N_{\mathrm{P}}-1\right)-\frac{j^{2}-j}{2}-\left(N_{\mathrm{P}}-i\right),
$$

for $i$ and $j$ as the indices of contributing $N_{\mathrm{P}}$ data points to that pair in the original data set (where $i \neq j$ ). Equation (43) would then be applied to all points within that bin to give an estimate for the angular dispersion function at that scale.

\subsubsection{Diffusion Parameter Estimation}

The application of Equation (42) to empirical data requires the computation of the parallel fluctuation term $\mathcal{J}\left(\lambda_{1}\right)$. This depends on the power spectrum $\hat{P}(k)$ of fluctuations along the large-scale magnetic field vector, which we argue is well characterized by the fluctuations in the measured PAs. The power spectrum $\hat{P}(k)$ and angular dispersion function $\mathcal{S}_{2}(\ell)$ are related (via the Wiener-Khinchin theorem; see Wiener 1930; Percival \& Walden 1993) by

$$
\hat{P}(k)=\frac{1}{2} \mathcal{F}\left[\mathcal{S}_{2}(\ell)\right]
$$

(see Appendix $\mathrm{C}$ for details), where $\mathcal{F}[\ldots]$ denotes a Fourier transform (FT). When applied to a discrete data set separated into $N_{\text {bins }}$ bins according to scale (e.g., measured PA difference between a pair of points, binned according to the angular separation of each pair (see Section 4.2), the discrete FT $^{22}$ of $S_{2}\left(\ell_{n}\right)$ can be taken for each scale bin $\ell_{n}$ to find $P\left(\kappa_{n}\right)$. The fluctuation statistic then follows a discretized form of Equation (38):

$$
\mathcal{J}\left(\lambda_{1}\right) \approx k_{c}^{-1} \sum_{n=1}^{i_{\mathrm{b}}} \frac{\kappa_{n}}{k_{c} \lambda_{1}} P\left(\kappa_{n}\right)+k_{c}^{-1} \sum_{n=i_{\mathrm{b}}}^{N_{\mathrm{bins}}} \frac{k_{c} \lambda_{1}}{\kappa_{n}} P\left(\kappa_{n}\right),
$$

where $i_{\mathrm{b}}$ is the bin index corresponding to the (normalized) resonant length scale $\lambda_{1} k_{c}$, and $P\left(\kappa_{n}\right)$ is the discrete FT of $S_{2}\left(\ell_{n}\right)$ for $\ell_{n}$ as the characteristic separation length between data pairs in the bin (taken simply as the bin center point in $\ell$ ). $\kappa_{n}$ is the normalized wavenumber associated with $\ell_{n}$. An empirical estimation for the diffusion parameter $D$ within the observed system is obtained by substituting Equation (46) into (36).

This allows us to calculate the diffusion coefficient from observations. For instance, we obtain a value of around $10^{29}$ $10^{30} \mathrm{~cm}^{2} \mathrm{~s}^{-1}$ (depending on wave band (see Appendix D for details) for a $1 \mathrm{GeV} \mathrm{CR}$ in a magnetic field of reference strength $B_{\text {ref }}=2.46 \mu \mathrm{G}$ (see Section 4.2.2) when we apply this to observations of the IC $5146 \mathrm{MC}$ complex (Section 4.2). This compares with estimates of around $10^{26} \mathrm{~cm}^{2} \mathrm{~s}^{-1}$ for MCs in the W28 region (Gabici 2011), ${ }^{23} 10^{29} \mathrm{~cm}^{2} \mathrm{~s}^{-1}$ in the Galactic Center ridge (Gabici 2011), or values of between $10^{25}$ and $10^{27} \mathrm{~cm}^{2} \mathrm{~s}^{-1}$ in the Sgr B2 giant MC (Protheroe et al. 2008; Dogiel et al. 2015). To account for the energy dependence of

\footnotetext{
${ }^{22}$ We use the discrete FT implementation in the SciPy Python package (Virtanen et al. 2020), and NumPy (Harris et al. 2020), which uses the algorithm set out in Press et al. (2007) (see also Cooley \& Tukey 1965).

${ }^{23}$ We note that this value is likely attributed to CRs in the $10 \mathrm{TeV}$ range, and would correspond to a diffusion coefficient value of around $10^{28} \mathrm{~cm}^{2} \mathrm{~s}^{-1}$ if scaled to $\mathrm{GeV}$ energies.
} 
the diffusion coefficient, we adopt the parameterized scaling

$$
D(E, s)=D_{0}\left[\frac{r_{\mathrm{L}}\left(E,\left.\langle|B|\rangle\right|_{s}\right)}{r_{\mathrm{L}, 0}}\right]^{\delta}
$$

based on the local gyroradius $r_{\mathrm{L}}$ compared to $r_{\mathrm{L}, 0}$, i.e., that of a $1 \mathrm{GeV} \mathrm{CR}$ in a reference magnetic field of characteristic mean strength $\left.B_{\text {ref. Here, }}\langle|B|\rangle\right|_{s}=|B(s)|$ is the characteristic mean magnetic field strength at some position $s$, and $D_{0}$ is the value of the characteristic diffusion coefficient calculated as outlined above. The index $\delta=1 / 2$ (see also Berezinskii et al. 1990; Strong et al. 2007) accounts for the cloud turbulence spectrum, which is set here to be the same as the broader ISM. The exact choice of this parameter does not strongly influence our results, and we leave a more careful assessment of its value in specific regions to future work.

\subsection{Observing Magnetic Fields in MCs}

Polarization of starlight by dust (as well as polarized submillimeter emission from the dust itself) is often used to probe the orientation and structure of interstellar magnetic fields on the plane of the sky (see Crutcher 2012 for a review). This polarization arises from selective absorption (or re-emission) by dust grains, whose magnetic moments tend to align themselves with the local interstellar magnetic field due to radiative torque alignment, leading to a preferred perpendicular orientation of the grains to ambient magnetic fields in low-extinction regions (Dolginov \& Mitrofanov 1976; Draine \& Weingartner 1996, 1997; Lazarian et al. 1997). As a result, a larger grain extinction cross section occurs perpendicular to the background magnetic field vector, causing linear polarization. While there is some debate over whether this alignment mechanism could operate effectively in denser high-extinction regions where radiation fields are lacking, e.g., in MCs and cores therein-results are so far largely consistent with the radiative torque model, but with substantial variation in alignment efficiency between individual sources (see Whittet et al. 2008; Cashman \& Clemens 2014). ${ }^{24}$ The strength of magnetic fields in MCs can be estimated by the Davis-Chandrasekhar-Fermi (Davis 1951; Chandrasekhar \& Fermi 1953) method, being the "standard" approach to infer magnetic field information from dust polarization data. This assumes equipartition between turbulent magnetic energy and turbulent kinetic energy such that the observed PA dispersion and velocity dispersion can be related to the magnetic field strength on the sky plane.

\section{Model and Results}

In the first instance (Section 4.1), we consider an idealized MC model to assess the propagation and interactions of CRs. The model has a simple Plummer-like density profile with a frozen-in magnetic field and a simplified CR diffusion coefficient. Our intention is to demonstrate the magnitude of the ionization and heating effects CRs may impart in such an idealized scenario. However, we acknowledge that such a treatment is not necessarily representative of all ISM MCs and star-forming regions, where $\mathrm{CR}$ diffusion can be different to that in the broader

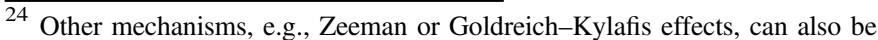
used to probe MC magnetic fields, with the Zeeman effect being the only direct method to measure the magnetic field strength (Crutcher et al. 1993). However in MCs these mechanisms are more difficult to be observed than dust polarization (see, e.g., Crutcher 2012).
}

ISM (e.g., see Dogiel et al. 2015), which finds a value of $3 \times 10^{27} \mathrm{~cm}^{2} \mathrm{~s}^{-1}$ for the diffusion coefficient in the MC Sgr B2 near the Galactic Center, around an order of magnitude lower than the typical value quoted for the ISM (see, e.g., Berezinskii et al. 1990; Aharonian et al. 2012; Gaggero 2012). As such, we later compute an empirically derived diffusion coefficient (using the method detailed in Section 3.2) specifically for the IC 5146 MC complex in Section 4.2. This uses the inferred structure of the local magnetic field estimated from dust polarization to determine a diffusion coefficient appropriate for this region. We use this to apply our model to identified filamentary structures in IC 5146 to assess a more realistic level of CR ionization and heating for a typical Milky Way star-forming environment.

\subsection{Model}

\subsubsection{Fractional Ionization and Density Profile}

The ionization of molecular gas in different cloud components may largely be considered as a balance between the CR ionization rate and recombination processes. The resulting ionization fraction per $\mathrm{H}_{2}$ molecule may be approximated by

$$
x_{i}(s)=x_{i, 0}\left(\frac{Y}{Y_{\odot}}\right)^{1 / 2}\left(\frac{\zeta^{\mathrm{H}}}{10^{-17} \mathrm{~s}^{-1}}\right)^{1 / 2}\left(\frac{n(s)}{10^{5} \mathrm{~cm}^{-3}}\right)^{-1 / 2}
$$

(Elmegreen 1979), where $x_{i, 0}=8.7 \times 10^{-8}$ for small dust grains and $Y_{\odot}$ is the solar metallicity. Strictly, the ionization fraction should be calculated dynamically in our model, but to reduce computational time we consider it sufficient to conservatively adopt a value of the "standard" $\mathrm{CR}$ ionization rate of $\zeta^{\mathrm{H}}=10^{-16} \mathrm{~s}^{-1}$, typically appropriate for the diffuse inter-clump medium (Black et al. 1978; van Dishoeck \& Black 1986; Federman et al. 1996). The density profile $n(s)$ comprises a clump and a core, described by two superimposed Plummer profiles (Whitworth \& Ward-Thompson 2001; Lee et al. 2003; Dib et al. 2010), such that $n(s)=n_{0}\left[1+\left(s / s_{0}\right)^{2}\right]^{a}$, where $-2.5 \leqslant a \leqslant-1.5$ for different clouds (Federrath \& Klessen 2013), including IC 5146 (see Arzoumanian et al. 2011). For our calculations, we adopt $a=-2$, assuming that gravitational pressure and thermal pressure are in equilibrium, with $n_{0}=10^{3} \mathrm{~cm}^{-3}$ and $s_{0}=2 \mathrm{pc}$ for the clump region, and $n_{0}=10^{5} \mathrm{~cm}^{-3}$ and $s_{0}=0.2 \mathrm{pc}$ for the core (e.g., Bergin \& Tafalla 2007).

\subsubsection{Magnetic Field and Diffusion Coefficient}

We consider the magnetic field strength to simply scale with the cloud density

$$
B(n)=\left\{\begin{array}{cc}
B_{\mathrm{ICM}}, & n \leqslant n_{1} \\
B_{\mathrm{ICM}}\left(n / n_{1}\right)^{\bar{q}}, & n>n_{1},
\end{array}\right.
$$

where we set $n_{1}=300 \mathrm{~cm}^{-3}, B_{\mathrm{ICM}}=30 \mu \mathrm{G}$ as a background reference magnetic field strength appropriate for the interclump medium, ${ }^{25}$ and $\bar{q} \approx 2 / 3$, assuming spherical collapse and magnetic flux freezing (see Mestel 1966; Crutcher et al. 2010). In this first approach (which is not specific to any

\footnotetext{
${ }^{25}$ This is estimated from line-of-sight field strengths in Crutcher et al. (2010) by omitting the diffuse cloud components (see also Thompson et al. 2019, who specifically considered inter-core regions of MCs, with $30 \mu \mathrm{G}$ as a line-of-sight magnetic field strength falling well within the suggested range, albeit stronger than average for their sample).
} 
particular MC complex), we do not account for the orientation or structure of the magnetic field, and simply assume isotropic diffusion with a simplified coefficient of the form given by Equation (47). Moreover, we adopt the empirical normalization reference for diffusion coefficient to be that based on the broader Milky Way ISM as $D_{0}=3.0 \times 10^{28} \mathrm{~cm}^{2} \quad \mathrm{~s}^{-1}$ (Berezinskii et al. 1990; Aharonian et al. 2012); Gaggero 2012) and $B_{\text {ref }}=5 \mu \mathrm{G}$.

\subsubsection{Results}

The transport Equations (29)-(31) are solved according to the scheme in Appendix A for the cloud model specified in Sections 4.1.1 and 4.1.2 to calculate the distribution of CRs in an idealized environment. Figure 2 shows the distribution of primary CRs, gradually decreasing in number density toward the cloud center, which is also reflected in the CR energy density distribution in Figure 3. The latter arises from mirroring effects, coupled with a stronger magnetic field hampering CR propagation into the central region. The decrease in $\mathrm{CR}$ abundance is relatively moderate-roughly half of ISM levels - suggesting that the combined effects of CR containment, mirroring, and focusing by $\mathrm{MC}$ environments are not particularly severe.

\subsubsection{Comment on Secondary Electrons}

The impact of the secondary CRs in this study is found to be negligible: their abundance is several orders of magnitude lower than the primary CRs and their impacts are correspondingly small. A low secondary abundance would also be consistent with the view that $\gamma$-rays from neutral pion decays would presumably dominate the high-energy emission from MCs (Brown \& Marscher 1977; Gabici et al. 2009; Casanova et al. 2010; Dogiel et al. 2018), ${ }^{26}$ and any synchrotron emission would come from the primary electron component of the CR flux (Yusef-Zadeh et al. 2013; Strong et al. 2014; Padovani \& Galli 2018). ${ }^{27}$ This is in line with expectations: knock-on production and pionproduction processes injecting secondary electrons operate fairly competitively with one another (with knock-on processes slightly dominating at lower energies). This occurs at a rate of $\dot{n}_{\mathrm{e}} \sim n_{\mathrm{H}} c \sigma_{\left[\pi^{ \pm}\right]} n_{\mathrm{p}}$ (as protons are more abundant than electrons in the primary flux), where $\sigma_{\left[\pi^{ \pm}\right]} \approx 10^{-29} \mathrm{~cm}^{2}$ at a $\mathrm{GeV}$ (Blattnig et al. 2000) is the effective pion-production cross section (note that this is different from the total pp inelastic cross section $\sigma_{\mathrm{p} \pi}$ defined in Equation (19)). This can be balanced against the "loss" of secondary electrons via cooling over a timescale of $t_{\mathrm{e}} \approx\left(\sigma^{\text {ion }} c n_{\mathrm{H}}\right)^{-1}$. Radiative processes would be more important at higher energies, but the power-law nature of the spectrum means that the number of

\footnotetext{
${ }^{26}$ Leptonic $\gamma$-rays and X-rays may also arise as a result of bremsstrahlung by the high-energy tail of the energetic electron distribution (Yusef-Zadeh et al. 2002, 2013) - but these would be attributed to primary electrons rather than locally injected secondaries.

27 Secondaries could begin to dominate at very high densities on much smaller scales, e.g., in circumstellar disks (Padovani \& Galli 2018). Very compact nonthermal sources in MCs may be a signature of synchrotron emission from secondary electrons (Jones 2014), although these may depend on in situ reacceleration in, e.g., protostellar jets (Padovani et al. 2015, 2016; Cécere et al.

2016). Note that this conflicts with earlier studies, which argued that synchrotron radiation from secondary electrons in dark clouds could actually dominate Galactic radio emission (Brown \& Marscher 1977); synchrotron emission from secondaries was also considered in Dogel \& Sharov (1990) and Jones (2014), but these studies did not compare with the emission from primary electrons.
}

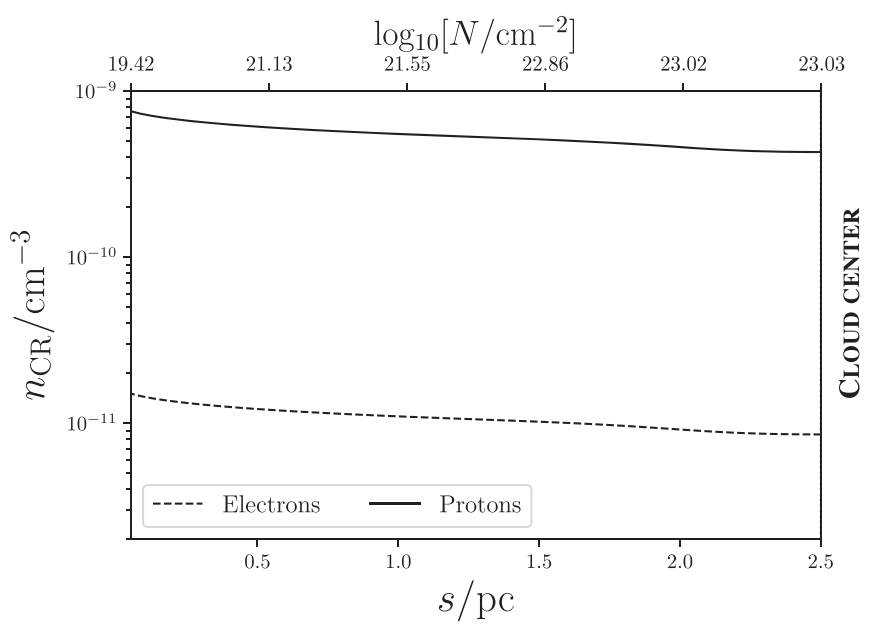

Figure 2. Distribution of energetic primary CR protons and electrons above a $\mathrm{GeV}$ propagating into the MC model prescribed in Sections 4.1.1 and 4.1.2, with distance into the cloud $s$ shown on the lower $x$-axis, and the corresponding gas column density $N$ on the upper axis. The small decrease in the CR number density toward the core is due to increased magnetic mirroring effects.

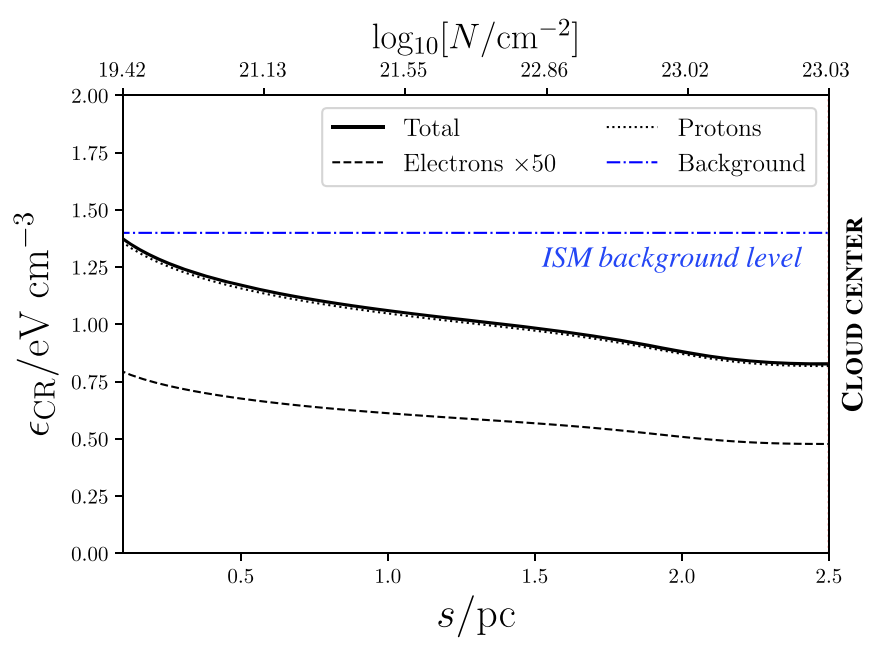

Figure 3. Energy densities of primary CRs throughout the cloud under Galactic conditions. The background interstellar CR energy density is indicated by the blue line, while the contribution from the secondary CRs is negligible (not shown). The primary electron component is multiplied by 50 in this plot for clarity.

these higher-energy electrons makes a negligible contribution to their total number density. So, lower-energy ionization losses can be regarded as the dominant secondary electron cooling process. Typically, $\sigma^{\text {ion }} \approx 10^{-20} \mathrm{~cm}^{2}$ is the electronionization cross section (P09) for a secondary electron generated by a $1 \mathrm{GeV}$ hadronic primary (retaining a few percent of the primary's energy, see Owen et al. 2018). It then follows that $n_{\mathrm{e}} \approx \dot{n}_{\mathrm{e}} t_{\mathrm{e}}$, which may be rearranged to give the ratio $n_{\mathrm{e}} / n_{\mathrm{p}} \approx \sigma_{\left[\pi^{ \pm}\right]} / \sigma^{\text {ion }} \sim 10^{-9}$, thus, confirming the negligible level of secondaries.

\subsubsection{Observable and Astrophysical Impacts}

CRs can influence their host environment by ionization, heating, and modifying the chemical balance of certain species (see, e.g., Ivlev et al. 2018). The ionization profiles due to the CR distributions in Section 4.1.3 are shown in Figure 4. The impact of the high-energy cosmic-ray (HECR) component, 


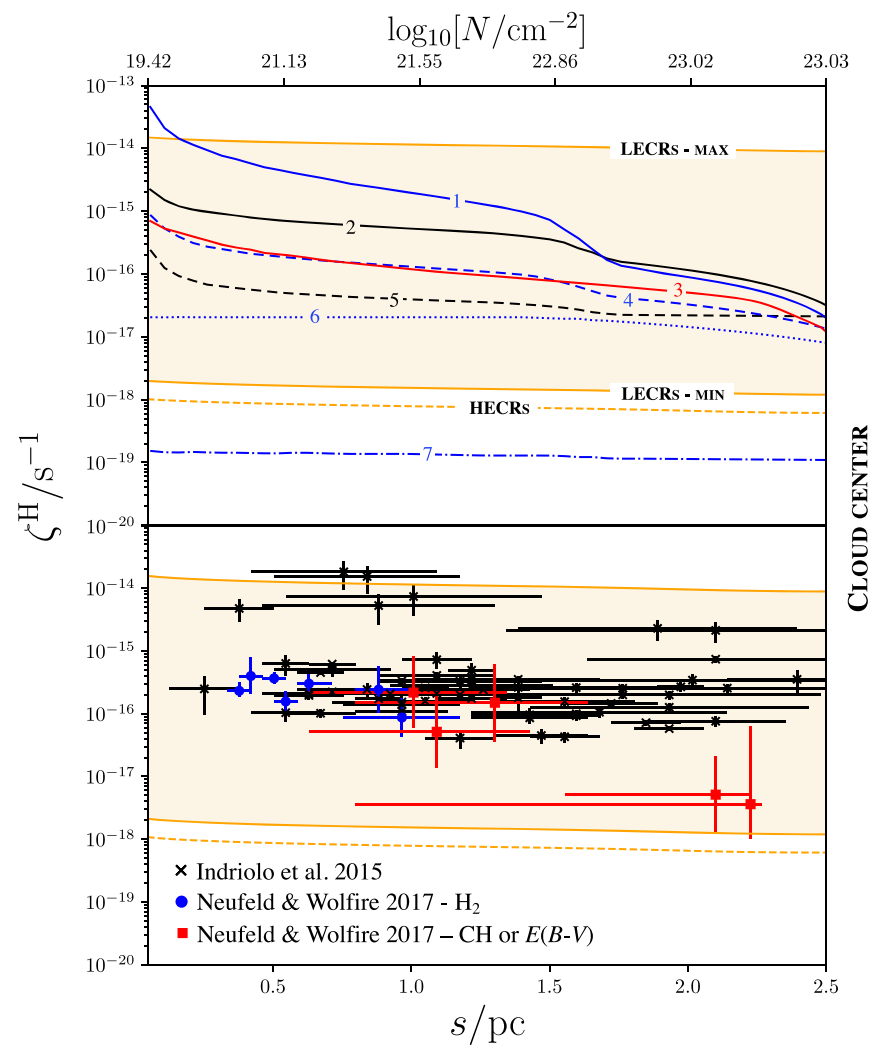

Figure 4. Ionization profile due to the HECR component (above $1 \mathrm{GeV}$ ) under Galactic conditions and the LECR component (the possible range is shaded, considering the minimum W98 and maximum M02 LECR spectra), as labeled in the top panel-but shown in both panels. The top panel compares our result with other models: (1) P09, with their E00 CR spectrum; (2) Padovani et al. (2018), interstellar CR model (upper bound); (3) Silsbee \& Ivlev (2019) pure diffusion model, using same CR spectral model as Padovani et al. (2018) (lower bound); (4) P09, with their M02 spectral model; (5) Padovani et al (2018) interstellar CR model (lower bound); (6) P09, with their W98 spectral model; and (7) P09, with their C00 spectral model. The bottom panel compares our result with observed CR ionization rates in MCs, with data from Indriolo et al. (2015) and Neufeld \& Wolfire (2017) as labeled. The Neufeld \& Wolfire (2017) points are separated into measurements where column densities were obtained either from direct observations of $\mathrm{H}_{2}$ or were inferred indirectly from observations of $\mathrm{CH}$, or $E(B-V)$.

above a $\mathrm{GeV}$, is shown separately (as labeled) with rates of $\zeta^{\mathrm{H}}=0.6-1.1 \times 10^{-18} \mathrm{~s}^{-1}$, which are somewhat lower than the widely adopted values of $\zeta^{\mathrm{H}}=10^{-17}-10^{-15} \mathrm{~s}^{-1}$ in dense cores (Caselli et al. 1998; van der Tak \& van Dishoeck 2000; Doty et al. 2002), and $\zeta^{\mathrm{H}}=10^{-16} \mathrm{~s}^{-1}$ in the diffuse inter-clump medium (Black et al. 1978; van Dishoeck \& Black 1986; Federman et al. 1996). This difference can be attributed to the low-energy cosmic-ray (LECR) component, below a GeV, as indicated by the shaded region in Figure 4. Although their energy density is less than the HECRs, LECRs more strongly engage in ionization processes and so can elevate the rate to $\zeta^{\mathrm{H}}=10^{-17} \mathrm{~s}^{-1}$ when adopting the "minimum" LECR species spectra in the W98 (Webber 1998) and C00 (Strong et al. 2000) models, or $\zeta^{\mathrm{H}}=10^{-14} \mathrm{~s}^{-1}$ if adopting "maximum" spectra in the M02 (Moskalenko et al. 2002) and E00 (Strong et al. 2000) models. In the upper panel of Figure 4, we show a comparison between our model and ionization rates from other models in the literature. We note that the results of P09, shown by the lines labeled 1, 4, 6, and 7, adopt the same CR spectra as considered in this work, so differences can be attributed to the treatment of $\mathrm{CR}$ transport. The lower panel of Figure 4

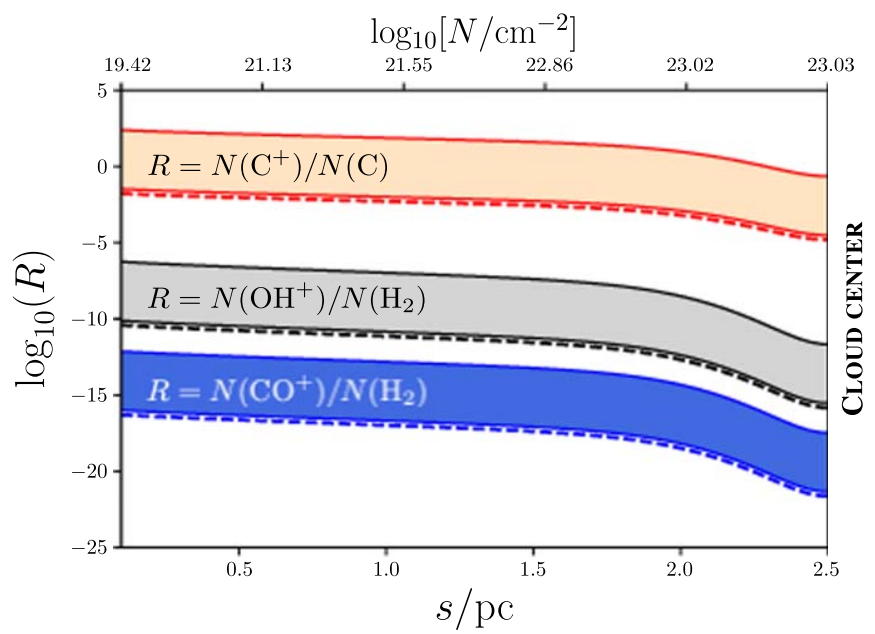

Figure 5. Abundance ratios due to $\mathrm{CR}$ ionization within the $\mathrm{MC}$ model, showing $N\left(\mathrm{C}^{+}\right) / N(\mathrm{C})$ (red), $N\left(\mathrm{CO}^{+}\right) / N\left(\mathrm{H}_{2}\right)$ (blue), and $N\left(\mathrm{OH}^{+}\right) / N\left(\mathrm{H}_{2}\right)$ (black). The shaded band represents the range of possible values from LECR ionizations only, while the single dashed line below each band represents the signature expected if only HECRs are present. Typically, any signal would therefore be dominated by the LECR ionizations.

additionally shows comparisons with recently observed CR ionization rates in MCs, which are broadly consistent with the results of this work.

These ionization profiles (again, accounting for the variation between the maximum and minimum LECR contribution to the ionization rates) can also be used to estimate abundance ratios for the species considered in Section 2.2.1, i.e., $N\left(\mathrm{CO}^{+}\right) / N\left(\mathrm{H}_{2}\right), N\left(\mathrm{OH}^{+}\right) / N\left(\mathrm{H}_{2}\right)$, and $N\left(\mathrm{C}^{+}\right) / N(\mathrm{C})$. We present the resulting profiles for the three ratios in Figure $5,{ }^{28}$ where the shaded ranges are due to LECR ionization and the dashed lines due to HECR ionization. The propagation of higher-energy CRs may differ substantially from their lower-energy counterparts due to, e.g., energy-dependent diffusion coefficients and the greater loss channels open to the higher-energy particles. It is therefore worthwhile to consider the signature of both components, even though the LECRs would usually dominate the signal, with the higher-energy ratios typically around an order of magnitude lower. While a single ratio should not be used independently to infer $\mathrm{CR}$ ionization rates, we note that the ratio $N\left(\mathrm{C}^{+}\right) / N(\mathrm{C})$ (in red) is much less sensitive to the underlying $\mathrm{MC}$ model than both $N\left(\mathrm{CO}^{+}\right) / N\left(\mathrm{H}_{2}\right)$ (blue) and $N\left(\mathrm{OH}^{+}\right) / N\left(\mathrm{H}_{2}\right)$ (black). This would make it a less stringent quantity to constrain $\mathrm{CR}$ ionization rates alone, and less powerful to diagnose the presence of CRs if alternative ratios are available. Indeed, this is in addition to possible contamination issues the $N\left(\mathrm{C}^{+}\right) / N(\mathrm{C})$ ratio would be susceptible to from any intervening non-shielded regions in the vicinity of the cloud, which would boost the $\mathrm{C}^{+}$abundance far above the levels driven by $\mathrm{CR}$ ionization processes.

Despite being more effective ionizers, the amount of energy available in LECRs to subsequently drive ionization-mediated heating effects is lower than in their higher-energy counterparts. Moreover, HECRs can heat a magnetized medium via Alfvénic mechanisms (see Section 2.2.2 for details), making them more effective than LECRs in this capacity. The specific heating rate due to HECRs in the MC is shown in Figure 6, where $\mathcal{H}$ is the sum of all heating contributions outlined in

\footnotetext{
${ }^{28}$ For reference, we find that our results for the $N\left(\mathrm{OH}^{+}\right) / N\left(\mathrm{H}_{2}\right)$ abundance ratio is broadly consistent with those presented in Hollenbach et al. (2012).
} 


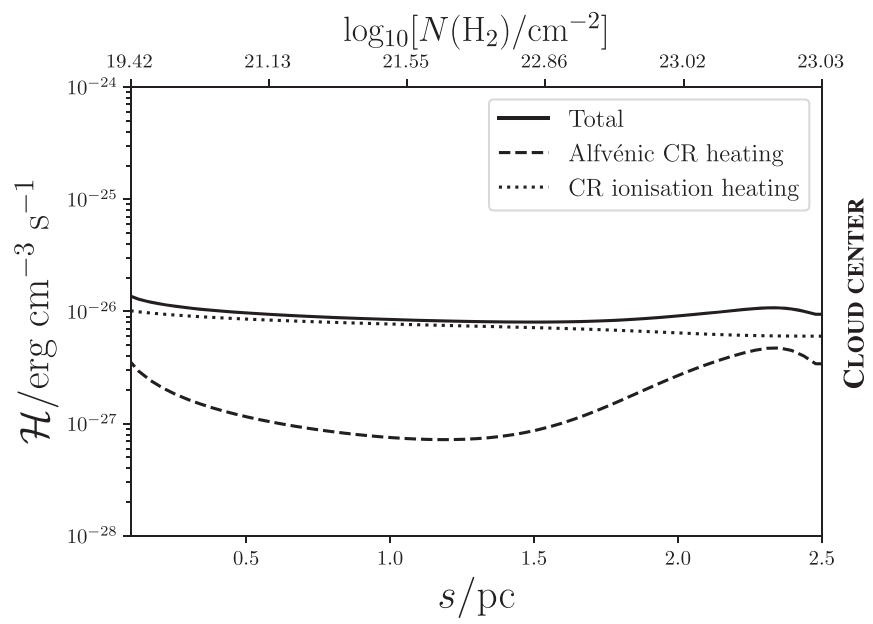

Figure 6. Total specific CR heating rates with contributions from Alfvénic and ionization heating in the idealized MC model under Galactic conditions. $\mathcal{H}$ is the sum of all CR heating contributions outlined in Section 2.2.2.

Section 2.2.2. Generally, ionization-driven heating dominates -however, in central regions the Alfvénic specific heating rate is boosted by the stronger magnetic field to become more competitive. The peak specific heating rate reaches around $10^{-26} \mathrm{erg} \mathrm{cm}^{-3} \mathrm{~s}^{-1}$ in the core, which is largely unchanged from the level experienced in the outer regions of the cloud. This value is slightly lower than less sophisticated estimates of $6.3 \times 10^{-27}\left(n_{\mathrm{H}} / 0.83 \mathrm{~cm}^{-3}\right) \mathrm{erg} \mathrm{cm}^{-3} \mathrm{~s}^{-1}$ (Spitzer \& Tomasko 1968; Goldsmith 2001), but around one order of magnitude higher than the estimated specific CR heating rate in the warm ionized medium outside the cloud (Wiener et al. 2013b). If the CR energy density is increased, the corresponding specific heating rates are boosted proportionally (see Figure 7). This indicates that the CR impact on the evolution of MCs could be strongly influenced by their surrounding conditions, e.g., due to the impact of a nearby SN event (see also Gabici et al. 2009), or if located in a star-forming or high-redshift galaxy (see Section 5).

By balancing CR heating rates against a simple cooling function (see Section 2.2.3), an equilibrium temperature can be estimated under different levels of CR irradiation. Figure 8 suggests that, in a Galactic scenario, the effect of an enhanced CR energy density would be minimal in the core regions of an MC where the temperature is only boosted marginally compared to the surrounding clump. Moreover, the core region only experiences modest increases in its equilibrium temperature, even when faced with substantial enhancements of the CR flux. Instead, the surrounding clump and inter-clump medium are more strongly impacted. The core temperatures estimated here are somewhat lower than expected, given that Galactic observations indicate core temperatures of around 8-12 K (Bergin \& Tafalla 2007). However, some starless cores are found to have temperatures of $T<7 \mathrm{~K}$ (Pagani et al. 2007, 2009, 2015; Lin et al. 2020), while theoretical studies have also found temperatures comparable to those calculated here in idealized cases (e.g., Juvela \& Ysard 2011). Although our results are consistent with these certain extreme cases (where core temperatures were argued to be as low as $6 \mathrm{~K}$ or even less, e.g., Harju et al. 2008), they suggest that either our adopted cooling function is overstated, or other processes, e.g., turbulent heating (Pan \& Padoan 2009), residual unattenuated interstellar radiation fields, and/or dust reprocessing/heating

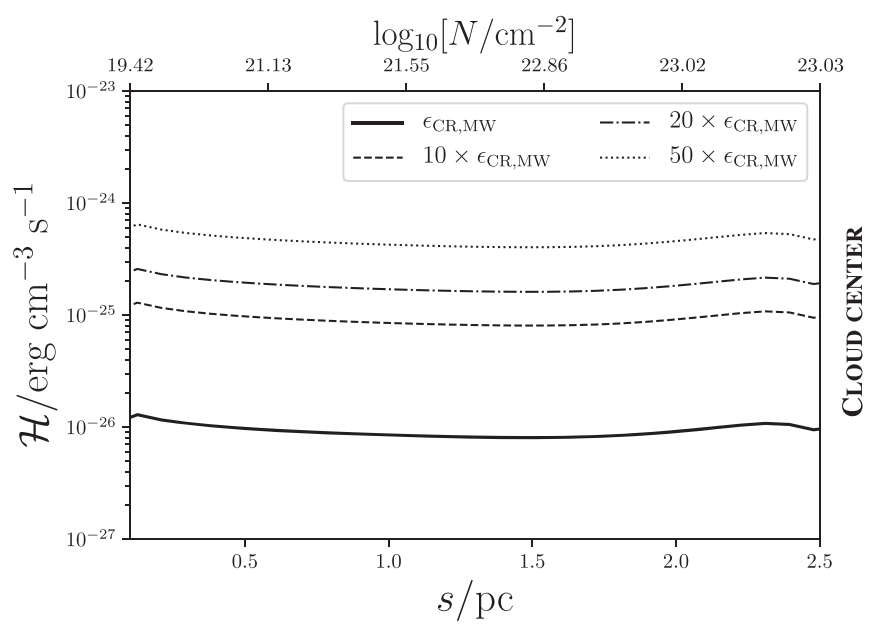

Figure 7. Total specific $\mathrm{CR}$ heating rates in the cloud under increasing ambient CR energy densities (as labeled), demonstrating the impact of different environments on the cloud's thermal evolution, even when shielded from the interstellar radiation field. The baseline case adopts the Milky Way CR energy density $\left(\epsilon_{\mathrm{CR}, \mathrm{MW}}\right)$, which is then scaled by the factors indicated. $\mathcal{H}$ is the sum of all CR heating contributions outlined in Section 2.2.2.

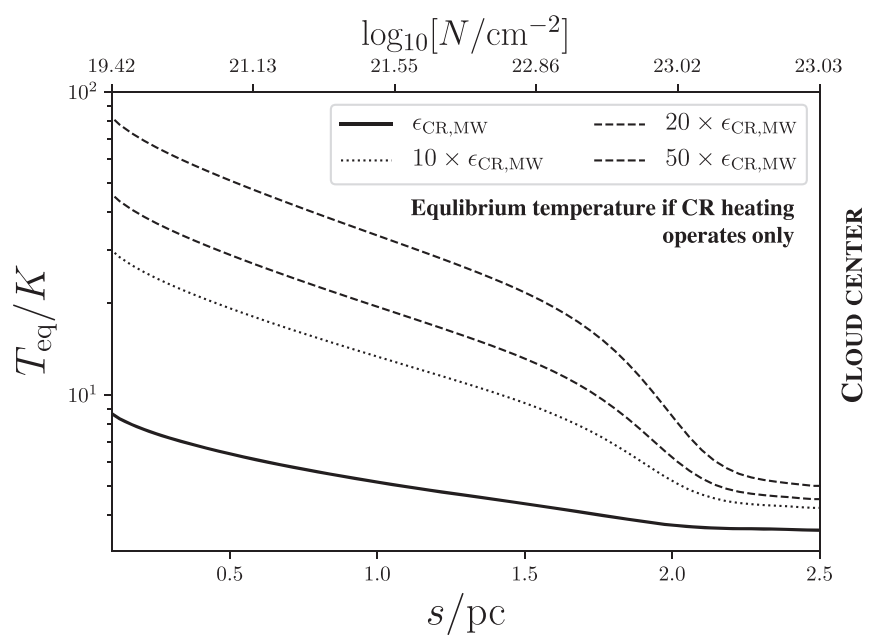

Figure 8. Equilibrium temperature profile arising from $\mathrm{CR}$ heating balanced against gas cooling under Galactic conditions, and with CR energy densities boosted by factors of 10,20 , and 50 . Other processes may operate to maintain higher temperatures of around $10 \mathrm{~K}$ observed in most Galactic MCs.

(Goldsmith 2001), may be operating alongside CRs to maintain higher core temperatures. If other heating mechanisms operate in competition with CRs, CR heating would only dominate the regulation of $\mathrm{MC}$ core temperatures in environments where the CR energy density is substantially greater than that typically estimated in the Galaxy. A more thorough assessment of the competition between various heating effects throughout MC subregions merits a dedicated study and is left to future work.

\subsection{Illustrative Case}

\subsubsection{The IC 5146 Cloud Region and Data Set}

The IC 5146 MC complex is located in Cygnus, exhibiting a converging filamentary system of dark clouds and elongated substructures extending from a main filament as seen with Herschel observations (Arzoumanian et al. 2011). Throughout the cloud, structures are in various stages of their evolution: the 


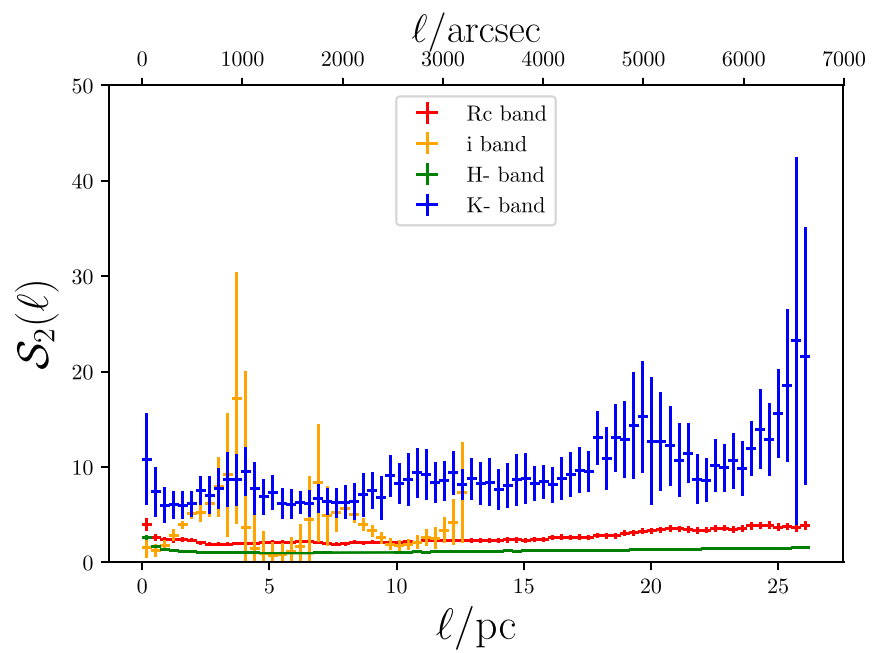

Figure 9. Dispersion functions calculated for the four bands. The increasing power at larger scales due to the curved hourglass magnetic field structure is not shown here as it is not relevant to our fluctuation analysis. $x$ error bars indicate the bin size. $y$ error bars are $1 \sigma$ Gaussian errors estimated by a Monte Carlo approach with 10,000 perturbations. A distance of 813 pc to IC 5146 is assumed (see the main text for details).

main filament and nearby Cocoon Nebula appears to be at different stages of ongoing star formation episodes (Harvey et al. 2008; Dunham et al. 2015), while some other dark cloud regions remain quiescent (Arzoumanian et al. 2011). The formation of the system is believed to be driven by large-scale turbulence (Arzoumanian et al. 2011), which would have introduced perturbations into the otherwise well-ordered largescale magnetic field morphology (Wang et al. 2017, 2019), thus making it an ideal test case for us to apply our model.

We note that there is some debate over the distance to the system (see, e.g., Lada et al. 1999; Harvey et al. 2008), and whether it is one system or two clouds along the same line of sight at different distances (Wang et al. 2020). Such matters would impact the conversion between angular separations and physical distances in this work. A recent reanalysis (if assuming it to be a single cloud) using Gaia DR2 data (Gaia Collaboration et al. 2018) indicates it to be located $813 \pm 106 \mathrm{pc}$ away (Dzib et al. 2018). We adopt this distance in our calculations, as in Wang et al. (2019).

For our analysis, we use optical and near IR stellar polarization observations toward IC 5146 (Wang et al. 2017) to trace the magnetic fields and its underlying fluctuations. The data set was compiled by matching polarization data to the positions of 2022 independent background stars to within $0.5^{\prime \prime}$ (corresponding to $0.002 \mathrm{pc}$ at a distance of $813 \mathrm{pc}$ ) from the Two Micron All-Sky Survey (Skrutskie et al. 2006) in at least one of the $R c, i^{\prime}, H$, and $K$ bands. In total, only three stars were present in all four bands, with around $71 \%$ of the stars being detected in the $H$ band, $24 \%$ in the $R c$ band, $10 \%$ in the $i^{\prime}$ band, and $8 \%$ in the $K$ band (Wang et al. 2017).

To estimate the diffusion coefficient of CRs through the region, we compute the angular dispersion function in every band using a bin size of $90^{\prime \prime}$ (corresponding to a physical size of $0.35 \mathrm{pc}$ ) which gives reasonable signal-to-noise ratios over the length scales of interest. Uncertainties are estimated using 10,000 Gaussian Monte Carlo perturbations to the Stokes parameters $Q$ and $U$ (see Figure 9, where $1 \sigma$ errors bars are shown). From our assessment of the distribution of separations of observed points, which do not reflect the features seen in
Figure 9, we argue that the structures evident in our dispersion analysis are likely to be physical in origin rather than due to instrumental or sampling effects. The FT of the dispersion function is calculated, and the diffusion coefficient for the region follows from Equation (42). We find that any spatial variation of the diffusion parameter $D$ arises from variations in the magnetic field strength alone, while spatial variations due to the field structure are not significant ${ }^{29}$ (see Appendix D for details). These findings are applied to our subsequent analysis of IC 5146, where we adopt an average value for the structural contribution $\mathcal{J}$ to the diffusion parameter (see Equation (38)) across the region, weighted by the number of background stars in each of the bands of the data set $\left(R c, i^{\prime}, H\right.$, and $\left.K\right)$. We find some variation of the diffusion coefficient, between $10^{29}$ and $10^{30} \mathrm{~cm}^{2} \mathrm{~s}^{-1}$ for a $1 \mathrm{GeV} \mathrm{CR}$ and a magnetic field of reference strength $B_{\text {ref }}=2.46 \mu \mathrm{G}$ (see Section 4.2.2), depending on which band of the data set is used in the analysis (see Appendix D for details). Our range of values is somewhat larger than the diffusion coefficients found in other studies, e.g., $10^{29} \mathrm{~cm}^{2} \mathrm{~s}^{-1}$ in the Galactic Center ridge (Gabici 2011), or between $10^{25}$ and $10^{27} \mathrm{~cm}^{2} \mathrm{~s}^{-1}$ in the Sgr B2 giant MC (Protheroe et al. 2008; Dogiel et al. 2015). We believe this may result from some small-scale structure in the magnetic field being missed by our analysis, as would arise from limited/ unavailable PA measurements on certain (smaller) separation scales, and/or projection effects that could mask magnetic field structures along the line of sight.

\subsubsection{Representation of the Filamentary Structures}

The IC 5146 region consists of a network of filamentary structures. Arzoumanian et al. (2011) identified 27 such filaments, to which they fit a cylindrical density profile of the form

$$
n_{\mathrm{H}}(s)=n_{\mathrm{c}}\left\{1+\left(\frac{s}{R_{\text {flat }}}\right)^{2}\right\}^{-p / 2},
$$

with $n_{\mathrm{c}}$ being the density of the filament ridge and $R_{\mathrm{flat}}$ is the characteristic length scale of the flat innermost portion of the profile. Observationally, it is convenient to express this in terms of column density along a line of sight to a radial position $s$ within the projected filament

$$
N_{\mathrm{H}}(s)=\mathcal{P} n_{\mathrm{c}} R_{\text {flat }}\left\{1+\left(\frac{s}{R_{\text {flat }}}\right)^{2}\right\}^{-\frac{p-1}{2}},
$$

where $\mathcal{P} \propto 1 / \cos \psi$ for $\psi$ is the angle of the filament to the sky plane. The variation of this parameter due to the inclination of IC 5146 to the sky plane is small, within a factor of 2 if assuming random orientations, and less than many other sources of uncertainty in our model. As such, we assume this system is oriented at $\psi=0^{\circ}$ to the plane of the sky. The magnetic field permeating this cloud is observed to be arranged predominantly perpendicularly to the filament orientations on the sky plane (Wang et al. 2017, 2020), and the mean magnetic field strength across the filaments can be well described by a

\footnotetext{
29 There is also no compelling support for a significant variation in the empirical value of $D$ between the different wave bands.
} 
Table 1

Specific Rates of CR Heating $\mathcal{H}$ and Ionization $\zeta^{\mathrm{H}}$ in 15 of the 27 Filaments in the IC 5146 Region Identified by Arzoumanian et al. (2011), Where Filament ID Numbers Here Correspond to Those Used in That Paper

\begin{tabular}{|c|c|c|c|c|c|c|c|c|}
\hline ID & $p$ & $R_{\text {flat }} / \mathrm{pc}$ & $n_{\mathrm{c}} / 10^{4} \mathrm{~cm}^{-3}$ & $\mathcal{H} / 10^{-26} \mathrm{erg} \mathrm{cm}^{-3} \mathrm{~s}^{-1}$ & $\overline{\zeta_{\text {LECRs }}^{\mathrm{H}, \min }} / 10^{-20} \mathrm{~s}^{-1}$ & $\overline{\zeta_{\text {LECRs }}^{\mathrm{H}, \max }} / 10^{-15} \mathrm{~s}^{-1}$ & 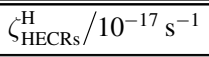 & $T_{\text {eq, }, \mathrm{CR}} / \mathrm{K}$ \\
\hline 1 & 2.1 & 0.09 & 0.3 & 0.59 & 2.1 & 4.4 & 1.1 & 0.8 \\
\hline 2 & 1.9 & 0.1 & 0.7 & 9.2 & 2.1 & 4.5 & 3.1 & 1.7 \\
\hline 4 & 1.4 & 0.04 & 0.7 & 4.3 & 2.1 & 4.5 & 1.9 & 1.3 \\
\hline 6 & 1.7 & 0.07 & 4 & 290 & 2.1 & 4.5 & 14 & 4.0 \\
\hline 7 & 1.6 & 0.05 & 2 & 33 & 2.1 & 4.5 & 5.6 & 2.2 \\
\hline 8 & 1.5 & 0.09 & 0.4 & 6.8 & 2.1 & 4.6 & 2.0 & 1.8 \\
\hline 11 & 1.9 & 0.07 & 1 & 6.5 & 2.1 & 4.4 & 3.2 & 1.5 \\
\hline 12 & 1.5 & 0.05 & 4 & 240 & 2.1 & 4.5 & 12 & 3.7 \\
\hline 13 & 1.6 & 0.04 & 3 & 4.3 & 2.1 & 4.4 & 6.8 & 2.3 \\
\hline 20 & 1.5 & 0.05 & 0.2 & 0.34 & 2.1 & 4.5 & 0.66 & 0.7 \\
\hline 21 & 1.7 & 0.09 & 0.3 & 1.9 & 2.1 & 4.5 & 1.4 & 1.2 \\
\hline 25 & 1.5 & 0.05 & 0.7 & 4.9 & 2.1 & 4.5 & 2.2 & 1.4 \\
\hline
\end{tabular}

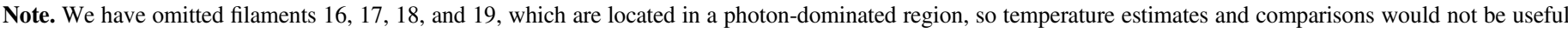

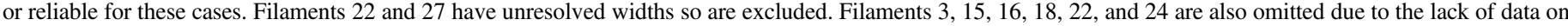

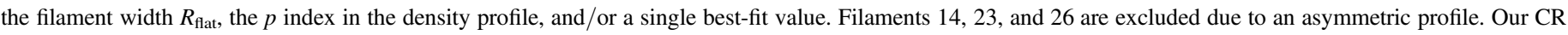

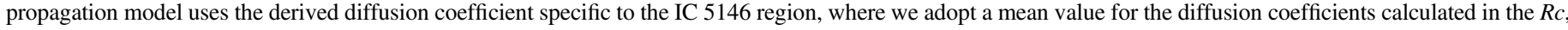

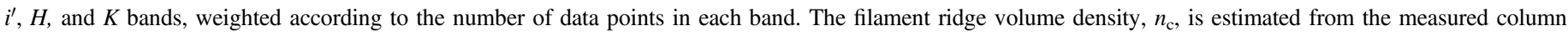

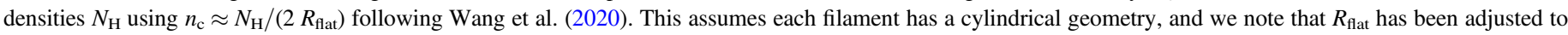

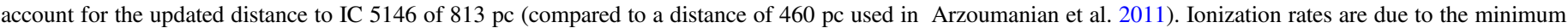

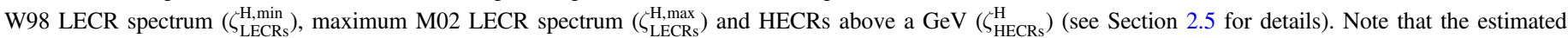

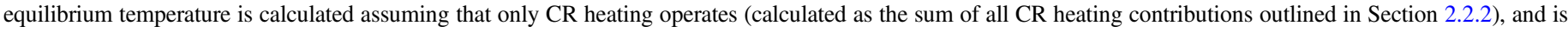

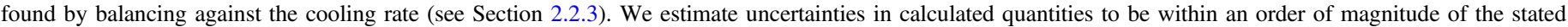
values, being dominated by the uncertainty in the empirical CR diffusion parameter.

simple power law,

$$
B_{\text {mean }}(n)=B_{0}\left(\frac{n}{150 \mathrm{~cm}^{-3}}\right)^{\bar{p}},
$$

with best-fit values of $B_{0}=2.46_{-0.51}^{+0.50} \mu \mathrm{G}$ and $\bar{p}=0.50_{-0.13}^{+0.12}$ (Wang et al. 2020). This prescription is specifically based on the analysis of IC 5146 and is different from our idealized case following Crutcher et al. (2010) (see Equation (49)). The filamentary structures are therefore ideal test cases of our model, where the heating and ionization rate profiles can be calculated through each filament by adopting a natural boundary condition at the filament edge. In this case, we take this edge to be at $\pm 1.5 \mathrm{pc}$ from the filament center, estimated from the proximity of the filaments in the region (Arzoumanian et al. 2011).

\subsubsection{Results}

Of the 27 distinct filamentary structures identified in Arzoumanian et al. (2011), there is sufficient parametric information (column density $N_{\mathrm{H}}$ and inner length scale $R_{\text {flat }}$ ) available to allow our model to be applied to 15 cases (see Table 1). For each case, we compute the ionization rate and specific heating rate, and deduce the equilibrium temperature. The resulting heating and ionization rates in the filament ridge (being their densest point) are shown in Table 1, together with an estimate of the equilibrium temperature that could be sustained if only CR heating processes were operating.

We have found that stronger heating typically occurs in the filaments that have larger volume densities. The effect is not linearly proportional to the density (e.g., compare filaments 6 and 7). This is because an increase in density will also lead to an increase in magnetic field strength inside a filament. This, in turn, increases the amount of deflection experienced by the CRs, but also the containment. The resulting impact of these antagonistic processes is determined by the extent of the cloud and the variation of the density profile, e.g., smaller values of $p$ yield a less steep magnetic profile and a relatively lower degree of mirroring to increase the heating efficiency. As shown, the strongest heating occurs in filaments 6 and 12. These have a high density but not a particularly steep density profile, thus reducing magnetic mirroring/deflection effects and allowing a substantial amount of CRs to penetrate inside. CR ionization rates due to both LECRs and HECRs are much less susceptible to density variations deep inside the cloud. This would imply relatively consistent $\mathrm{CR}$ ionization rates throughout Galactic MCs regardless of their internal configuration or exact filament/clump properties.

\subsection{Remarks}

A clear implication of our results in Table 1 is that CRdriven processes alone, while able to sustain relatively high rates of ionization within the cloud and heating rates comparable to estimates in the literature (see, e.g., Goldsmith 2001; Wiener et al. 2013b and Section 2.2.2), cannot maintain substantial core/filament temperatures if operating alone. Although caveats in our model would offer scope for larger values, this result taken by itself would suggest that other processes (e.g., heating from turbulence dissipation driven by gravitational collapse or other mechanical processes (see Carlberg \& Pudritz 1990; McKee \& Zweibel 1995; Gammie \& Ostriker 1996; Martin et al. 1997; Falceta-Gonçalves et al. 2003) may be more important in maintaining clump/filament temperatures at the expected level of around 8-12 K (Bergin \& Tafalla 2007) under Galactic conditions. This would point 
toward clump destruction being reliant on the emergence of protostars heating and ionizing dense regions from within, or photoevaporation/conduction from the external environment (i.e., outside-in destruction) rather than CR heating.

However, the models described in this work rely on a number of assumptions. Chief among these is the use of dust polarization of stellar radiation to trace the magnetic fields: the reliability of this indirect means of probing magnetic fields remains under debate-particularly toward the dense higher-extinction regions of principal interest in this work, and variation in alignment efficiency between individual sources (see Whittet et al. 2008; Cashman \& Clemens 2014) is well known. The observed PA dispersion is used to estimate the diffusion coefficient, however, in high density regions with poor radiative alignment efficiencies, the correspondence between dust alignment and magnetic field vectors could be weak-or timescales for radiative grain alignment to operate could become very long and unable to fully reflect rapidly varying turbulent magnetic field structures or fluctuations on very small scales. Moreover, CR heating of dust grains themselves (Kalvāns 2018) could also influence torque alignment efficiencies (see thermal wobbling in Lazarian \& Hoang 2007), and hence dust polarization. This would decrease the diffusion parameter and could substantially increase the CR heating rate felt in the densest regions. Future observations probing a wide range of magnetic field structures in MCs to higher resolution and avoiding the need to rely on dust alignment mechanisms may be possible with the upcoming Square Kilometer Array (see, e.g., Strong et al. 2014). Moreover, an assessment of the impact of various magnetic field configurations on the CR diffusion parameter and subsequent heating effect via simulations is worthy of a dedicated future study.

A further assumption is that the cooling is dominated by $\mathrm{CO}$ and dust (Goldsmith 2001; Galli et al. 2002), and we model this by invoking an analytical approximation (Goldsmith \& Langer 1978; Whitworth \& Jaffa 2018), which assumes dust temperatures of around $10 \mathrm{~K}$ (a compromise value between hotter regions in the cloud peripheries and cooler regions within the denser parts). While suitable under typical galactic MC conditions, variations in dust fraction, and/or composition may result in different cooling rates in alternative settings, e.g., in dusty galaxies or more/less chemically pristine or primordial environments (Jaacks et al. 2019). Moreover, substantial variation in dust and gas temperatures throughout a cloud would likely yield different cooling rates: presumably the rates adopted in this work are somewhat overstated in the densest core/filament regions where gas temperatures and subsequent cooling rates would be lower and less able to balance CR heating.

Variations in the irradiating CR flux or spectrum are also plausible. While this work has considered the effect of scaling the CR energy density and maintaining the same spectral form (see Figures 7 and 8), there is wide-ranging evidence in literature for different CR spectral indices at high energies in different environments - a harder spectrum in the vicinity of the Galactic ridge, where more CRs are freshly accelerated (Aharonian et al. 2006; Gaggero et al. 2017; H.E.S.S. Collaboration et al. 2018a, 2018b), would likely increase the CR heating rate.

\subsubsection{Implications}

Although relatively well shielded by the magnetic field configuration, the modest level of CR heating experienced in a filamentary clump or core would have substantial implications for

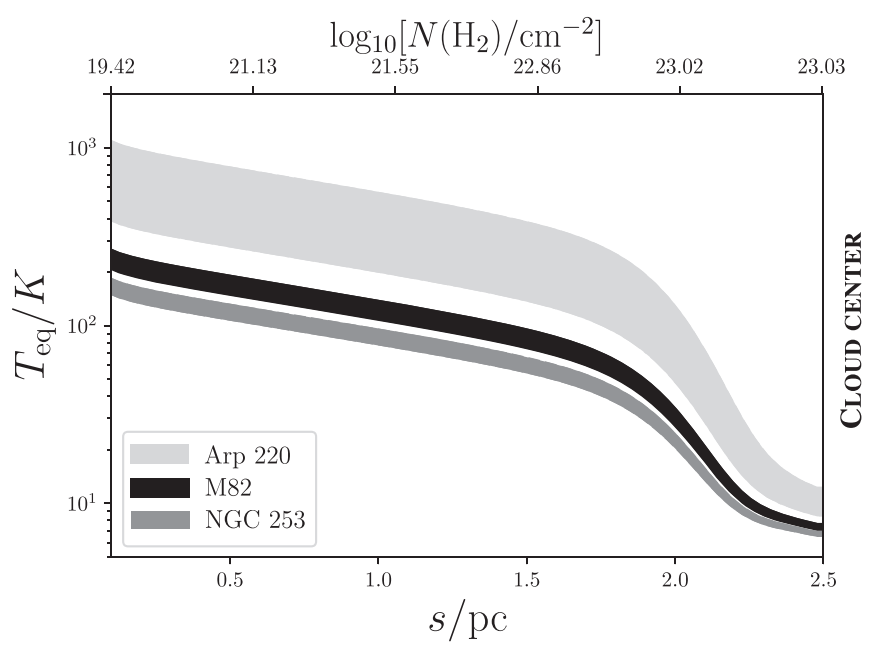

Figure 10. Equilibrium temperature accounting only for $C R$ heating when considering a cloud in different starburst environments. The CR energy density is estimated from Yoast-Hull et al. (2016), where we adopt the range calculated by $\gamma$-ray and radio emission. For Arp 220, we use the values associated with the surrounding torus region (see also Yoast-Hull et al. 2015) and assume a Galactic CR spectral shape.

subsequent star formation. Figure 10 explores the effect of different irradiating energy densities of CRs, scaled according to the estimated energy densities in nearby star-forming galaxies. In the exterior clump regions and inter-clump medium, a roughly linear relationship between CR energy density and resulting heating and equilibrium temperature is evident (see also Figures 7 and 8), but the effect on the core temperature is much more modest. Aside from magnetic mirroring/deflection effects, CR heating is much more important in the inter-clump (diffuse cloud) medium in general where the ionization fraction is higher and CRs are deflected less. This would favor clump heating via conduction rather than direct internal heating by the CRs.

Despite the core temperature increases remaining modest even when CR energy densities are enhanced substantially, the astrophysical implications can still be significant. The Jeans mass, for example, is dependent on temperature (as well as the volume density of gas, $n_{\mathrm{H}}$ ) as this sets the level of thermal pressure against gravitational collapse:

$$
M_{\mathrm{J}}=1.9 \times 10^{2}\left(\frac{T}{10 \mathrm{~K}}\right)^{3 / 2}\left(\frac{n_{\mathrm{H}}}{10^{3} \mathrm{~cm}^{-3}}\right)^{-1 / 2} M_{\odot}
$$

While this is a crude measure of the maximum stable mass against collapse, neglecting cloud/clump fragmentation, smallscale magnetic/turbulent support, and the microphysics of the stellar initial mass function, it can still give an idea of the size of MCs and the stellar clusters they develop into. Without CR heating, Galactic clouds could reach around $200 M_{\odot}$ before they become unstable and gravitationally collapse. However, with Arp 220 levels of CR irradiation (see Figure 10 for equilibrium temperatures resulting from $\mathrm{CR}$ heating in Arp 220, M82, and NGC 253-three nearby starburst galaxies), this could increase to around $4 \times 10^{3} M_{\odot}$. The mass distribution of clouds/clumps in systems with higher CR energy densities (e.g., in star-forming galaxies) can therefore become distorted, as higher star formation rates would yield larger interstellar cloud sizes. This may favor more stochastic burst-like starforming episodes. More importantly, the larger mass threshold 
required for gravitational collapse would likely lead to a period of quenching in a galaxy rich in CRs, as it would take longer for sufficiently large MCs to gain enough mass to begin their collapse.

\section{Summary and Conclusions}

We investigate the heating effect of CRs inside MCs and the dense clumps within. We determine how CRs propagate and deposit their energy inside the MCs using a CR transport formulation for a model specified by density and magnetic field profiles. We find that for an irradiating CR flux comparable to in the Milky Way, the specific heating rate can reach a level of $\sim 10^{-26} \mathrm{erg} \mathrm{cm}^{-3} \mathrm{~s}^{-1}$ in the densest regions of the cloud. This heating is somewhat suppressed by magnetic deflection effects, but comparable to the surrounding inter-clump medium. We also investigate the ionization caused by $\mathrm{CRs}$ and find observed rates in the range of $\zeta^{\mathrm{H}}=10^{-17}-10^{-15} \mathrm{~s}^{-1}$ (Black et al. 1978; van der Tak \& van Dishoeck 2000; Doty et al. 2002) can be reproduced by our model, with ionization being dominated by the contribution from LECRs below a GeV. Higher-energy CRs are more engaged with heating processes.

We further apply our model to the IC 5146 star-forming region in Cygnus, where we use observed dust PAs to estimate the $\mathrm{CR}$ diffusion coefficient through the region due to fluctuations in the local magnetic field (which we assume to be traced by the dust grain alignment). We calculate the CR heating and ionization rates in 15 of the filamentary structures identified in IC 5146, and find a broad variation of specific heating rates despite ionization rates remaining quite uniform between different filaments. While a specific CR heating rate ranging from $10^{-27}-10^{-24} \mathrm{erg} \mathrm{cm}^{-3} \mathrm{~s}^{-1}$ has not been shown to lead to high equilibrium temperatures of the filaments, MCs in environments with a strong CR flux (e.g., in star-forming galaxies) can be heated sufficiently, resulting in an increase of the Jeans mass. This would favor larger ISM clump sizes and presumably would yield a greater tendency for stochastic burstlike star formation histories to emerge. Moreover, it could also arguably lead to periods of quenching.

This work used high-performance computing facilities at the Center for Informatics and Computation in Astronomy (CICA), operated by the National Tsing Hua University (NTHU) Institute of Astronomy. This equipment was funded by the Taiwan Ministry of Education and the Taiwan Ministry of Science and Technology. E.R.O. and A.Y.L.O. are supported by CICA at NTHU through a grant from the Ministry of Education of the Republic of China (Taiwan). E.R.O.'s visits to NTHU were supported by the NTHU International Exchange Scholarship, hosted by S.P.L., and by the Ministry of Science and Technology of the Republic of China (Taiwan) grants 105-2119-M-007-028MY3 and 107-2628-M-007-003, hosted by Prof. Albert Kong. A.Y.L.O.'s visit to NTHU was supported by the Ministry of Science and Technology of the Republic of China (Taiwan) grant 105-2119-M-007-028-MY3, hosted by Prof. Albert Kong. We thank Sheng-Jun Lin (NTHU) for carefully reading through the manuscript. We also thank Dr. Jia-Wei Wang (Academica Sinica Institute for Astronomy and Astrophysics), Sheng-Jun Lin and Hao-Yuan Duan (NTHU), and Dr. Kate Pattle (NUI Galway) for discussions on MCs and the IC 5146 polarization data, and Dr. Ignacio Ferreras (Instituto de Astrofísica de Canarias) on the astrophysical implications of this work. E.R.O. and A.Y.L.O. also thank Dr. Curtis Saxton (University of Leeds) and Y.X. Jane
Yap (NTHU) for discussions and assistance with angular dispersion function (structure function) analyses. We are grateful to Prof. Vladimir Dogiel (PN Lebedev Institute of Physics), Prof. Chung-Ming Ko (National Central University) for their comments on this article, and the anonymous referees for their helpful and constructive feedback, which substantially improved the manuscript. This research has made use of NASA's Astrophysics Data System.

Software: SciPy (Virtanen et al. 2020), NumPy (Harris et al. 2020).

\section{Appendix A}

\section{Numerical Scheme for Solving the Transport Equation}

We introduce the variables $X(E, s)=n(E, s)$ and $U(s)=D$ $(E, s) \partial X / \partial s$, and the indices $q$ and $r$ for the energy $E_{\mathrm{q}}$ and spatial $s_{\mathrm{r}}$ grid points, respectively, when discretizing the partial differential Equations (29)-(31). We thus seek a numerical solution for $X_{\mathrm{q}, \mathrm{r}}$ in each case. Due to the large differences in the variations of each of the terms (especially in Appendices A.2 and A.3), the greater numerical stability afforded by an implicit Runge-Kutta (RK) 4/5 scheme was required in order to respond to the inherent "stiffness" of the problem. The RADAU5 implementation from Hairer et al. (1993) was adopted. ${ }^{30}$

\section{A.1. Primary Protons}

Equation (29) is discretized as follows, when rewritten as a system of two difference equations:

$$
\begin{gathered}
U_{\mathrm{q}, \mathrm{r}+1}=U_{\mathrm{q}, \mathrm{r}}+\left.\frac{\partial U}{\partial s}\right|_{\mathrm{q}, \mathrm{r}} \Delta s ; \\
X_{\mathrm{q}, \mathrm{r}+1}=X_{\mathrm{q}, \mathrm{r}}+\frac{U_{\mathrm{q}, \mathrm{r}}}{D\left(E_{\mathrm{q}}\right)} \Delta s,
\end{gathered}
$$

where $\Delta s$ is the step size in the $s$ direction into the MC. To solve them we consider a two-step scheme:

$$
\begin{gathered}
\left.\frac{\partial U}{\partial s}\right|_{\mathrm{q}, \mathrm{r}+1}=\left.\frac{\partial U}{\partial s}\right|_{\mathrm{q}, \mathrm{r}}+\Delta s\left\{\left.v_{\mathrm{A}}\left(s_{\mathrm{r}}\right) \frac{\partial X}{\partial s}\right|_{\mathrm{q}, \mathrm{r}}\right. \\
\left.+\frac{X_{\mathrm{q}+1, \mathrm{r}} b_{\mathrm{q}+1, \mathrm{r}}-X_{\mathrm{q}-1, \mathrm{r}} b_{\mathrm{q}-1, \mathrm{r}}}{2 \Delta E}+n_{\mathrm{H}}\left(s_{\mathrm{r}}\right) X_{\mathrm{q}, \mathrm{r}} \sigma_{\mathrm{p} \pi}\left(E_{\mathrm{q}}\right) c\right\} \\
\left.\frac{\partial X}{\partial s}\right|_{\mathrm{q}, \mathrm{r}}=\frac{U_{\mathrm{q}, \mathrm{r}}}{D\left(E_{\mathrm{q}}, s_{\mathrm{r}}\right)}
\end{gathered}
$$

The boundary conditions are

$$
\begin{gathered}
X_{1,1}=X_{1, r_{\max }}=n_{\mathrm{p}}\left(E_{1}, s_{1}\right) ; \\
U_{1,1}=U_{1, r_{\max }}=j\left(E_{1}, s_{1}\right),
\end{gathered}
$$

where $r_{\max }$ is the maximum index on the spatial grid (i.e., the upper boundary of the system), and the CR influx, $j$, is estimated from Padovani et al. (2009).

\section{A.2. Primary Electrons}

In this case, we solve Equation (30) using the same iterative scheme as set out in Equations (A1) and (A2), but with

\footnotetext{
${ }^{30}$ When parameter choices were selected to avoid stiffness issues, the explicit RK-Fehlberg implementation in Press et al. (1992) gave equivalent results.
} 
Equation (A3) replaced with

$$
\begin{aligned}
\left.\frac{\partial U}{\partial s}\right|_{\mathrm{q}, \mathrm{r}+1}= & \left.\frac{\partial U}{\partial s}\right|_{\mathrm{q}, \mathrm{r}}+\Delta s\left\{\left.v_{\mathrm{A}}\left(s_{\mathrm{r}}\right) \frac{\partial X}{\partial s}\right|_{\mathrm{q}, \mathrm{r}}\right. \\
& \left.+\frac{X_{\mathrm{q}+1, \mathrm{r}} b_{\mathrm{q}+1, \mathrm{r}}-X_{\mathrm{q}-1, \mathrm{r}} b_{\mathrm{q}-1, \mathrm{r}}}{2 \Delta E}\right\},
\end{aligned}
$$

and an appropriate choice of cooling function $b$. The boundary conditions are outlined in Section 2.4.4.

\section{A.3. Secondary Electrons}

The scheme for secondary electrons follows from the discretization of Equation (31). Again, the form is the same as in Appendix A.1, but where the inner Equation (A3) is replaced by

$$
\begin{aligned}
\left.\frac{\partial U}{\partial s}\right|_{\mathrm{q}, \mathrm{r}+1}= & \left.\frac{\partial U}{\partial s}\right|_{\mathrm{q}, \mathrm{r}}+\Delta s\left\{\left.v_{\mathrm{A}}\left(s_{\mathrm{r}}\right) \frac{\partial X}{\partial s}\right|_{\mathrm{q}, \mathrm{r}}\right. \\
& \left.+\frac{X_{\mathrm{q}+1, \mathrm{r}} b_{\mathrm{q}+1, \mathrm{r}}-X_{\mathrm{q}-1, \mathrm{r}} b_{\mathrm{q}-1, \mathrm{r}}}{2 \Delta E}-\left.Q_{\mathrm{e}}\right|_{\mathrm{q}, \mathrm{r}}\right\},
\end{aligned}
$$

with the appropriate choice of cooling function $b$. This time, the boundary conditions on $X$ are $X_{1,1}=X_{1, r_{\max }}=0$, with those on $U$ following similarly as $U_{1,1}=U_{1, r_{\max }}=0$. The source term depends on the primary proton/electron solutions above, with the $\left.Q_{\mathrm{e}}\right|_{\mathrm{q}, \mathrm{r}}$ injection term following as per the prescription in Section 2.4.5 at each point of the discretized grid.

\section{Appendix B Spectral Evolution}

Cooling and absorption losses as CR electrons and protons propagate through a cloud are relatively minimal for most energies of interest in this work. This is particularly the case at high energies, where MHD scattering losses operate for protons (presumably at a rate comparable to their heating power (see Wiener et al. 2013b for estimates of the heating power by this process), while additionally radiative, free-free and Coulomb losses also arise for electrons (see Owen et al. 2018 for associated timescales and length scales for relevant processes and their energy dependence; see also Dermer \& Menon 2009; Owen et al. 2019b). However, at low energies, ionization losses are more severe (P09) and can lead to significant spectral evolution through the cloud. In Figure B1, we show the spectral evolution for the model MC described in Section 4.1. The spectrum shown at $s=0$ is the initial boundary spectrum entering the region of influence of the cloud (see Section 2.5). This first shows a moderate increase at all energies, which is then followed by a moderate decrease deeper into the cloud. The initial increase near the cloud surface is largely driven by magnetic containment arising from slower CR diffusion in the strengthening magnetic field, while the antagonistic deflective effect of magnetic mirroring deeper into the cloud accounts for the subsequent decrease in CR density. The spectral evolution at lower energies (see Figure B2, which replots the sub-GeV component of the spectra in Figure B1 for clarity; note that the proton spectrum is multiplied by $E_{\mathrm{p}}^{-0.95}$ in the top panel to better show the spectral change) is driven by ionization losses. This exhibits a similar energy dependence for both protons and electrons (see the adopted ionization cross
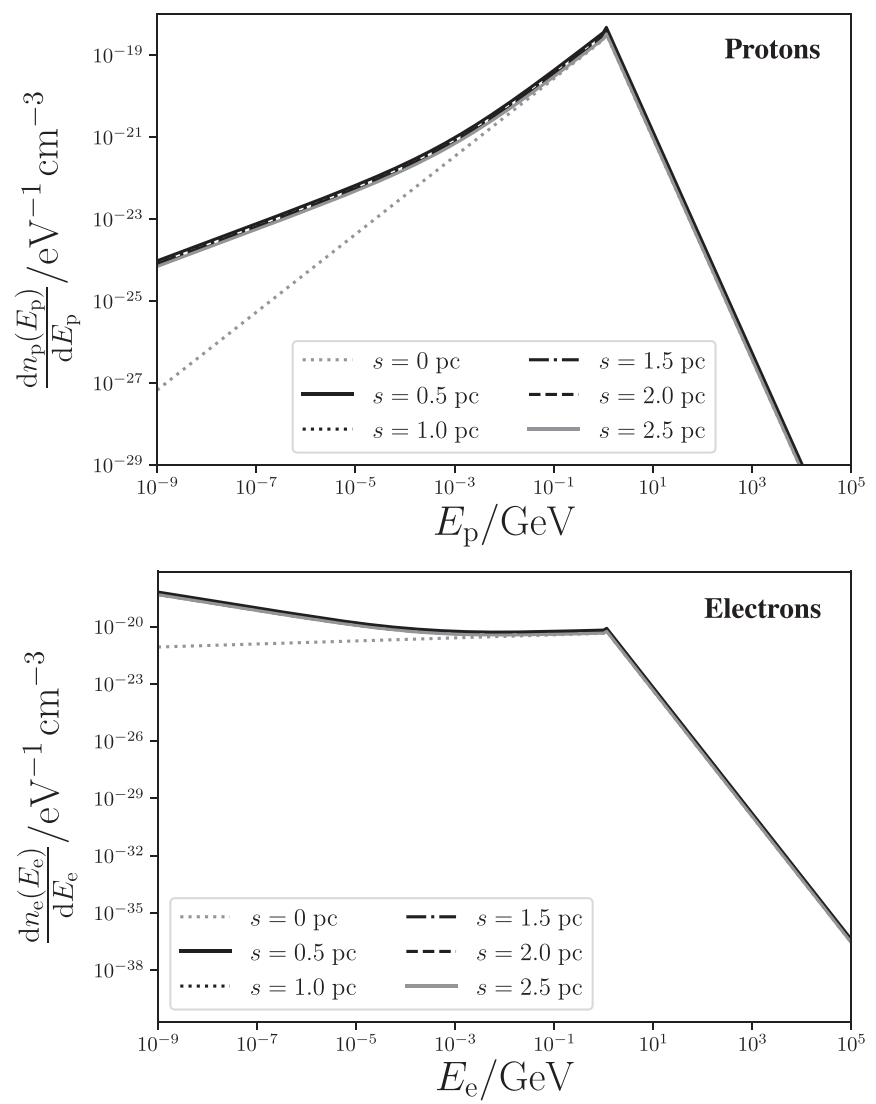

Figure B1. Proton and (primary) electron CR spectra at different positions within the cloud, with $s=0 \mathrm{pc}$ at the boundary corresponding to a column density of $2.6 \times 10^{19} \mathrm{~cm}^{-2}$ (and shows the adopted boundary spectrum (see Section 2.5), then at distances (column densities) of $s=0.5 \mathrm{pc}$ $\left(1.3 \times 10^{21} \mathrm{~cm}^{-2}\right), 1.0 \mathrm{pc}\left(3.5 \times 10^{21} \mathrm{~cm}^{-2}\right), 1.5 \mathrm{pc}\left(7.2 \times 10^{22} \mathrm{~cm}^{-2}\right), 2.0$ pc $\left(1.0 \times 10^{23} \mathrm{~cm}^{-2}\right)$, and $2.5 \mathrm{pc}\left(1.1 \times 10^{23} \mathrm{~cm}^{-2}\right)$. The spectral evolution of the high-energy component of both spectra is insignificant, as seen by the effective preservation of the spectral shape deep into the cloud. Losses due to ionizations become severe at lower energies, and exhibit a similar energy dependence for both protons and electrons. This leads to the deformation of both low energy spectra.

sections in P09). This is countered, and ultimately overcome, by the mirroring effects that reduce the $\mathrm{CR}$ density-but the decrease due to mirroring is noticeably moderated at these lower energies, which gain from the CR ionization losses. Although not shown in Figure B2, a linear plot with mirroring effects removed indicates that the low energy CR density is increased by a comparable amount in each spatial increment (of order $10^{-25} \mathrm{eV}^{-1} \mathrm{~cm}^{-3}$ for protons, or $10^{-19} \mathrm{eV}^{-1} \mathrm{~cm}^{-3}$ for electrons). This is in line with estimations: the ionization cross section peaks at around $\sigma^{\text {ion }} \sim 10^{-16} \mathrm{~cm}^{2}$ for both protons and electrons (P09). In a medium of density $n_{\mathrm{H}}=10 \mathrm{~cm}^{-3}$, this corresponds to an interaction rate of $R_{\text {ion }} \approx v \sigma_{\text {ion }} n_{\mathrm{H}}$, for $v$ as the CR effective macroscopic velocity. The time taken for $\mathrm{CRs}$ to propagate over a distance of $\ell_{\text {step }}=0.5 \mathrm{pc}$ (i.e., the distance increments in Figures B1 and B2) is then $t_{\text {step }} \sim \ell_{\text {step }} / v$. The number of ionizing interactions experienced by a CR beam in this interval then follows as approximately $t_{\text {step }} R_{\text {ion }} \sim \ell_{\text {step }} \sigma^{\text {ion }} n_{\mathrm{H}} \sim 10^{3}$. Thus for $\mathrm{CR}$ electrons (protons), of number density initially around $10^{-22} \mathrm{eV}^{-1} \mathrm{~cm}^{-3}\left(10^{-28} \mathrm{eV}^{-1} \mathrm{~cm}^{-3}\right)$ there would be around $10^{3}$ ionizing events in a $0.5 \mathrm{pc}$ interval, moving this number of particles from higher-energy bands to lower energies, where (without mirroring effects) they would increase 

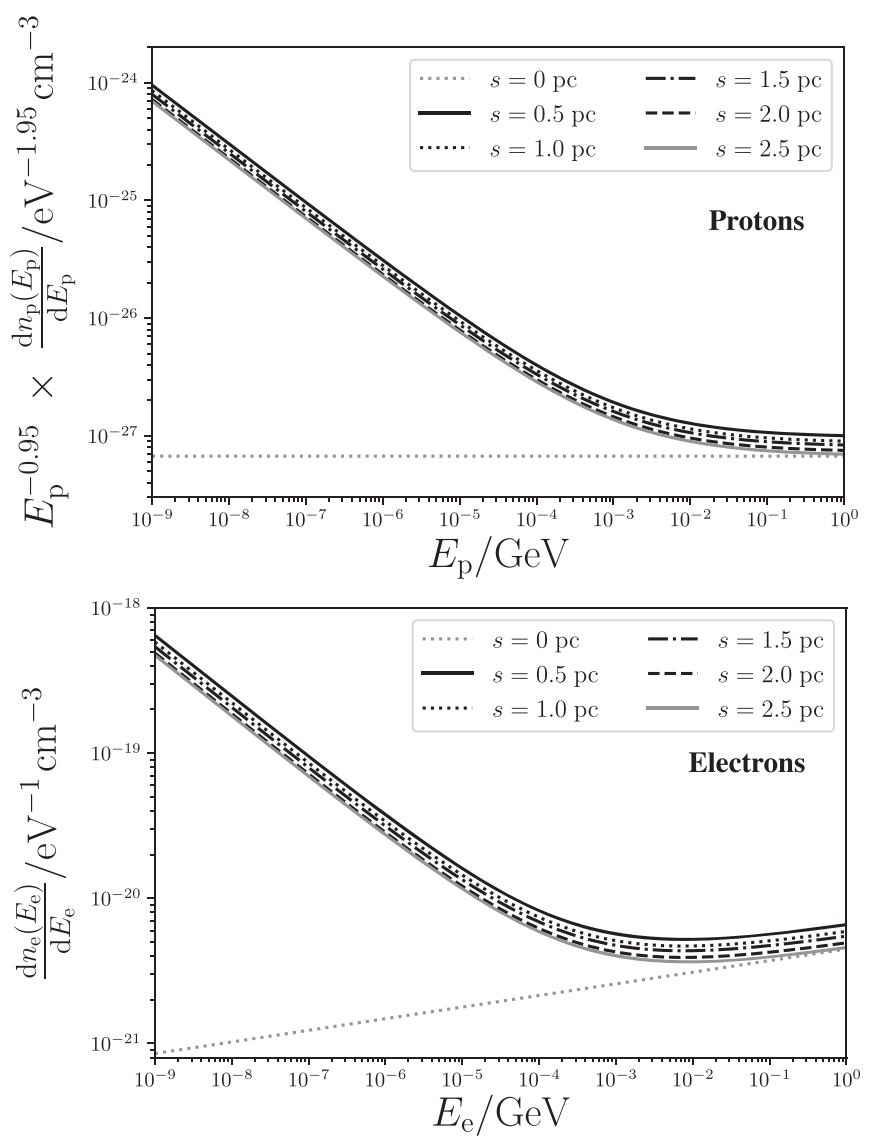

Figure B2. Same as Figure B1, but showing energies below $1 \mathrm{GeV}$ only. Note that the proton spectrum is multiplied by $E_{\mathrm{p}}^{-0.95}$ to more clearly show the spectral evolution.

the number density by around $10^{-19} \mathrm{eV}^{-1} \mathrm{~cm}^{-3}$ for electrons $\left(10^{-25} \mathrm{eV}^{-1} \mathrm{~cm}^{-3}\right.$ for protons).

\section{Appendix C \\ Angular Dispersion Function and Power Spectrum}

We compute the empirical CR diffusion parameter from the power spectrum $\hat{P}(k)$ of the magnetic fields permeating an MC (see Section 3.2.2). CR diffusion is determined by the properties and structures of the magnetic field. If the effects due to magnetic field dominate, we can compute the dispersion function $\mathcal{S}_{2}(\ell)$ from the magnetic field power spectrum $\hat{P}(k)$ under a slab approximation, i.e., where it only depends on the parallel component of $\boldsymbol{k}$ (Hasselmann \& Wibberenz 1968). Therefore, $\hat{P}(k)$ can be related to the dispersion function $\mathcal{S}_{2}(\ell)$ (more generally referred to as the second order structure function) of observed PAs through the cloud via a Fourier transform, denoted here as $\mathcal{F}[\ldots]$. From the Wiener-Khinchin theorem (Wiener 1930; Percival \& Walden 1993),

$$
\hat{P}(k)=\mathcal{F}[\mathcal{A}(\ell)]=\int_{-\infty}^{\infty} d \ell \mathcal{A}(\ell) \exp (i k \ell),
$$

where the autocorrelation function $\mathcal{A}(\ell)$ in the case of a statistically homogeneous and isotropic field can be expressed in terms of $\mathcal{A}(\ell)=\mathcal{A}(0)-\mathcal{S}_{2}(\ell) / 2$ (e.g., Schulz-Dubois \&
Rehberg 1981). Therefore,

$$
\begin{aligned}
\hat{P}(k) & =\int_{-\infty}^{\infty} d \ell\left[\mathcal{A}(0)-\frac{1}{2} \mathcal{S}_{2}(\ell)\right] \exp (i k \ell) \\
& =\mathcal{A}(0) \delta(k)-\frac{1}{2} \mathcal{F}\left[\mathcal{S}_{2}(\ell)\right],
\end{aligned}
$$

where the first term on the right-hand side is unphysical when $k=0$ and vanishes when $k \neq 0$. Hence,

$$
\hat{P}(k)=\frac{1}{2} \mathcal{F}\left[\mathcal{S}_{2}(\ell)\right],
$$

since $\mathcal{S}_{2}(\ell)$ is real and the FT of $\mathcal{S}_{2}(\ell)$ has a Hermitian symmetry.

\section{Appendix D \\ Spatial Dependence of the Empirical Diffusion Parameter in IC 5146}

Our analysis in Section 3.2 assumes that the spatial dependence of the diffusion parameter is derived only from the spatial variation in the magnetic field strength, and we argue that there is no clear empirical evidence for variation of the diffusion parameter within the IC 5146 region due to the magnetic fluctuations. Here, we analyze four subregions of IC 5146 to demonstrate that there is no strong evidence to support large spatial variations in the diffusion parameter, if adopting a fixed magnetic field strength and gas density. We select four circular regions (of radius $10^{\prime}$, labeled $\mathrm{A}, \mathrm{B}, \mathrm{C}$, and $\mathrm{D}$ ) around the hub/core filament structures of the region, as shown in Arzoumanian et al. (2011) and Wang et al. (2017). We analyze the $R c, i^{\prime}, H$, and $K$ bands separately to determine whether there is any variation between observational bands, and indicate the number of data points in each region, summarized in Table D1.

The diffusion parameter is computed for each region and band according to Section 3.2.2, for which a magnetic field strength on the plane of the sky is estimated from the density using Equation (52) (a relation specifically for the IC 5146 region), and for a CR energy of $1 \mathrm{GeV}$. Given the large ranges in volume density throughout each region, we use a characteristic value of $10^{3} \mathrm{~cm}^{-3}$ for this comparative estimate and note that future dedicated work should more carefully quantify the structures of magnetic and density fields with higher resolution. For our current approach, this is sufficient as our analysis in Section 4.2 separately accounts for variation in density/magnetic fields through the IC 5146 filamentary structures. We present our comparative results in Figure D1, where error bars are at the $1 \sigma$ confidence level. There is a slight tension in the $R c$-band data between the values derived for the four regions, however, there is no evidence of variation in any of the other bands. Moreover, the $K$ band yields slightly lower values for the diffusion coefficient, which presumably results from each of the bands being sensitive to slightly different scales (see also Wang et al. 2019) and subjected to different opacities into the cloud. We argue there is insufficient evidence to motivate the use of region-specific computations of the diffusion coefficient, but the tension between the bands does merit further work and comparison with simulation results to assess the suitability of each observational band in estimating a local diffusion parameter. 

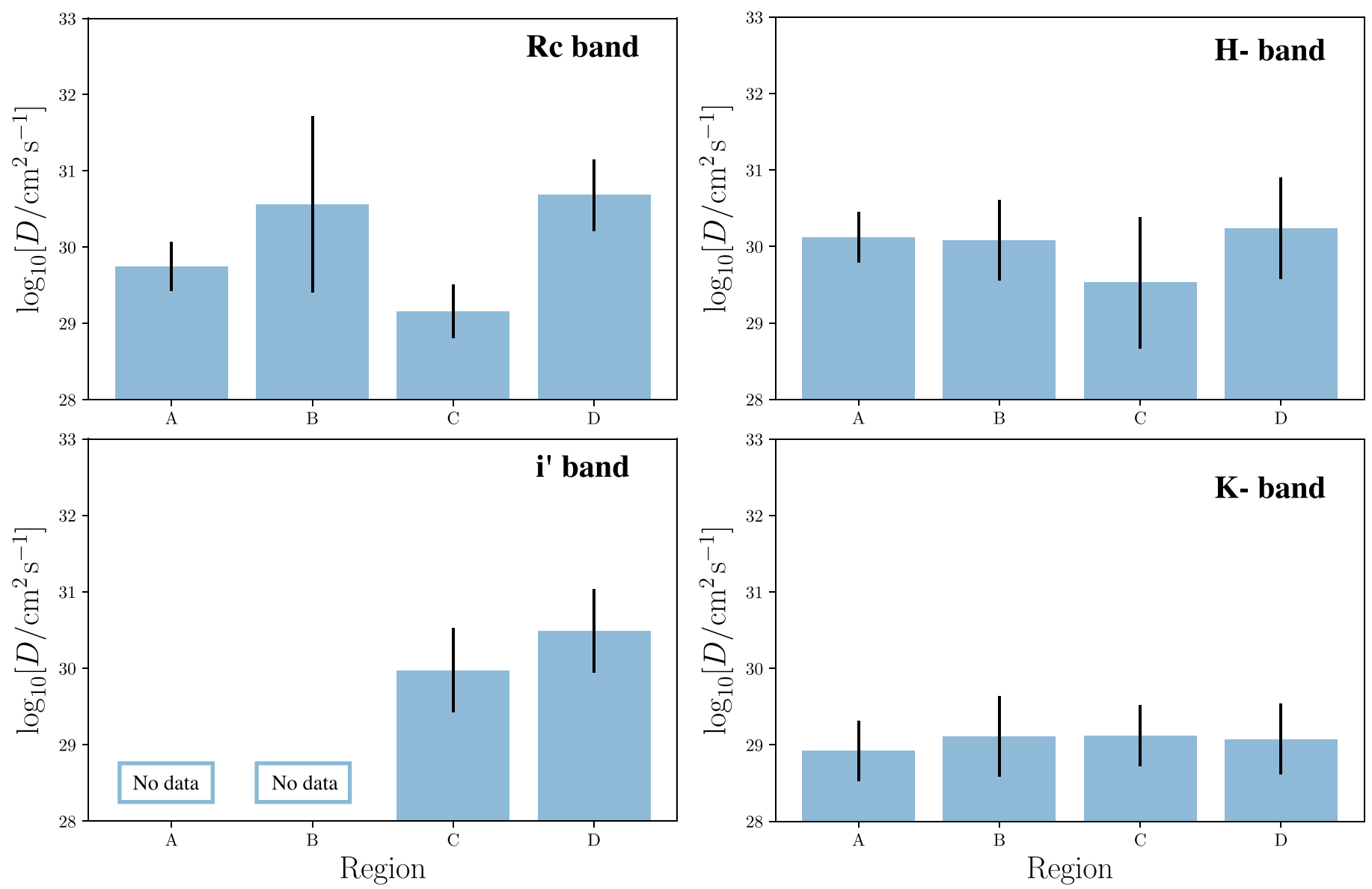

Figure D1. Estimated empirical diffusion parameter values for each of the regions A, B, C, and D in each of the four bands. $1 \sigma$ error bars are shown.

Table D1

Location and Number of Points in Each Analysis Region

\begin{tabular}{lccc}
\hline \hline Region & R.A. & Decl. & Number of Points \\
\hline A & $21^{\mathrm{h}} 53^{\mathrm{m}} 0^{\mathrm{s}}$ & $47^{\circ} 14^{\prime} 0^{\prime \prime}$ & $34(R c), 0\left(i^{\prime}\right), 120(H), 15(K)$ \\
B & $21^{\mathrm{h}} 50^{\mathrm{m}} 0^{\mathrm{s}}$ & $47^{\circ} 30^{\prime} 0^{\prime \prime}$ & $53(R c), 0\left(i^{\prime}\right), 87(H), 15(K)$ \\
C & $21^{\mathrm{h}} 45^{\mathrm{m}} 0^{\mathrm{s}}$ & $47^{\circ} 40^{\prime} 0^{\prime \prime}$ & $48(R c), 41\left(i^{\prime}\right), 165(H), 14(K)$ \\
D & $21^{\mathrm{h}} 48^{\mathrm{m}} 0^{\mathrm{s}}$ & $48^{\circ} 10^{\prime} 0^{\prime \prime}$ & $70(R c), 92\left(i^{\prime}\right), 187(H), 10(K)$ \\
\hline
\end{tabular}

Note. Each region selects all points within a radius of $10^{\prime}$ from the center. Note that only regions $\mathrm{C}$ and $\mathrm{D}$ intersect the smaller observation window for the $i^{\prime}$ band data. Region A roughly corresponds to the Cocoon Nebula.

\section{Appendix E Abundance Ratios}

CR ionization rates can be inferred from the astrochemistry of MCs. The derived rates account for direct CR ionizations together with the knock-on events caused by electrons being released in the ionization. The chemical processes can be split into three categories: (1) initiation steps arise when a species ionized by a CR subsequently reacts with species expected to be present in abundance in the $\mathrm{MC}$ environment (e.g., $\mathrm{H}, \mathrm{H}_{2}$, or C); (2) propagation steps yield the formation of readily observable species, or facilitate their destruction in a way which would impact on the chemical balance/abundance of that species, and (3) termination steps result in the neutralization of an ionized species within the chain, without leading to the production of a further species of interest - the "products" in Table E1, where the key reactions resulting from a CR ionization event in an MC are listed together with their associated rate coefficient. CR ionization in an MC proceeds as

$$
\begin{aligned}
\mathrm{H}+\mathrm{CR} & \rightarrow \mathrm{H}^{+}+\mathrm{CR}^{\prime} ; \\
\mathrm{H}_{2}+\mathrm{CR} & \rightarrow \mathrm{H}_{2}^{+}+\mathrm{CR}^{\prime} ; \\
\mathrm{C}+\mathrm{CR} & \rightarrow \mathrm{C}^{+}+\mathrm{CR}^{\prime},
\end{aligned}
$$

which lead directly to steps I1, I2, and I3, respectively (see ID keys in Table E1).

In a steady state the reactions in Table E1 give the abundance ratios for the species of interest, $\mathrm{OH}^{+}, \mathrm{CO}^{+}$, and $\mathrm{C}^{+}$(see Section 2.2.1). The key reactions yield

$$
\begin{gathered}
n\left(\mathrm{OH}^{+}\right)=\frac{n\left(\mathrm{O}^{+}\right) n\left(\mathrm{H}_{2}\right) k_{\mathrm{P} 1}+n\left(\mathrm{H}_{3}^{+}\right) n(\mathrm{O}) k_{\mathrm{P} 3}}{n\left(\mathrm{H}_{2}\right) k_{\mathrm{P} 4}+n_{\mathrm{e}} k_{\mathrm{T} 1}} ; \\
n\left(\mathrm{CO}^{+}\right)=\frac{n\left(\mathrm{C}^{+}\right) n(\mathrm{OH}) k_{\mathrm{I} 3}}{n(\mathrm{H}) k_{\mathrm{P} 5}+n\left(\mathrm{H}_{2}\right) k_{\mathrm{P} 6}+n_{\mathrm{e}} k_{\mathrm{T} 5}} ; \\
n\left(\mathrm{C}^{+}\right)=\frac{n(\mathrm{C}) \zeta^{\mathrm{H}}}{n(\mathrm{OH}) k_{\mathrm{I}}}
\end{gathered}
$$

which may be used together with

$$
n\left(\mathrm{H}_{3}^{+}\right)=\frac{n\left(\mathrm{H}_{2}\right) \zeta^{\mathrm{H}}}{n(\mathrm{CO}) k_{\mathrm{P} 2}+n(\mathrm{O}) k_{\mathrm{P} 3}+n_{\mathrm{e}}\left(k_{\mathrm{T} 2}+k_{\mathrm{T} 3}+k_{\mathrm{T} 4}\right)}
$$

to assess the chemical balances of the required species. The rate coefficients for the relevant processes are given in Table E1. 
Table E1

Rate Coefficients for Principal Formation/Destruction Reactions of Chemical Tracers in MCs as Collated in the UMIST RATE95 (Millar et al. 1997) and Updated UMIST RATE12 (McElroy et al. 2013) Databases

\begin{tabular}{lccc}
\hline \hline ID & Reaction & Rate Coefficient $^{\mathrm{a}}\left\{c_{1} / \mathrm{cm}^{3} \mathrm{~s}^{-1}, c_{2}, c_{3} / \mathrm{K}\right\}$ & Reference(s) $^{\mathrm{b}}$ \\
\hline $\mathrm{I} 1$ & $k_{\mathrm{I} 1}=\left\{7.0 \times 10^{-10}, 0.26,224.3\right\}$ & Stancil et al. (1999) \\
$\mathrm{I} 2$ & $k_{\mathrm{I} 2}=\left\{2.1 \times 10^{-9}, 0.0,0.0\right\}$ & Theard \& Huntress (1974) \\
$\mathrm{I} 3$ & $\mathrm{k}_{\mathrm{I} 3}=\left\{7.7 \times 10^{-10},-0.5,0.0\right\}$ & Prasad \& Huntress $(1980)$ \\
$\mathrm{P} 1$ & $k_{\mathrm{P} 1}=\left\{1.7 \times 10^{-9}, 0.0,0.0\right\}$ & Adams et al. (1980) \\
P2 & $\mathrm{H}_{2}^{+}+\mathrm{H}_{2} \rightarrow \mathrm{H}_{3}^{+}+\mathrm{H}$ & $k_{\mathrm{P} 2}=\left\{1.4 \times 10^{-9},-0.14,-3.4\right\}^{\mathrm{c}}$ & Klippenstein et al. (2010) \\
P3 & $\mathrm{C}^{+}+\mathrm{OH} \rightarrow \mathrm{CO}^{+}+\mathrm{H}$ & $k_{\mathrm{P} 3}=\left\{8.0 \times 10^{-10},-0.16,1.4\right\}$ & Bettens et al. (1999) \\
P4 & $\mathrm{O}^{+}+\mathrm{H}_{2} \rightarrow \mathrm{OH}^{+}+\mathrm{H}$ & $k_{\mathrm{P} 4}=\left\{1.0 \times 10^{-9}, 0.0,0.0\right\}$ & Jones et al. (1981) \\
P5 & $\mathrm{H}_{3}^{+}+\mathrm{CO} \rightarrow \mathrm{HCO}^{+}+\mathrm{H}_{2}$ & $k_{\mathrm{P} 5}=\left\{7.5 \times 10^{-10}, 0.0,0.0\right\}$ & Federer et al. (1984) \\
P6 & $\mathrm{H}_{3}^{+}+\mathrm{O} \rightarrow \mathrm{OH}^{+}+\mathrm{H}_{2}$ & $k_{\mathrm{P} 6}=\left\{1.8 \times 10^{-9}, 0.0,0.0\right\}$ & Adams et al. (1978) \\
T1 & $\mathrm{OH}^{+}+\mathrm{H}_{2} \rightarrow \mathrm{H}_{2} \mathrm{O}^{+}+\mathrm{H}$ & $k_{\mathrm{T} 1}=\left\{3.8 \times 10^{-8},-0.50,0.0\right\}$ & Mitchell (1990) \\
T2 & $\mathrm{CO}^{+}+\mathrm{H} \rightarrow \mathrm{CO}+\mathrm{H}^{+}$ & $k_{\mathrm{T} 2}=\left\{2.3 \times 10^{-8},-0.52,0.0\right\}$ & McCall et al. (2004) \\
T3 & $\mathrm{CO}^{+}+\mathrm{H}_{2} \rightarrow \mathrm{HCO}+\mathrm{H}$ & $k_{\mathrm{T} 3}=\left\{4.4 \times 10^{-8},-0.52,0.0\right\}$ & McCall et al. (2004) \\
T4 & $\mathrm{OH}^{+}+\mathrm{e}^{-} \rightarrow \mathrm{O}+\mathrm{H}$ & $k_{\mathrm{T} 4}=\left\{1.6 \times 10^{-8},-0.43,0.0\right\}$ & Mitchell (1990) \\
T5 & $\mathrm{H}_{3}^{+}+\mathrm{e}^{-} \rightarrow \mathrm{H}_{2}+\mathrm{H}$ & $k_{\mathrm{T} 5}=\left\{1.0 \times 10^{-7},-0.46,0.0\right\}$ & Mitchell (1990) \\
& $\mathrm{H}_{3}^{+}+\mathrm{e}^{-} \rightarrow 3 \mathrm{H}$ & &
\end{tabular}

Notes. Reaction IDs indicate initiation steps (I), propagation steps $(\mathrm{P})$, and termination steps $(\mathrm{T})$.

${ }^{a}$ Rate coefficients are given in terms of the parameters $\left\{c_{1}, c_{2}, c_{3}\right\}$, for $k_{\mathrm{xx}}=c_{1}(T / 300 \mathrm{~K})^{c_{2}} \exp \left(-c_{3} / T\right)$, where xx denotes the process ID.

${ }^{\mathrm{b}}$ Reference(s) of the original source of the rate coefficients in the RATE12 database (McElroy et al. 2013).

${ }^{\mathrm{c}}$ The sign of the parameter $c_{3}$ in process $\mathrm{P} 2$ differs from other processes due to the molecular geometry. The charge-dipole, charge-quadrupole, and the chargeinduced-dipole interactions influence the rate coefficient differently at different temperatures for the capture of reacting species in forming the transition state (see Klippenstein et al. 2010 for details).

${ }^{\mathrm{d}} \mathrm{CO}$ production via $\mathrm{CH}$ and $\mathrm{CH}_{2}$ channels can also become important when the metallicity is high (i.e., large $\mathrm{CH} / \mathrm{OH}$ ratio). We do not consider this channel in the reaction network as it is sub-dominant for the objects of interest in this work. This should, however, be included in the analyses of high metallicity (above solar) regions.

These assume that atomic and molecular hydrogen and atomic carbon ionization rates by $\mathrm{CRs}$ are all equivalent. Equation (E1) can be reduced to

$$
n\left(\mathrm{OH}^{+}\right) \approx \frac{\left[2+f_{\mathrm{H}_{2}}\right] \zeta^{\mathrm{H}}}{k_{\mathrm{P} 4} f_{\mathrm{H}_{2}}+2 x_{i} k_{\mathrm{T} 1}},
$$

assuming that the value of $n\left(\mathrm{H}_{3}^{+}\right) n(\mathrm{CO}) k_{\mathrm{P} 2}$ (i.e., the rate of process $\mathrm{P} 2$ ) and the $\mathrm{H}_{3}^{+}$dissociative recombination rate are negligible compared to the ionization rates of $\mathrm{H}$ and $\mathrm{H}_{2}$ (which would presumably be valid in a neutral $\mathrm{MC}$ with low ionization fraction). Moreover, Equation (E2) becomes

$$
n\left(\mathrm{CO}^{+}\right)=\frac{x_{\mathrm{C}} \zeta^{\mathrm{H}}}{\left(2 k_{\mathrm{P} 5} / f_{\mathrm{H}_{2}}\right)+k_{\mathrm{P} 6}+\left(2 x_{i} k_{\mathrm{T} 5} / f_{\mathrm{H}_{2}}\right)},
$$

where $x_{\mathrm{C}}=n(\mathrm{C}) / n\left(\mathrm{H}_{2}\right)$. These expressions, together with Equation (E3), then yield Equations (1)-(3) in Section 2.2.1, where ratios are taken to allow the abundances to be expressed as column densities.

\section{ORCID iDs}

Ellis R. Owen (iD https://orcid.org/0000-0003-1052-6439 Alvina Y. L. On (iD https://orcid.org/0000-0003-4479-4415 Shih-Ping Lai (i) https://orcid.org/0000-0001-5522-486X Kinwah Wu (i) https://orcid.org/0000-0002-7568-8765

\section{References}

Abraham, P. B., Brunstein, K. A., \& Cline, T. L. 1966, PhRv, 150, 1088 Adams, N. G., Smith, D., \& Grief, D. 1978, IJMSI, 26, 405

Adams, N. G., Smith, D., \& Paulson, J. F. 1980, JChPh, 72, 288

Aharonian, F., Bykov, A., Parizot, E., Ptuskin, V., \& Watson, A. 2012, SSRv, 166,97
Aharonian, F., Akhperjanian, A. G., Bazer-Bachi, A. R., et al. 2006, Natur, 439, 695

Albertsson, T., Kauffmann, J., \& Menten, K. M. 2018, ApJ, 868, 40

Almeida, S. P., Rushbrooke, J. G., Scharenguivel, J. H., et al. 1968, PhRv, 174,1638

Arzoumanian, D., André, P., Didelon, P., et al. 2011, A\&A, 529, L6

Axford, W. I., Leer, E., \& Skadron, G. 1977, Proc. ICRC (Budapest), 11, 132 Basu, S. 2000, ApJL, 540, L103

Basu, S., Ciolek, G. E., Dapp, W. B., \& Wurster, J. 2009, NewA, 14, 483

Bell, A. R. 1978a, MNRAS, 182, 147

Bell, A. R. 1978b, MNRAS, 182, 443

Berezinskii, V. S., Bulanov, S. V., Dogiel, V. A., \& Ptuskin, V. S. 1990, in Astrophysics of Cosmic Rays, ed. V. L. Ginzburg (Amsterdam: NorthHolland)

Bergin, E. A., \& Tafalla, M. 2007, ARA\&A, 45, 339

Berrington, R. C., \& Dermer, C. D. 2003, ApJ, 594, 709

Bettens, R. P. A., Hansen, T. A., \& Collins, M. A. 1999, JChPh, 111, 6322

Bisbas, T. G., van Dishoeck, E. F., Papadopoulos, P. P., et al. 2017, ApJ, 839,90

Black, J. H., Hartquist, T. W., \& Dalgarno, A. 1978, ApJ, 224, 448

Blandford, R. D., \& Ostriker, J. P. 1978, ApJL, 221, L29

Blasi, P. 2011, in Cosmic Rays for Particle and Astroparticle Physics, ed. S. Giani, C. Leroy, \& P. G. Rancoita (Singapore: World Scientific), 493

Blattnig, S. R., Swaminathan, S. R., Kruger, A. T., et al. 2000, Parameterized Cross Sections for Pion Production in Proton-Proton Collisions, Tech. Rep. NASA/TP-2000-210640, NASA

Blumenthal, G. R. 1970, PhRvD, 1, 1596

Brown, R. L., \& Marscher, A. P. 1977, ApJ, 212, 659

Brunstein, K. A. 1965, PhRv, 137, 757

Bykov, A. M., Marcowith, A., Amato, E., et al. 2020, SSRv, 216, 42

Carlberg, R. G., \& Pudritz, R. E. 1990, MNRAS, 247, 353

Casanova, S., Aharonian, F. A., Fukui, Y., et al. 2010, PASJ, 62, 769

Caselli, P., Walmsley, C. M., Terzieva, R., \& Herbst, E. 1998, ApJ, 499, 234

Cashman, L. R., \& Clemens, D. P. 2014, ApJ, 793, 126

Ceccarelli, C., Caux, E., Wolfire, M., et al. 1998, A\&A, 331, L17

Cécere, M., Velázquez, P. F., Araudo, A. T., et al. 2016, ApJ, 816, 64

Cesarsky, C. J., \& Volk, H. J. 1978, A\&A, 70, 367

Chandran, B. D. G. 2000, ApJ, 529, 513

Chandrasekhar, S., \& Fermi, E. 1953, ApJ, 118, 113

Chernyshov, D. O., Caselli, P., Cheng, K. S., et al. 2018, Nuclear and Particle Physics Proc., 297, 80 
Ching, T.-C., Lai, S.-P., Zhang, Q., et al. 2017, ApJ, 838, 121

Colafrancesco, S., \& Marchegiani, P. 2008, A\&A, 484, 51

Commerçon, B., Marcowith, A., \& Dubois, Y. 2019, A\&A, 622, A143

Cooley, J. W., \& Tukey, J. W. 1965, MaCom, 19, 297

Coudé, S., Bastien, P., Houde, M., et al. 2019, ApJ, 877, 88

Crutcher, R. M. 1999, ApJ, 520, 706

Crutcher, R. M. 2012, ARA\&A, 50, 29

Crutcher, R. M., Troland, T. H., Goodman, A. A., et al. 1993, ApJ, 407, 175

Crutcher, R. M., Wandelt, B., Heiles, C., Falgarone, E., \& Troland, T. H. 2010, ApJ, 725, 466

Cummings, A. C., Stone, E. C., Heikkila, B. C., et al. 2016, ApJ, 831, 18

Dalgarno, A. 2006, PNAS, 103, 12269

Dalgarno, A., \& McCray, R. A. 1972, ARA\&A, 10, 375

Davis, L. 1951, PhRv, 81, 890

Dermer, C. D., \& Menon, G. 2009, High Energy Radiation from Black Holes: Gamma Rays, Cosmic Rays, and Neutrinos (Princeton, NJ: Princeton Univ. Press)

Desch, S. J., Connolly, H. C., Jr., \& Srinivasan, G. 2004, ApJ, 602, 528

Dib, S., Shadmehri, M., Padoan, P., et al. 2010, MNRAS, 405, 401

Dogel, V. A., \& Sharov, G. S. 1990, A\&A, 229, 259

Dogiel, V. A., Chernyshov, D. O., Ivlev, A. V., et al. 2018, ApJ, 868, 114

Dogiel, V. A., Chernyshov, D. O., Kiselev, A. M., et al. 2015, ApJ, 809, 48

Dolginov, A. Z., \& Mitrofanov, I. G. 1976, Ap\&SS, 43, 291

Doty, S. D., van Dishoeck, E. F., van der Tak, F. F. S., \& Boonman, A. M. S. 2002, A\&A, 389, 446

Draine, B. T. 2011, Physics of the Interstellar and Intergalactic Medium (Princeton, NJ: Princeton Univ. Press)

Draine, B. T., \& Weingartner, J. C. 1996, ApJ, 470, 551

Draine, B. T., \& Weingartner, J. C. 1997, ApJ, 480, 633

Dunham, M. M., Allen, L. E., Evans, J. I., et al. 2015, ApJS, 220, 11

Dzib, S. A., Loinard, L., Ortiz-León, G. N., Rodríguez, L. F., \& Galli, P. A. B. 2018, ApJ, 867, 151

Elmegreen, B. G. 1979, ApJ, 232, 729

Falceta-Gonçalves, D., de Juli, M. C., \& Jatenco-Pereira, V. 2003, ApJ, 597, 970

Farmer, A. J., \& Goldreich, P. 2004, ApJ, 604, 671

Federer, W., Villinger, H., Howorka, F., et al. 1984, PhRvL, 52, 2084

Federman, S. R., Weber, J., \& Lambert, D. L. 1996, ApJ, 463, 181

Federrath, C. 2016, JPhCS, 719, 012002

Federrath, C., \& Klessen, R. S. 2013, ApJ, 763, 51

Felice, G. M., \& Kulsrud, R. M. 2001, ApJ, 553, 198

Fermi, E. 1949, PhRv, 75, 1169

Ferrière, K. M. 2001, RvMP, 73, 1031

Field, G. B., Goldsmith, D. W., \& Habing, H. J. 1969, ApJL, 155, L149

Fuente, A., \& Martín-Pintado, J. 1997, ApJL, 477, L107

Fujita, Y., Kimura, S., \& Ohira, Y. 2013, MNRAS, 432, 1434

Fujita, Y., \& Ohira, Y. 2011, ApJ, 738, 182

Gabici, S. 2011, in Cosmic Rays for Particle and Astroparticle Physics, ed. S. Giani, C. Leroy, \& P. G. Rancoita (Singapore: World Scientific), 343

Gabici, S., Aharonian, F. A., \& Casanova, S. 2009, MNRAS, 396, 1629

Gaches, B. A. L., Offner, S. S. R., \& Bisbas, T. G. 2019, ApJ, 878, 105

Gaggero, D. 2012, Cosmic Ray Diffusion in the Galaxy and Diffuse Gamma Emission, Springer Theses (Berlin: Springer)

Gaggero, D., Grasso, D., Marinelli, A., Taoso, M., \& Urbano, A. 2017, PhRvL, 119,031101

Gaia Collaboration, Brown, A. G. A., Vallenari, A., et al. 2018, A\&A, 616, A1

Galli, D., Walmsley, M., \& Gonçalves, J. 2002, A\&A, 394, 275

Gammie, C. F., \& Ostriker, E. C. 1996, ApJ, 466, 814

Gao, Y., Xu, H., \& Law, C. K. 2015, ApJ, 799, 227

Geballe, T. R., Indriolo, N., McCall, B. J., \& Oka, T. 2007, BAAS, 39, 985

Geballe, T. R., McCall, B. J., Hinkle, K. H., \& Oka, T. 1999, ApJ, 510, 251

Ginzburg, V. L., \& Syrovatskii, S. I. 1964, The Origin of Cosmic Rays (New York: Macmillan)

Girart, J. M., Rao, R., \& Marrone, D. P. 2006, Sci, 313, 812

Glassgold, A. E., \& Langer, W. D. 1974, ApJ, 193, 73

Goldsmith, P. F. 2001, ApJ, 557, 736

Goldsmith, P. F., \& Langer, W. D. 1978, ApJ, 222, 881

H.E.S.S. Collaboration, Abdalla, H., Abramowski, A., et al. 2018a, A\&A, 612, A1

H.E.S.S. Collaboration, Abdalla, H., Abramowski, A., et al. 2018b, A\&A, 612, A9

Hairer, E., Nørsett, S., \& Wanner, G. 1993, Solving Ordinary Differential Equations II: Stiff and Differential-Algebraic Problems (Berlin: Springer)

Harju, J., Juvela, M., Schlemmer, S., et al. 2008, A\&A, 482, 535

Harris, C. R., Millman, K. J., van der Walt, S. J., et al. 2020, Natur, 585, 357 Hartquist, T. W., Black, J. H., \& Dalgarno, A. 1978, MNRAS, 185, 643
Harvey, P. M., Huard, T. L., Jørgensen, J. K., et al. 2008, ApJ, 680, 495

Hasselmann, K., \& Wibberenz, G. 1968, ZGeo, 328, 269

Hayakawa, S., Nishimura, S., \& Takayanagi, T. 1961, PASJ, 13, 184

Hildebrand, R. H., Kirby, L., Dotson, J. L., Houde, M., \& Vaillancourt, J. E. 2009, ApJ, 696, 567

Hillas, A. M. 2006, arXiv:astro-ph/0607109

Hollenbach, D., Kaufman, M. J., Neufeld, D., Wolfire, M., \& Goicoechea, J. R. 2012, ApJ, 754, 105

Houde, M., Vaillancourt, J. E., Hildebrand, R. H., Chitsazzadeh, S., \& Kirby, L. 2009, ApJ, 706, 1504

Hull, C. L. H., Mocz, P., Burkhart, B., et al. 2017, ApJL, 842, L9

Indriolo, N. 2012, RSPTA, 370, 5142

Indriolo, N. 2013, in Cosmic Rays in Star-Forming Environments, Astrophysics and Space Science, Vol. 34, ed. D. F. Torres \& O. Reimer (Berlin: Springer), 83

Indriolo, N., Geballe, T. R., Oka, T., \& McCall, B. J. 2007, ApJ, 671, 1736

Indriolo, N., \& McCall, B. J. 2012, ApJ, 745, 91

Indriolo, N., Neufeld, D. A., Gerin, M., et al. 2015, ApJ, 800, 40

Ivlev, A. V., Dogiel, V. A., Chernyshov, D. O., et al. 2018, ApJ, 855, 23

Jaacks, J., Finkelstein, S. L., \& Bromm, V. 2019, MNRAS, 488, 2202

Jacob, S., \& Pfrommer, C. 2017, MNRAS, 467, 1449

Jenkins, E. B., Jura, M., \& Loewenstein, M. 1983, ApJ, 270, 88

Jokipii, J. R. 1966, ApJ, 146, 480

Jones, D. I. 2014, ApJL, 792, L14

Jones, J., Birkinshaw, K., \& Twiddy, N. 1981, CPL, 77, 484

Jura, M. 1975, ApJ, 197, 581

Juvela, M., \& Ysard, N. 2011, ApJ, 739, 63

Kafexhiu, E., Aharonian, F., Taylor, A. M., \& Vila, G. S. 2014, PhRvD, 90, 123014

Kalvāns, J. 2018, ApJS, 239, 6

Kirk, J. G., Schlickeiser, R., \& Schneider, P. 1988, ApJ, 328, 269

Klippenstein, S. J., Georgievskii, Y., \& McCall, B. J. 2010, JPCA, 114, 278

Ko, C.-M. 1992, A\&A, 259, 377

Koch, P. M., Tang, Y.-W., Ho, P. T. P., et al. 2014, ApJ, 797, 99

Kossmann, H., Schwarzkopf, O., \& Schmidt, V. 1990, JPhB, 23, 301

Kotera, K., \& Olinto, A. V. 2011, ARA\&A, 49, 119

Krause, J., Morlino, G., \& Gabici, S. 2015, arXiv:1507.05127

Krymskii, G. F. 1977, DoSSR, 234, 1306

Kudoh, T., \& Basu, S. 2008, ApJL, 679, L97

Kulsrud, R., \& Pearce, W. P. 1969, ApJ, 156, 445

Kulsrud, R. M. 2005, Plasma Physics for Astrophysics (Princeton, NJ: Princeton Univ. Press)

Kulsrud, R. M., \& Cesarsky, C. J. 1971, ApL, 8, 189

Lacki, B. C., \& Beck, R. 2013, MNRAS, 430, 3171

Lacki, B. C., Thompson, T. A., \& Quataert, E. 2010, ApJ, 717, 1

Lada, C. J., Alves, J., \& Lada, E. A. 1999, ApJ, 512, 250

Larson, R. L., Evans, N. J., II, Green, J. D., \& Yang, Y.-L 2015, ApJ, 806, 70

Latter, W. B., Walker, C. K., \& Maloney, P. R. 1993, ApJL, 419, L97

Lazarian, A., Goodman, A. A., \& Myers, P. C. 1997, ApJ, 490, 273

Lazarian, A., \& Hoang, T. 2007, MNRAS, 378, 910

Lazarian, A., \& Pogosyan, D. 2016, ApJ, 818, 178

Lee, J.-E., Evans, N. J., II, Shirley, Y. L., \& Tatematsu, K. 2003, ApJ, 583, 789

Lequeux, J. 2005, The Interstellar Medium

Li, H.-b., Dowell, C. D., Goodman, A., Hildebrand, R., \& Novak, G. 2009, ApJ, 704, 891

Li, H.-B., Yuen, K. H., Otto, F., et al. 2015, Natur, 520, 518

Lin, S.-J., Pagani, L., Lai, S.-P., Lefèvre, C., \& Lique, F. 2020, A\&A, 635, A188

Liu, X., \& Shemansky, D. E. 2004, ApJ, 614, 1132

Loewenstein, M., Zweibel, E. G., \& Begelman, M. C. 1991, ApJ, 377, 392

Mac Low, M.-M., \& Klessen, R. S. 2004, RvMP, 76, 125

Martin, C. E., Heyvaerts, J., \& Priest, E. R. 1997, A\&A, 326, 1176

Martínez-Gómez, D., Soler, R., \& Terradas, J. 2018, ApJ, 856, 16

McCall, B. J., Huneycutt, A. J., Saykally, R. J., et al. 2004, PhRvA, 70, 052716

McElroy, D., Walsh, C., Markwick, A. J., et al. 2013, A\&A, 550, A36

McKee, C. F., \& Zweibel, E. G. 1995, ApJ, 440, 686

Mestel, L. 1966, MNRAS, 133, 265

Mestel, L., \& Spitzer, L., Jr. 1956, MNRAS, 116, 503

Millar, T. J., Farquhar, P. R. A., \& Willacy, K. 1997, A\&AS, 121, 139

Minter, A. H., \& Spangler, S. R. 1996, ApJ, 458, 194

Mitchell, J. B. A. 1990, PhR, 186, 215

Morfill, G. E. 1982a, MNRAS, 198, 583

Morfill, G. E. 1982b, ApJ, 262, 749

Morlino, G., \& Gabici, S. 2015, MNRAS, 451, L100

Moskalenko, I. V., Strong, A. W., Ormes, J. F., \& Potgieter, M. S. 2002, ApJ, 565,280 
Mouschovias, T. C. 1991, in NATO Advanced Science Institutes (ASI) Series C, ed. C. J. Lada \& N. D. Kylafis, Vol. 342 (Berlin: Springer), 61

Mouschovias, T. C., \& Ciolek, G. E. 1999, in NATO Advanced Science Institutes (ASI) Series C, ed. C. J. Lada \& N. D. Kylafis, Vol. 540 (Berlin: Springer), 305

Murphy, R. J., Dermer, C. D., \& Ramaty, R. 1987, ApJS, 63, 721

Myers, P. 1995, in Molecular Clouds and Star Formation, ed. C. Yuan \& J.-H. You (Singapore: World Scientific)

Neufeld, D. A., \& Wolfire, M. G. 2017, ApJ, 845, 163

Neufeld, D. A., Goicoechea, J. R., Sonnentrucker, P., et al. 2010, A\&A, 521, L10

Osterbrock, D. E. 1989, Astrophysics of Gaseous Nebulae and Active Galactic Nuclei (Herndon, VA: University Science Books)

Owen, E. R., Jacobsen, I. B., Wu, K., \& Surajbali, P. 2018, MNRAS, 481, 666

Owen, E. R., Jin, X., Wu, K., \& Chan, S. 2019a, MNRAS, 484, 1645

Owen, E. R., Wu, K., Jin, X., Surajbali, P., \& Kataoka, N. 2019b, A\&A, 626, A85

Padoan, P., \& Nordlund, A. 1999, ApJ, 526, 279

Padoan, P., \& Scalo, J. 2005, ApJL, 624, L97

Padovani, M., \& Galli, D. 2011, A\&A, 530, A109

Padovani, M., \& Galli, D. 2018, A\&A, 620, L4

Padovani, M., Galli, D., \& Glassgold, A. E. 2009, A\&A, 501, 619

Padovani, M., Galli, D., Ivlev, A. V., Caselli, P., \& Ferrara, A. 2018, A\&A, 619, A144

Padovani, M., Hennebelle, P., \& Galli, D. 2013, A\&A, 560, A114

Padovani, M., Hennebelle, P., Marcowith, A., \& Ferrière, K. 2015, A\&A, 582, L13

Padovani, M., Marcowith, A., Hennebelle, P., \& Ferrière, K. 2016, A\&A, 590, A8

Padovani, M., Ivlev, A. V., Galli, D., et al. 2020, SSRv, 216, 29

Pagani, L., Bacmann, A., Cabrit, S., \& Vastel, C. 2007, A\&A, 467, 179

Pagani, L., Lefèvre, C., Juvela, M., Pelkonen, V. M., \& Schuller, F. 2015, A\&A, 574, L5

Pagani, L., Vastel, C., Hugo, E., et al. 2009, A\&A, 494, 623

Pan, L., \& Padoan, P. 2009, ApJ, 692, 594

Patrignani, C., et al. 2016, ChPhC, 40, 100001

Percival, D. B., \& Walden, A. T. 1993, Spectral Analysis for Physical Applications: Multitaper and Conventional Univariate Techniques (Cambridge: Cambridge Univ. Press)

Phan, V. H. M., Morlino, G., \& Gabici, S. 2018, MNRAS, 480, 5167

Planck Collaboration, Ade, P. A. R., Aghanim, N., et al. 2016, A\&A, 586, A138

Prasad, S. S., \& Huntress, W. T., Jr. 1980, ApJS, 43, 1

Press, W., Teukolsky, S., Vetterling, W., \& Flannery, B. 2007, Numerical Recipes 3rd Edition: The Art of Scientific Computing (Cambridge: Cambridge Univ. Press)

Press, W. H., Teukolsky, S. A., Vetterling, W. T., \& Flannery, B. P. 1992, Numerical Recipes in FORTRAN. The Art of Scientific Computing (Cambridge: Cambridge Univ. Press)

Price, D. J., \& Bate, M. R. 2008, MNRAS, 385, 1820

Protheroe, R. J., Ott, J., Ekers, R. D., Jones, D. I., \& Crocker, R. M. 2008 , MNRAS, 390, 683

Rao, R., Girart, J. M., Marrone, D. P., Lai, S.-P., \& Schnee, S. 2009, ApJ, 707, 921

Redaelli, E., Alves, F. O., Santos, F. P., \& Caselli, P. 2019, A\&A, 631, A154

Rodríguez, L. F. R. 2005, in ASP Conf. Ser. 344, The Cool Universe: Observing Cosmic Dawn, ed. C. Lidman \& D. Alloin (San Francisco, CA: ASP), 146

Rudd, M. E. 1991, PhRvA, 44, 1644

Rudd, M. E., Goffe, T. V., Dubois, R. D., Toburen, L. H., \& Ratcliffe, C. A. 1983, PhRvA, 28, 3244

Rudd, M. E., Kim, Y. K., Madison, D. H., \& Gallagher, J. W. 1985, RvMP, 57,965
Ruszkowski, M., Yang, H.-Y. K., \& Reynolds, C. S. 2017, ApJ, 844, 13

Salem, M., Bryan, G. L., \& Corlies, L. 2016, MNRAS, 456, 582

Schlickeiser, R. 2002, Cosmic Ray Astrophysics

Schlickeiser, R., \& Achatz, U. 1993a, JPIPh, 49, 63

Schlickeiser, R., \& Achatz, U. 1993b, JPIPh, 50, 85

Schulz-Dubois, E. O., \& Rehberg, I. 1981, ApPhy, 24, 323

Seifried, D., \& Walch, S. 2015, MNRAS, 452, 2410

Sikora, M., Kirk, J. G., Begelman, M. C., \& Schneider, P. 1987, ApJL, 320, L81

Silsbee, K., \& Ivlev, A. V. 2019, ApJ, 879, 14

Silsbee, K., Ivlev, A. V., Padovani, M., \& Caselli, P. 2018, ApJ, 863, 188

Skorodko, T., Bashkanov, M., Bogoslawsky, D., et al. 2008, EPJA, 35, 317

Skrutskie, M. F., Cutri, R. M., Stiening, R., et al. 2006, AJ, 131, 1163

Sofia, U. J., Lauroesch, J. T., Meyer, D. M., \& Cartledge, S. I. B. 2004, ApJ, 605,272

Sonnentrucker, P., Welty, D. E., Thorburn, J. A., \& York, D. G. 2007, ApJS, 168,58

Spitzer, L., Jr., \& Tomasko, M. G. 1968, ApJ, 152, 971

Stancil, P. C., Schultz, D. R., Kimura, M., et al. 1999, A\&AS, 140, 225

Straub, H. C., Renault, P., Lindsay, B. G., Smith, K. A., \& Stebbings, R. F. 1996, PhRvA, 54, 2146

Strong, A. W., Dickinson, C., \& Murphy, E. J. 2014, arXiv:1412.4500

Strong, A. W., Moskalenko, I. V., \& Ptuskin, V. S. 2007, ARNPS, 57, 285

Strong, A. W., Moskalenko, I. V., \& Reimer, O. 2000, ApJ, 537, 763

Tang, Y.-W., Ho, P. T. P., Koch, P. M., et al. 2009, ApJ, 700, 251

Tang, Y.-W., Koch, P. M., Peretto, N., et al. 2019, ApJ, 878, 10

Theard, L. P., \& Huntress, W. T. 1974, JChPh, 60, 2840

Thompson, K. L., Troland, T. H., \& Heiles, C. 2019, ApJ, 884, 49

Thompson, T. A., Quataert, E., \& Waxman, E. 2007, ApJ, 654, 219

Torres, D. F. 2004, ApJ, 617, 966

van der Tak, F. F. S., \& van Dishoeck, E. F. 2000, A\&A, 358, L79

van Dishoeck, E. F., \& Black, J. H. 1986, ApJS, 62, 109

Vázquez-Semadeni, E., Banerjee, R., Gómez, G. C., et al. 2011, MNRAS, 414, 2511

Virtanen, P., Gommers, R., Oliphant, T. E., et al. 2020, Nature Methods, 17,261

Walker, M. A. 2016, ApJ, 818, 23

Wang, J.-W., Lai, S.-P., Clemens, D. P., et al. 2020, ApJ, 888, 13

Wang, J.-W., Lai, S.-P., Eswaraiah, C., et al. 2017, ApJ, 849, 157

Wang, J.-W., Lai, S.-P., Eswaraiah, C., et al. 2019, ApJ, 876, 42

Webber, W. R. 1998, ApJ, 506, 329

Wentzel, D. G. 1969, ApJ, 156, 303

Wentzel, D. G. 1971, ApJ, 163, 503

Wentzel, D. G. 1974, ARA\&A, 12, 71

Whittet, D. C. B., Hough, J. H., Lazarian, A., \& Hoang, T. 2008, ApJ, 674, 304

Whitworth, A. P., \& Jaffa, S. E. 2018, A\&A, 611, A20

Whitworth, A. P., \& Ward-Thompson, D. 2001, ApJ, 547, 317

Wiener, J., Oh, S. P., \& Guo, F. 2013a, MNRAS, 434, 2209

Wiener, J., Zweibel, E. G., \& Oh, S. P. 2013b, ApJ, 767, 87

Wiener, N. 1930, AcMa, 55, 117

Xu, S., \& Zhang, B. 2016, ApJ, 824, 113

Yamamoto, S. 2017, Introduction to Astrochemistry: Chemical Evolution from Interstellar Clouds to Star and Planet Formation (Tokyo: Springer Japan)

Yoast-Hull, T. M., Gallagher, J. S., \& Zweibel, E. G. 2015, MNRAS, 453, 222

Yoast-Hull, T. M., Gallagher, J. S., \& Zweibel, E. G. 2016, MNRAS, 457, L29

Yusef-Zadeh, F., Hewitt, J. W., Wardle, M., et al. 2013, ApJ, 762, 33

Yusef-Zadeh, F., Law, C., \& Wardle, M. 2002, ApJL, 568, L121

Zhang, C.-P., \& Li, G.-X. 2017, MNRAS, 469, 2286

Zhang, Q., Qiu, K., Girart, J. M., et al. 2014, ApJ, 792, 116

Zhang, Y., Guo, Z., Wang, H. H., \& Li, H. b. 2019, ApJ, 871, 98

Zweibel, E. G., \& Shull, J. M. 1982, ApJ, 259, 859 